\title{
NONCOMMUTATIVE BLOWUPS OF ELLIPTIC ALGEBRAS
}

\author{
D. ROGALSKI, S. J. SIERRA, AND J. T. STAFFORD
}

\begin{abstract}
We develop a ring-theoretic approach for blowing up many noncommutative projective surfaces. Let $T$ be an elliptic algebra (meaning that, for some central element $g \in T_{1}, T / g T$ is a twisted homogeneous coordinate ring of an elliptic curve $E$ at an infinite order automorphism). Given an effective divisor $\mathbf{d}$ on $E$ whose degree is not too big, we construct a blowup $T(\mathbf{d})$ of $T$ at $\mathbf{d}$ and show that it is also an elliptic algebra. Consequently it has many good properties: for example, it is strongly noetherian, Auslander-Gorenstein, and has a balanced dualizing complex. We also show that the ideal structure of $T(\mathbf{d})$ is quite rigid. Our results generalise those of the first author in $[\mathrm{Ro}$.

In the companion paper [RSS, we apply our results to classify orders in (a Veronese subalgebra of) a generic cubic or quadratic Sklyanin algebra.
\end{abstract}

\section{Contents}

1. Introduction

2. Basic properties

3. A categorical equivalence for right ideals

4. Certain right ideals of $T$ and their Hilbert series

5. The blowups $T(\mathbf{d})$

6. Relating left and right ideals

7. Divisors and exceptional line modules

8. Minimal sporadic ideals

9. On the nonexistence of sporadic ideals

Index of Notation

References

2000 Mathematics Subject Classification. 14A22, 14H52, 16E65, 16P40, 16S38, 16W50, $18 \mathrm{E} 15$.

Key words and phrases. Noncommutative projective geometry, noncommutative surfaces, Sklyanin algebras, noetherian graded rings, noncommutative blowing up.

The first author is partially supported by NSF grants DMS-0900981 and DMS-1201572.

The second author was partially supported by an NSF Postdoctoral Research Fellowship, grant DMS-0802935.

The third author is a Royal Society Wolfson Research Merit Award Holder. 


\section{INTRODUCTION}

Blowing up a projective surface at a point, or more formally applying a monoidal transformation, is a fundamental operation in commutative algebraic geometry. The aim of this paper is to study noncommutative analogues of this concept.

There are two basic approaches to noncommutative projective algebraic geometry: one may study graded rings which have some of the properties of homogeneous coordinate rings of projective varieties, or one may study categories which are similar to the category of coherent sheaves on a projective variety. Here we take the first approach. We show, in a unified and ring-theoretic way, that there is a noncommutative analogue of the blowing-up process, and we establish many of the properties of the resulting algebras. Our results are applied in the companion paper [RSS] to classify orders in (Veronese subalgebras of) Sklyanin algebras.

Fix an algebraically closed ground field $\mathbb{k}$ and let $T=\mathbb{k} \oplus T_{1} \oplus T_{2} \oplus \ldots$ be a connected graded $\mathbb{k}$-algebra that is a domain. We say that $T$ is an elliptic algebra if there exists a central element $g \in T_{1}$ so that $T / g T$ is a twisted homogeneous coordinate ring $B(E, \mathcal{M}, \tau)$ on an elliptic curve $E$, where $\mathcal{M}$ is ample and $\tau \in \operatorname{Aut}(E)$ has infinite order. (See Section 2 for background material on these coordinate rings.) Elliptic algebras are noncommutative analogues of anticanonical coordinate rings of projective (Fano or del Pezzo) surfaces; the best-known examples are Veronese subalgebras of generic Sklyanin algebras. As we will see in a moment, they are extremely well-behaved.

Let $T$ be an elliptic algebra. We define the degree of $T$ to be $\mu=\operatorname{deg} \mathcal{M}=\operatorname{dim} T_{1}-1$. Our main result is that we can use the points of $E$ to "blow up" $T$ and create further elliptic algebras. More specifically, let $\mathbf{d}$ be an effective divisor on $E$, with $\operatorname{deg} \mathbf{d}<\mu$. We define a graded subalgebra $T(\mathbf{d})$ of $T$, and show that $T(\mathbf{d})$ has the properties described in the following theorem. Here, if $R$ is a noetherian graded domain its graded quotient ring $Q_{g r}(R)$ is formed by inverting all nonzero homogeneous elements ( $\mathrm{MR}$, Corollary 8.1.21] and [NV, C.I.1.6]). The ring $R$ is a maximal order if there exists no order $R \varsubsetneqq R^{\prime} \subseteq Q_{g r}(R)$ with $a R^{\prime} b \subseteq R$ for some nonzero $a, b \in R$. Undefined terms in the theorem can be found in Section 2 or in [Ro].

Theorem 1.1. (Theorem 5.3) Let $T, \mu, \mathbf{d}$ be as above, and write $d=\operatorname{deg} \mathbf{d}$. Set $R=T(\mathbf{d})$. Then:

(1) $R$ is an elliptic algebra of degree $\mu-d$, with $R / g R \cong B(E, \mathcal{M}(-\mathbf{d}), \tau)$. As such, $R$ is strongly noetherian, Auslander-Gorenstein, Cohen-Macaulay, and a maximal order. In addition, $R$ satisfies the Artin-Zhang $\chi$ conditions and possesses a balanced dualizing complex.

(2) $R$ has Hilbert series $h_{R}(t)=\sum\left(\operatorname{dim}_{\mathbb{k}} R_{i}\right) t^{i}=h_{T}(t)-d /(1-t)^{3}$, and $Q_{g r}(R)=Q_{g r}(T)$.

(3) $R(\mathbf{d})$ is generated in degree 1 if $\mu-d \geq 2$. If $\mu-d=1$, then $R$ is generated in degrees $\leq 2$, and $R^{(2)}$ is generated in degree 1.

(4) (Proposition 5.4) Further, T(d) can be obtained by iterating a series of one-point blowups.

For reasons why it is appropriate to regard $T(\mathbf{d})$ as a blowup, see Section 5 . In particular various basic properties of a commutative blowup $\pi: \widetilde{X} \rightarrow X$ do have noncommutative analogues. For one-point blowups, we prove: 
Proposition 1.2. (Proposition 7.13) Let $T$ be as above, with $\operatorname{deg} T=\mu>1$. Let $p \in E$ and set $R=T(p)$. Then there is a finitely generated right $R$-module $L$ so that $T / R \cong \bigoplus_{i \geq 1} L[-i]$ as right $R$-modules. Further, $L$ is $g$-torsionfree in the obvious sense, and has Hilbert series $h_{L}(t)=1 /(1-t)^{2}$.

Remark 1.3. It is natural to consider $L$ as the exceptional line for the noncommutative blowup $T(p)$ of $T$.

The elliptic algebras that we are most interested in are blowups of Veronese subalgebras of generic Sklyanin algebras. We show that these particular elliptic algebras have a relatively rigid ideal structure.

Theorem 1.4. (Theorem 8.8) Let $S$ be a generic cubic or quadratic Sklyanin algebra, with central element $g \in S_{\gamma}$. Let $T=S^{(\gamma)}$ and $\mu=\operatorname{deg} T$. Let $\mathbf{d}$ be an effective divisor on $E$ with $\operatorname{deg} \mathbf{d}<\mu$. Then $T(\mathbf{d})$ has a minimal sporadic ideal $I$ in the following sense: $T / I$ has Gelfand-Kirillov dimension $\leq 1$, and for any graded ideal $J \subseteq T$ with $\mathrm{GKdim} T / J=1$, we have $J_{n} \supseteq I_{n}$ for $n \gg 0$.

The above results generalise those of the first author in $[\mathrm{Ro}$. In that paper, Theorems 1.1 and 1.4 are proved where $T=S^{(3)}$ is the 3 rd Veronese of a generic quadratic Sklyanin algebra, and $\operatorname{deg} \mathbf{d} \leq 7=\mu-2$. Allowing deg $\mathbf{d}=\mu-1$ requires one to overcome technical difficulties, as the resulting rings are no longer generated in degree 1. The rings $T(\mathbf{d})$ also appear in Van den Bergh's memoir [VB2]. Van den Bergh, however, works mostly on the categorical level; in contrast, our approach is more elementary and is more amenable to computation.

The main motivation of our work is to be able to answer the following question:

Question 1.5. ( $[\mathrm{Ro}$, Question 1.3]) Can one classify all maximal orders $R$ inside a generic (quadratic) Sklyanin algebra $S$ with $Q_{g r}(R)=Q_{g r}\left(S^{(n)}\right)$ for some $n$ ?

(We remark that maximal orders, the noncommutative analogues of integrally closed rings, are a natural class to consider in such a classification problem.) In the companion paper [RSS, we solve Question 1.5] for $n \in 3 \mathbb{N}$. In particular, we prove:

Theorem 1.6. ([ $\mathrm{RSS}$, Theorem 1.2, Corollary 1.4, and Theorem 5.25]) Let $T=S^{(3)}$ be as above, and let $R$ be a maximal order in $T$ such that $R \nsubseteq \mathbb{k}+g T$. Then $R$ is noetherian and is obtained from $T$ by the analogue of a blowup of $T$ at a possibly non-effective divisor $\mathbf{x}$. Further, $R$ is equivalent to some $T(\mathbf{d})$, where $\mathbf{d}$ is effective with $\operatorname{deg} \mathbf{d} \leq 8=\mu-1$.

In [RSS, we also obtain a classification (up to Veronese subalgebras) of arbitrary orders in $S^{(3 n)}$.

One of the authors' ultimate goals is to classify all algebras with the same graded quotient ring as a Sklyanin algebra. Such a result would contribute significantly to solving the fundamental problem of the classification of noncommutative projective surfaces $\underline{\mathrm{Ar}}$. From a ring-theoretic perspective, one can frame the problem as: what are the graded domains with GK-dimension 3?

Let $R$ be such an algebra. Then $Q_{g r}(R) \cong D\left[z, z^{-1} ; \tau\right]$ for some division ring $D$, called the function skewfield of $R$, and some $\tau \in \operatorname{Aut}_{\mathbb{k}}(D)$. The study of birationally equivalent, irreducible projective varieties 
can be interpreted ring-theoretically as the study of different graded domains $R$ with the same function skewfield. As Artin has argued in $[\mathrm{Ar}$, one hopes that algebras (or at least maximal orders) in the same birational class are related through some sort of blowing up and blowing down processes. By Theorem 1.6, this is true for orders in $T$.

Organisation of the paper. In outline, Section 2 contains general background material and basic properties of elliptic algebras while Section 3 reviews the necessary technical machinery from [VB2]. This leads directly in Section 4 to the construction of the blowups $T(\mathbf{d})$. We prove Theorem 1.1 and other basic properties of these rings in Section 5 . Section 6 contains results relating left and right ideals of $T(\mathbf{d})$. In Section 7 we construct the exceptional line module of a one-point blowup. In Section 8 we study the ideal theory of $T(\mathbf{d})$ and prove Proposition 1.2 and Theorem 1.4. Finally, in Section 9 we determine circumstances when a blowup $T(\mathbf{d})$ has no nontrivial sporadic ideals. At the end of the paper we provide an index of notation.

Acknowledgements. Part of this material is based upon work supported by the National Science Foundation under Grant No. 0932078 000, while the authors were in residence at the Mathematical Science Research Institute (MSRI) in Berkeley, California, during the Spring semester of 2013. During this trip, Sierra was also partially supported by the Edinburgh Research Partnership in Engineering and Mathematics, and Stafford was partially supported by the Clay Mathematics Institute and Simons Foundation. The authors gratefully acknowledge the support of all these organisations.

\section{BASIC PROPERTIES}

We begin by reviewing some definitions and basic background material for the paper.

Throughout, fix an algebraically closed base field $\mathbb{k}$. Let $E$ be a projective $\mathbb{k}$-scheme (which will always be a (necessarily smooth) elliptic curve), with an invertible sheaf $\mathcal{L}$ and an automorphism $\tau$. For any sheaf $\mathcal{F}$ on $E$ we write $\mathcal{F}^{\tau}=\tau^{*}(\mathcal{F})$ for the pullback along $\tau$. Using this data we can define a $T C R$ or twisted homogeneous coordinate ring $B(E, \mathcal{L}, \tau)=\bigoplus_{n \geq 0} H^{0}\left(E, \mathcal{L}_{n}\right)$. Here, $\mathcal{L}_{n}=\mathcal{L} \otimes \mathcal{L}^{\tau} \otimes \cdots \otimes \mathcal{L}^{\tau^{n-1}}$ and multiplication is defined by $f \star g=f \otimes\left(\tau^{m}\right)^{*}(g)$ for $f \in B_{m}$ and $g \in B_{n}$. We will typically use bold notation $\mathbf{d}$ for divisors on $E$ and write $\mathbf{d}^{\tau}=\tau^{-1}(\mathbf{d})$, so that, if $\mathcal{L} \cong \mathcal{O}_{E}(\mathbf{d})$, then $\mathcal{L}^{\tau}=\mathcal{O}_{E}\left(\mathbf{d}^{\tau}\right)$. Similarly, we write $\mathbf{d}_{n}=\mathbf{d}+\mathbf{d}^{\tau}+\cdots+\mathbf{d}^{\tau^{n-1}}$. Some of the special features of $B(E, \mathcal{L}, \tau)$ are described in [Ro, Section 3].

In this paper we always assume that $|\tau|=\infty$, which has the following useful consequences.

Lemma 2.1. ([Ro, Lemma 3.1]) Let $E$ be an elliptic curve with an automorphism $\tau$ of infinite order.

(1) Given invertible sheaves $\mathcal{L}, \mathcal{M}$ on $E$, the natural map

$$
H^{0}(E, \mathcal{L}) \otimes H^{0}(E, \mathcal{M}) \rightarrow H^{0}(E, \mathcal{L} \otimes \mathcal{M})
$$

is surjective if $\operatorname{deg} \mathcal{L} \geq 2$ and $\operatorname{deg} \mathcal{M} \geq 2$, except in the case when $\operatorname{deg} \mathcal{L}=2$ and $\mathcal{L} \cong \mathcal{M}$.

(2) If $\operatorname{deg} \mathcal{L} \geq 2$ then $B(E, \mathcal{L}, \tau)$ is generated in degree 1 . 
In this paper we will consider the following special class of rings. Here, an $\mathbb{N}$-graded $\mathbb{k}$-algebra $R$ is connected graded (cg) if $R_{0}=\mathbb{k}$ and $\operatorname{dim}_{\mathbb{k}} R_{n}<\infty$ for all $n$.

Hypothesis 2.2. Let $T$ be a $\mathrm{cg} \mathbb{k}$-algebra which is a domain with a central element $g \in T_{1}$ such that $T / g T \cong B=B(E, \mathcal{M}, \tau)$ for an elliptic curve $E$, invertible sheaf $\mathcal{M}$ of degree $\mu \geq 2$, and infinite order automorphism $\tau$. By [RSS, Lemma 2.7] (or by Lemma 2.1] and induction), $T$ is finitely generated in degree 1.

Given $X \subseteq T$, we always write $\bar{X}=(X+g T) / g T \subseteq B(E, \mathcal{M}, \tau)$.

One of the main ways of producing such a ring $T$ is to start with either the quadratic Sklyanin algebra or the cubic Sklyanin algebra $S$ of dimension 3. This has a central element $g \in S_{\gamma}$ (for $\gamma=3$, respectively 4) such that $S / g S \cong B(E, \mathcal{L}, \sigma)$. We always assume that $|\sigma|=\infty$, in which case, by a slight abuse of notation, we call $S$ a generic Sklyanin algebra. Then the Veronese $\operatorname{ring} T=S^{(\gamma)}$ satisfies Hypothesis 2.2 The hypothesis also holds for Veronese rings of generic Stephenson's algebras [St]. See [RSS, Examples 2.2] for the explicit definitions of these algebras.

The rings $T$ in Hypothesis 2.2 automatically have many good properties, as we next show. This requires the following definitions. The remaining terms in the proposition are not defined since they do not play a significant rôle in this paper; the relevant definitions can be found, for example, in [Ro, Section 2].

Definition 2.3. A ring $A$ is called Auslander-Gorenstein if it has finite injective dimension and satisfies the Gorenstein condition: if $p<q$ are non-negative integers and $M$ is a finitely generated $A$-module, then $\operatorname{Ext}_{A}^{p}(N, A)=0$ for every submodule $N$ of $\operatorname{Ext}_{A}^{q}(M, A)$. Set $j(M)=\min \left\{r \mid \operatorname{Ext}_{A}^{r}(M, A) \neq 0\right\}$ for the homological grade of $M$. An Auslander-Gorenstein ring $A$ of finite GK-dimension is called Cohen-Macaulay (or $\mathrm{CM}$ ), provided that $j(M)+\operatorname{GKdim}(M)=\operatorname{GKdim}(A)$ holds for every finitely generated $A$-module $M$. Finally, an $A$-module $M$ is called Cohen-Macaulay (or CM) if $\operatorname{Ext}_{A}^{i}(M, A)=0$ for all $i \neq j(M)$.

Proposition 2.4. Let $S$ be a $\mathrm{cg} \mathbb{k}$-algebra which is a domain with a central element $g \in S_{\gamma}$ for some $\gamma \geq 1$, such that $S / g S \cong B(E, \mathcal{L}, \sigma)$ for some elliptic curve $E$, invertible sheaf $\mathcal{L}$ with $\operatorname{deg} \mathcal{L} \geq 1$, and infinite order automorphism $\sigma$.

Then $S$ is strongly noetherian, Auslander-Gorenstein, $C M$ and a maximal order. Also, $S$ satisfies the Artin-Zhang $\chi$ conditions, has cohomological dimension 2 and possesses a balanced dualizing complex.

Proof. Use the proofs of [Ro, Theorems 6.3 and 6.7] (the hypothesis assumed there that $\operatorname{deg} \mathcal{L} \geq 2$ is not needed in the proofs). The one exception to this assertion is the fact that $S$ has a balanced dualizing complex, which follows from [VB1, Theorem 6.3].

Next, we review some of the categories used in the paper. Let $R$ be a cg noetherian $\mathbb{k}$-algebra and write Gr- $R$ for the category of $\mathbb{Z}$-graded right $R$-modules, with $\operatorname{Hom}_{\mathrm{Gr}-R}(-,-)$ being graded homomorphisms of degree zero. Let Tors- $R \subseteq$ Gr- $R$ be the full subcategory of torsion modules; thus modules $M$ with the property that for every $m \in M, m A_{\geq n}=0$ for some $n \geq 1$. Write gr- $R$ for the full subcategory of Gr- $R$ of finitely generated $R$-modules, with torsion subcategory tors- $R$. The quotient category qgr- $R=\operatorname{gr}-R /$ tors- $R$ has the 
same objects as gr- $R$, but morphisms are given by $\operatorname{Hom}_{\mathrm{qgr}-R}(\pi(M), \pi(N))=\lim _{n \rightarrow \infty} \operatorname{Hom}_{\mathrm{gr}-R}\left(M_{\geq n}, N\right)$, for $M, N \in \operatorname{gr}-R$. The quotient category $Y=\operatorname{Qgr}-R=\operatorname{Gr}-R /$ Tors- $R$ is defined similarly, and qgr- $R$ may be identified with the subcategory of noetherian objects in $Y$; see [AZ1, p. 234-5]. The quotient functor $\pi$ : $\operatorname{Gr}-R \rightarrow Y$ has a right adjoint, the section functor $\omega: Y \rightarrow \operatorname{Gr}-R$ given by $\omega(\mathcal{F})=\bigoplus_{n \in \mathbb{Z}} \operatorname{Hom}_{Y}\left(\mathcal{O}_{Y}, \mathcal{F}[n]\right)$. Here $\mathcal{O}_{Y}=\pi(R)$ and $[n]$ is the shift functor on $Y$, induced by the shift of graded modules $M \mapsto M[n]$ where $M[n]_{m}=M_{n+m}$.

If $J$ is a graded right ideal of $R$, write $J^{\text {sat }}=\left\{x \in R \mid x R_{\geq n} \subseteq J\right.$ for some $\left.n \geq 0\right\}$ for the saturation of $J$. It is the unique largest right ideal $K \supseteq J$ such that $\operatorname{dim}_{\mathbb{k}}(K / J)<\infty$. We call $J$ saturated if $J=J^{\text {sat }}$.

Lemma 2.5. Assume that $T$ satisfies Hypothesis 2.2.

(1) The natural map $T \rightarrow \omega(\pi(T))$ is an isomorphism of rings.

(2) Given any right ideal $J$ of $T$, we have $\omega(\pi(J))=J^{\text {sat }} \subseteq T$.

Proof. (1) By [AZ1, (3.12.3)], for any $M \in$ gr- $T$ there is an exact sequence

$$
0 \rightarrow t(M) \rightarrow M \rightarrow \omega \pi(M) \rightarrow \lim _{n \rightarrow \infty} \operatorname{Ext}^{1}\left(T / T_{\geq n}, M\right) \rightarrow 0,
$$

where $t(M)$ is the largest finite-dimensional submodule of $M$. Apply this equation with $M=T$. Then $t(T)=0$ since $T$ is a domain. By Proposition 2.4 and [RSS, Lemma 4.11(2)], $T$ has no non-trivial extensions by finite-dimensional modules and so $\lim _{n \rightarrow \infty} \operatorname{Ext}^{1}\left(T / T_{\geq n}, T\right)=0$. Thus the natural map $T \rightarrow \omega \pi(T)$ is an isomorphism. As in [AZ1, Section 4], it is also a ring homomorphism.

(2) Since $\omega$ is left exact, $J^{\prime}=\omega(\pi(J)) \subseteq T$ is a right ideal of $T$ and $J^{\prime} / J$ is a torsion module by applying the exact sequence to $M=J$. It is easy to see that $\omega(\pi(J))$ is already saturated, so that $J^{\prime}=J^{\text {sat }}$.

The following definitions will be used frequently.

Definition 2.6. Let $S$ be a cg $\mathbb{k}$-algebra with homogeneous central element $g \in S$. Let $M$ be a (right) $S$-module. The $g$-torsion submodule of $M$ is $\operatorname{tors}_{g}(M)=\left\{m \in M \mid m g^{n}=0\right.$ for some $\left.n \geq 1\right\}$. The module $M$ is $g$-torsion if $\operatorname{tors}_{g}(M)=M$ and $g$-torsionfree if $\operatorname{tors}_{g}(M)=0$. A graded vector subspace $V$ of $S$ is $g$-divisible if $V \cap S g=V g$; if $V$ is a right ideal, this is equivalent to $\operatorname{tors}_{g}(S / V)=0$.

Definition 2.7. We say that $M \in \operatorname{gr}-R$ is a point module if it has Hilbert series $h_{M}(t)=1 /(1-t)$. A shifted point module is a module of the form $M[n]$ for some integer $n$ and point module $M$. We remark that point modules are traditionally assumed to be cyclic, necessarily generated in degree 0 . However, that assumption is not convenient for us, since it is really only appropriate for algebras generated in degree one.

Gelfand-Kirillov (GK) dimension will be the main dimension function in this paper. Recall that $M \in$ $\operatorname{gr}-R$ is $\alpha$-pure if $\operatorname{GKdim}(N)=\alpha$ for all nonzero submodules $N \subseteq M$, and $\alpha$-critical if $\operatorname{GKdim}(M / N)<$ $\operatorname{GKdim}(M)$ for all such $N$. The next lemma clarifies the relationship between 1-critical modules and point modules over $B(E, \mathcal{L}, \sigma)$. Here, we write $\mathcal{I}_{p}$ for the ideal sheaf defining a closed point $p \in E$, so that $\mathcal{O}_{E} / \mathcal{I}_{p}=\mathbb{k}(p)$, the corresponding skyscraper sheaf. Since qgr- $B \sim \operatorname{coh} E$, the category of coherent sheaves on 
$E\left(\right.$ see $\left[\mathrm{AV}\right.$ Theorem 1.3]), there is also a corresponding simple object $\mathcal{O}_{p} \in$ qgr- $B$. Explicitly, $\mathcal{O}_{p}=\pi(P(p))$, where $P(p)=\bigoplus_{n \geq 0} H^{0}\left(E, \mathbb{k}(p) \otimes \mathcal{L}_{n}\right)$ is the right point module of $B$ corresponding to $p$.

Lemma 2.8. Let $B=B(E, \mathcal{L}, \sigma)$, where $E$ is elliptic, $\operatorname{deg} \mathcal{L} \geq 1$, and $\sigma$ has infinite order.

(1) If $M \in \operatorname{gr}-B$ is a point module, then $M_{\geq n}$ is 1 -critical for some $n \geq 0$.

(2) For any $p \in E, P(p)$ is a (not necessarily cyclic) point module for $B$. If $M \in \operatorname{gr}-B$ is 1 -critical, then $\pi(M)=\mathcal{O}_{p}$ in qgr- $B$ for some $p \in E$.

(3) If $\operatorname{deg} \mathcal{L} \geq 2$, then $M \in \operatorname{gr}-B$ is 1-critical if and only if it is a shifted cyclic point module.

Proof. (1) Since $\operatorname{GKdim}(M)=1$ and $M$ has a unique largest finite dimensional submodule, $M_{\geq n}$ is 1-pure for $n \gg 0$. As such, $M_{\geq n}$ has a cyclic 1-critical submodule, say $Q$ (use the proof of [MR, Proposition 6.2.20]). But $\operatorname{dim}_{\mathbb{k}} Q_{m}=1$ for all $m \gg 0$ by [RSS, Corollary 3.7(2)]. So $M_{\geq m}=Q_{\geq m}$ is 1-critical for all such $m$.

(2) This follows from the equivalence of categories $\operatorname{coh} E \rightarrow \operatorname{qgr}-B$ and the fact that $\pi(M)$ is a simple object in qgr- $B$ (see [RSS, Corollary 3.7(1)] and its proof).

(3) This is standard. In more detail, assume that $M$ is 1-critical. By [RSS, Corollary 3.7(2)], $\operatorname{dim}_{\mathrm{k}} M_{n} \leq 1$ for all $n \in \mathbb{Z}$, with equality for $n \gg 0$. As $B$ is now generated in degree 1 (see Lemma 2.1) and $M$ is 1-pure, this means there is $j \in \mathbb{Z}$ such that $\operatorname{dim}_{\mathbb{k}} M_{n}=1$ for $n \geq j$ and $\operatorname{dim}_{\mathbb{k}} M_{n}=0$ for $n<j$. The module $M$ is then forced to be cyclic, generated in degree $j$, and so $M$ is a shifted cyclic point module.

\section{A CATEgorical EQUivalence FOR RIght idEALS}

For the whole of this section, fix a ring $T$ satisfying Hypothesis 2.2 and its associated notation, in particular $E$ is an elliptic curve with an infinite order automorphism $\tau$.

The main goal of this section is to review a categorical equivalence developed by Van den Bergh and Van Gastel [VV], which gives a formally useful way to describe and work with cyclic T-modules of GKdimension 1 and their defining ideals. We then use this to associate certain important right $T$-ideals to lists of divisors on $E$, and begin to analyse their properties.

Let $X=$ qgr- $T$ and $Y=$ qgr- $B$, for $B=T / g T$. Then $Y$ is a subcategory of $X$ with $\operatorname{coh} E \sim Y$ via the map $\mathcal{F} \mapsto \pi\left(\bigoplus_{n \geq 0} H^{0}\left(E, \mathcal{F} \otimes \mathcal{M}_{n}\right)\right)$ (see [AV]). Thus, we have the main hypothesis of [VB2, Chapter 5]; namely, in the language of that paper, $Y$ is a commutative $\mathrm{CM}$ curve embedded as a divisor in the noetherian quasi-scheme $X$. Moreover, [VB2], Hypothesis $(*)$ (which demands that objects in qgr- $B$ have finite injective dimension in qgr- $T$ ) holds automatically; see [VB2, Hypothesis $\left(*^{\prime}\right)$ and the discussion there.

One would like to understand the category $\mathcal{C}_{f}$ of finite length objects in $X$ whose composition factors lie in $Y$. Following [VB2, Section 5], we let $\mathcal{C}_{f, p}$ be the subcategory of $X$ consisting of finite-length objects whose Jordan-Holder quotients are all simple objects of the form $\mathcal{O}_{\tau^{i}(p)}$ for various $i \in \mathbb{Z}$. One may show that $\operatorname{dim}_{\mathbb{k}} \operatorname{Ext}_{\text {qgr- } B}^{1}\left(\mathcal{O}_{p}, \mathcal{O}_{q}\right)=\delta_{p, q}$, but $\operatorname{dim}_{\mathbb{k}} \operatorname{Ext}_{\text {qgr-T }}^{1}\left(\mathcal{O}_{p}, \mathcal{O}_{q}\right)=1$ when $p=\tau(q)$. In some sense, these two types of extensions generate the entire category $\mathcal{C}_{f, p}$, in a way which will be made precise by the next theorem. 
Let $C_{p}$ be the ring of $\mathbb{Z} \times \mathbb{Z}$ lower triangular matrices with entries in $k[[x]]$. Write $e_{i j}$ for the matrix units in $C_{p}$. Thus $C_{p}$ is a locally noetherian ring, with idempotents $e_{i}=e_{i i}$ and $C_{p} \cong \prod_{i} e_{i} C_{p}$, as right $C_{p}$-modules. Each $e_{i} C_{p}$ has a unique simple factor module $S_{i}$ and we write $S_{i}=C_{p} / \mathfrak{n}_{i}$ for the appropriate ideal $\mathfrak{n}_{i}$. Let $N$ be the matrix with a 1 in the $(i, i-1)$-position for all $i \in \mathbb{Z}$, and 0 's elsewhere. Then $N$ is normal in $C_{p}$ and the ideal $N C_{p}$ consists of all matrices in $C_{p}$ which are 0 along the main diagonal. Let mod- $C_{p}$ be the category of finitely generated right $C_{p}$-modules, and fd- $C_{p}$ the category of $C_{p}$-modules of finite $\mathbb{k}$-dimension.

The next result from [VV, VB2, which relates $\mathcal{C}_{f, p}$ to $\mathrm{fd}-C_{p}$, forms the starting point to this section. It provides a very convenient way of analysing the graded $T$-modules of GK-dimension 1.

Theorem 3.1. Let $X=$ qgr-T for $T$ satisfying Hypothesis 2.2, and keep the above notation. Fix $p \in E$.

(1) There is an equivalence of categories $(\widehat{-})_{p}: \mathcal{C}_{f, p} \rightarrow \mathrm{fd}-C_{p}$. We have $\left(\widehat{\mathcal{O}_{\tau^{i}(p)}}\right)_{p}=S_{i}$ for each $i \in \mathbb{Z}$.

(2) More generally, there is an exact functor $(\widehat{-})_{p}: X=\operatorname{qgr}-T \rightarrow \bmod -C_{p}$, which restricts on $\mathcal{C}_{f, p}$ to the equivalence of Part (1). One has $\left(\widehat{\mathcal{O}_{X}}\right)_{p}=C_{p}$, and for any right ideal $L$ of $T, \widehat{\pi(L g)}_{p}=\widehat{\pi(L)}_{p} N$.

(3) Given a noetherian object $\mathcal{F} \in X,(\widehat{-})_{p}$ defines a one-to-one correspondence between subobjects $\mathcal{G}$ of $\mathcal{F}$ such that $\mathcal{F} / \mathcal{G} \in \mathcal{C}_{f, p}$ and $C_{p}$-submodules $H$ of $\widehat{\mathcal{F}}_{p}$ such that $\widehat{\mathcal{F}}_{p} / H \in \mathrm{fd}-C_{p}$. Under this correspondence, $\mathcal{F} / \mathcal{G} \in Y$ if and only if $\widehat{\mathcal{F}}_{p} N \subseteq \widehat{\mathcal{G}}_{p} \subseteq \widehat{\mathcal{F}}_{p}$. Similarly, under this correspondence $\mathcal{F} / \mathcal{G}$ is a direct sum of copies of $\mathcal{O}_{\tau^{i}(p)}$ if and only if $\widehat{\mathcal{F}}_{p} \mathfrak{n}_{i} \subseteq \widehat{\mathcal{G}}_{p} \subseteq \widehat{\mathcal{F}}_{p}$.

Proof. This is a combination and restatement of several results in [VB2, which are themselves a generalisation of the original result from $[\mathrm{VV}]$. These results are stated in the language of topological rings and modules, since the ring $C_{p}$ is a topological ring with the co-finite-length topology. We will not use this language, other than to explain how our result follows from the results in VV, VB2].

(1) This follows from [VV, Theorem 1.1] or from the more general result [VB2, Theorem 5.1.4].

(2) The functor $(\widehat{-})_{p}$ from Part $(1)$ is extended to an exact functor $X=$ qgr- $T \rightarrow \bmod -C_{p}$ in VB2, Section 5.3]. There the image is taken to lie in the category of pseudo-compact $C_{p}$-modules as defined in VB2, Section 4], but it is also noted that the objects in the image of the functor are finitely generated. The fact that $\left(\widehat{\mathcal{O}_{X}}\right)_{p}=C_{p}$ is $\overline{\mathrm{VB} 2}$, Lemma 5.3.3], and the last statement follows from [VB2, Theorem 5.3.1].

(3) By [VB2, Corollary 5.3.5], $(\widehat{-})_{p}$ induces a bijection between subobjects $\mathcal{G}$ of $\mathcal{F}$ such that $\mathcal{F} / \mathcal{G} \in \mathcal{C}_{f, p}$ and open subobjects of $(\widehat{\mathcal{F}})_{p}$. However, such open subobjects are the same as co-finite-dimensional $C_{p^{-}}$ submodules (use [VB2, Lemma 4.2] and the fact that finite length $C_{p}$-modules are finite-dimensional).

Now by the equivalence of Part $(1),(\mathcal{F} / \mathcal{G})$ is a direct sum of copies of $\mathcal{O}_{\tau^{i}(p)}$ if and only if $\widehat{\mathcal{F}}_{p} / \widehat{\mathcal{G}}_{p}$ is a direct sum of copies of $S_{i}$. The latter condition is equivalent to $\widehat{\mathcal{F}}_{p} \mathfrak{n}_{i} \subseteq \widehat{\mathcal{G}}_{p} \subseteq \widehat{\mathcal{F}}_{p}$, as desired. The other statement is proved similarly: use the fact that an object is in $\mathcal{C}_{f, p} \cap Y$ if and only if its image under $(\widehat{-})_{p}$ is a $C_{p} /(N)$-module $[\mathrm{VB2}$, Theorem $5.1 .4(4,5)]$.

Using Theorem 3.1 we can associate some important right ideals of $T$ to sequences of divisors on $E$. 
Definition 3.2. A finite sequence of effective divisors $\left(\mathbf{d}^{0}, \ldots \mathbf{d}^{k-1}\right)$ on $E$ is called an allowable divisor layering if it satisfies the condition

$$
\tau^{-1}\left(\mathbf{d}^{i-1}\right) \geq \mathbf{d}^{i} \quad \text { for all } 1 \leq i \leq k-1
$$

We adopt the convention that $\mathbf{d}^{m}=0$ for all $m \geq k$, and we use the notation $\mathbf{d}^{\bullet}=\left(\mathbf{d}^{0}, \ldots, \mathbf{d}^{k-1}\right)$ to represent the divisor layering as a whole. If $q \in E$, let $\mathbb{O}(q)=\left\{\tau^{i}(q) \mid i \in \mathbb{Z}\right\}$ be the $\tau$-orbit of $q$.

Definition 3.4. Assume Hypothesis 2.2, and let $\mathbf{d}^{\bullet}$ be an allowable divisor layering. To $\mathbf{d}^{\bullet}$ we will associate a subobject $\mathcal{J}\left(\mathbf{d}^{\bullet}\right)$ of $\mathcal{O}_{X}$, where $X=$ qgr- $T$, and a saturated right ideal $J\left(\mathbf{d}^{\bullet}\right)$ of $T$.

Suppose first that each $\mathbf{d}^{i}$ is supported on $\mathbb{O}(p)$. Then $\mathbf{d}^{i}=\sum_{j} a_{j+i, j} \tau^{j}(p)$ for some unique integers $a_{k, \ell} \geq 0$ and we define $\mathfrak{J}=\mathfrak{J}\left(\mathbf{d}^{i}\right) \subseteq C_{p}$ by putting $(x)^{a_{k, \ell}}$ in the $(k, \ell)$-spot of the lower triangular matrix. To see that $\mathfrak{J}$ is a right ideal, note that, by condition (3.3), $a_{k, \ell} \leq a_{k, \ell+1}$ for all $k, \ell$. Under the correspondence given in Theorem $3.1(3), \mathfrak{J}=\widehat{\mathcal{J}}_{p}$ for a subobject $\mathcal{J}=\mathcal{J}\left(\mathbf{d}^{\bullet}\right)$ of $\mathcal{O}_{X}$ such that $\mathcal{O}_{X} / \mathcal{J}$ has finite length with composition factors among the $\mathcal{O}_{\tau^{i}(p)}$. Thus $J\left(\mathbf{d}^{\bullet}\right)=\omega\left(\mathcal{J}\left(\mathbf{d}^{\bullet}\right)\right)$ is a saturated right ideal of $T$.

Given a general allowable divisor layering, choose representative points $p_{1}, \ldots p_{s}$ on distinct $\tau$-orbits, such that every point involved in $\mathbf{d}^{\bullet}$ lies in $\mathbb{O}\left(p_{k}\right)$, for some $k$. For each $p_{k}$ we define $\mathbf{d}_{k}^{i}=\left.\mathbf{d}^{i}\right|_{\mathbb{O}\left(p_{k}\right)}$. Then $\mathbf{d}_{k}^{\bullet}$ is an allowable divisor layering and we put $\mathcal{J}\left(\mathbf{d}^{\bullet}\right)=\bigcap_{k} \mathcal{J}\left(\mathbf{d}_{k}^{\bullet}\right)$ and $J\left(\mathbf{d}^{\bullet}\right)=\bigcap_{k} J\left(\mathbf{d}_{k}^{\bullet}\right)=\omega\left(\bigcap_{k} \mathcal{J}\left(\mathbf{d}_{k}^{\bullet}\right)\right)$.

The following result explains the idea behind the name divisor layering: each divisor $\mathbf{d}^{j}$ tracks which points (with multiplicities) occur in the $j^{\text {th }}$ layer $M g^{j} / M g^{j+1}$ of the module $M=T / J\left(\mathbf{d}^{\bullet}\right)$.

Lemma 3.5. Let $\mathbf{d}^{\bullet}$ be an allowable divisor layering and let $J=J\left(\mathbf{d}^{\bullet}\right)$ and $M=T / J$.

(1) If $M^{j}=M g^{j} / M g^{j+1}$, then as objects in qgr- $B$ we have

$$
\pi\left(M^{j}\right) \cong \pi\left(\bigoplus_{n \geq 0} H^{0}\left(E,\left(\mathcal{O}_{E} / \mathcal{O}_{E}\left(-\mathbf{d}^{j}\right)\right) \otimes \mathcal{M}_{n}\right)\right) .
$$

In particular, the divisor $\mathbf{d}^{j}$ determines the (tails of) point modules that occur in a filtration of $M^{j}$.

(2) $(\bar{J})^{\text {sat }}=\bigoplus_{n \geq 0} H^{0}\left(E, \mathcal{M}_{n}\left(-\mathbf{d}^{0}\right)\right)$.

(3) If $\mathbf{d}^{\bullet}=(\mathbf{d})$ has length 1 , then $J(\mathbf{d})=\bigoplus_{n \geq 0}\left\{x \in T_{n} \mid \bar{x} \in H^{0}\left(E, \mathcal{M}_{n}(-\mathbf{d})\right)\right\}$.

Proof. (1) By definition, $\mathcal{J}\left(\mathbf{d}^{\bullet}\right)=\bigcap_{k} \mathcal{J}\left(\mathbf{d}_{k}^{\bullet}\right)$ where $\mathbf{d}_{k}^{i}=\left.\mathbf{d}^{i}\right|_{\mathbb{O}\left(p_{k}\right)}$ and the orbits $\mathbb{O}\left(p_{k}\right)$ are distinct. Also, the composition factors of $\mathcal{O}_{X} / \mathcal{J}\left(\mathbf{d}_{k}^{\bullet}\right)$ are among the $\mathcal{O}_{\tau^{i}\left(p_{k}\right)}$ by construction. Thus the $\mathcal{J}\left(\mathbf{d}_{k}^{\bullet}\right)$ must be pairwise comaximal inside $\mathcal{O}_{X}$, and so $\pi(M)=\mathcal{O}_{X} / \mathcal{J}\left(\mathbf{d}^{\bullet}\right) \cong \bigoplus_{k} \mathcal{O}_{X} / \mathcal{J}\left(\mathbf{d}_{k}^{\bullet}\right)$. Since $\bigoplus_{k} \mathcal{O}_{E} / \mathcal{O}_{E}\left(-\mathbf{d}_{k}^{j}\right) \cong$ $\mathcal{O}_{E} / \mathcal{O}_{E}\left(-\mathbf{d}^{j}\right)$, we can reduce to the case that the divisor layers $\mathbf{d}^{i}$ are all supported on a single $\tau$-orbit $\mathbb{O}(p)$.

Now since $\pi(M) \in X$ corresponds via Theorem $3.1(1)$ to $(\widehat{\pi(M)})_{p}=C_{p} / \mathfrak{J}\left(\mathbf{d}^{\bullet}\right)$, it suffices to study the filtration of the latter module by $C_{p} /(N)$-modules. From the definition of the ideal $\mathfrak{J}=\mathfrak{J}\left(\mathbf{d}^{\bullet}\right)$, one sees that writing $\mathbf{d}^{i}=\sum b_{j} \tau^{j}(p)$ gives $\left(\mathfrak{J}+N^{i} C_{p}\right) /\left(\mathfrak{J}+N^{i+1} C_{p}\right) \cong C_{p} /\left(\prod_{j} \mathfrak{n}_{j}^{b_{j}}\right) \cong \bigoplus_{j} C_{p} / \mathfrak{n}_{j}^{b_{j}}$. Using Theorem 3.1 again, the object in qgr- $B \sim \operatorname{coh}(E)$ corresponding to this object under the equivalence $(\widehat{-})_{p}$ is $\mathcal{O}_{E} / \mathcal{O}_{E}\left(-\mathbf{d}^{i}\right)$. 
(2) Applying Part (1) with $j=0$, shows that $M / g M=T /(g T+J)=\bar{T} / \bar{J}$ satisfies

$$
\pi(\bar{T} / \bar{J}) \cong \pi\left(\bigoplus_{n \geq 0} H^{0}\left(E, \mathcal{O}_{E} / \mathcal{O}_{E}\left(-\mathbf{d}^{0}\right) \otimes \mathcal{M}_{n}\right)\right) .
$$

This shows that $\bar{J}$ is equal in large degree to $\bigoplus_{n \geq 0} H^{0}\left(E, \mathcal{O}_{E}\left(-\mathbf{d}^{0}\right) \otimes \mathcal{M}_{n}\right)$. But the latter right ideal is easily seen to be saturated.

(3) Since $J$ is saturated and there is only one layer, $T g \subseteq J$. Now the result follows from Part (2).

We always use the partial ordering on divisors on $E$ where $\mathbf{c} \leq \mathbf{d}$ means that $\mathbf{d}-\mathbf{c}$ is effective. This induces a max and min operation on divisors, where explicitly $\max \left(\sum a_{p} p, \sum b_{p} p\right)=\sum \max \left(a_{p}, b_{p}\right) p$ and similarly for the min. These operations are extended to divisor layerings coordinatewise. It is easy to see that the association of right ideals to divisors respects the lattice structures, as follows.

Lemma 3.6. Let $\mathbf{c}^{\bullet}$ and $\mathbf{d}^{\bullet}$ be allowable divisor layerings, and $\operatorname{set} \mathbf{e}^{\bullet}=\max \left(\mathbf{c}^{\bullet}, \mathbf{d}^{\bullet}\right)$ and $\mathbf{b}^{\bullet}=\min \left(\mathbf{c}^{\bullet}, \mathbf{d}^{\bullet}\right)$. Then $\mathcal{J}\left(\mathbf{c}^{\bullet}\right) \cap \mathcal{J}\left(\mathbf{d}^{\bullet}\right)=\mathcal{J}\left(\mathbf{e}^{\bullet}\right)$ and $\mathcal{J}\left(\mathbf{c}^{\bullet}\right)+\mathcal{J}\left(\mathbf{d}^{\bullet}\right)=\mathcal{J}\left(\mathbf{b}^{\bullet}\right)$. Furthermore, $J\left(\mathbf{c}^{\bullet}\right) \cap J\left(\mathbf{d}^{\bullet}\right)=J\left(\mathbf{e}^{\bullet}\right)$ and $\left(J\left(\mathbf{c}^{\bullet}\right)+J\left(\mathbf{d}^{\bullet}\right)\right)^{\text {sat }}=J\left(\mathbf{b}^{\bullet}\right)$.

Proof. First, it is routine to reduce to the case that $\mathbf{c}^{\bullet}, \mathbf{d}^{\bullet}$, and $\mathbf{e}^{\bullet}$ are supported on a single orbit $\mathbb{O}(p)$.

Considering the right ideals $\mathfrak{J}\left(\mathbf{d}^{\bullet}\right)$ and $\mathfrak{J}\left(\mathbf{c}^{\bullet}\right)$ of $C_{p}$ as in Definition 3.4 we have $\mathfrak{J}\left(\mathbf{c}^{\bullet}\right) \cap \mathfrak{J}\left(\mathbf{d}^{\bullet}\right)=\mathfrak{J}\left(\mathbf{e}^{\bullet}\right)$ because the intersection of two right ideals in $C_{p}$ of the form $\left((x)^{a_{i, j}}\right)$ is clearly formed by taking the maximum of the $a_{i, j}$ at each spot. Similarly, $\mathfrak{J}\left(\mathbf{c}^{\bullet}\right)+\mathfrak{J}\left(\mathbf{d}^{\bullet}\right)=\mathfrak{J}\left(\mathbf{b}^{\bullet}\right)$ because the sum of two such right ideals is formed by taking the minimum of the $a_{i, j}$ at each spot.

The functor $(\widehat{-})_{p}$ of Theorem 3.1 is exact, and so the correspondence of Theorem $3.1(3)$ must preserve intersections and sums of subobjects of a fixed object. Thus $\mathcal{J}\left(\mathbf{c}^{\bullet}\right) \cap \mathcal{J}\left(\mathbf{d}^{\bullet}\right)=\mathcal{J}\left(\mathbf{e}^{\bullet}\right)$ and $\mathcal{J}\left(\mathbf{c}^{\bullet}\right)+\mathcal{J}\left(\mathbf{d}^{\bullet}\right)=$ $\mathcal{J}\left(\mathbf{b}^{\bullet}\right)$, by the definition of the $\mathcal{J}$ 's. Applying the section functor $\omega$ gives the last statement.

Right ideals of the form $J\left(\mathbf{d}^{\bullet}\right)$ represent only some of the right ideals $J$ for which $T / J$ is $g$-torsion with $\operatorname{GKdim}(T / J)=1$. To see this, use Theorem 3.1 to pass to some $C_{p}$. Note that $C_{p} / \mathfrak{n}_{0} \mathfrak{n}_{-1} \cong\left(\begin{array}{l}\mathbb{k} 0 \\ \mathbb{k} k\end{array}\right)$, and so there are infinitely many right ideals $\mathfrak{K} \subset C_{p}$ such that $C_{p} / \mathfrak{K}$ is an essential extension of $S_{-1}$ by $S_{0}$. However only one of these can correspond to some $J\left(\mathbf{d}^{\bullet}\right)$; in this case Lemma 3.5 implies that $\mathbf{d}^{\bullet}=\left(p, \tau^{-1}(p)\right)$.

For the rest of this section, we consider certain functors on $X=$ qgr- $T$ and their actions on the $\mathcal{J}\left(\mathbf{d}^{\bullet}\right)$.

Definition 3.7. Fix a point $p$, and consider Theorem 3.1 applied to the $\tau$-orbit of this point. Given $q=\tau^{i}(p)$, we define a functor $F_{q}: X \rightarrow X$ as follows. For an object $\mathcal{G} \in X$, let $F_{q}(\mathcal{G})$ be the smallest subobject $\mathcal{G}^{\prime}$ of $\mathcal{G}$ such that $\mathcal{G} / \mathcal{G}^{\prime}$ is isomorphic to a direct sum of copies of the simple object $\mathcal{O}_{q}$. That is, by Theorem 3.1 $\mathcal{G}^{\prime}$ is the subobject of $\mathcal{G}$ corresponding under the functor $(\widehat{-})_{p}$ to $\widehat{\mathcal{G}}_{p} \mathfrak{n}_{i}$. Given a morphism $f \in \operatorname{Hom}(\mathcal{G}, \mathcal{H})$, we define $F_{q}(f)=\left.f\right|_{F_{q}(\mathcal{G})} \in \operatorname{Hom}\left(F_{q}(\mathcal{G}), F_{q}(\mathcal{H})\right)$. To see that this is well-defined, note that $\mathcal{G} / f^{-1}\left(F_{q}(\mathcal{H})\right)$ embeds in $\mathcal{H} / F_{q}(\mathcal{H})$ and thus is also a direct sum of copies of $\mathcal{O}_{q}$. Thus $f^{-1}\left(F_{q}(\mathcal{H})\right)$ contains the smallest such subobject $F_{q}(\mathcal{G})$ of $\mathcal{G}$, which implies that $f\left(F_{q}(\mathcal{G})\right) \subseteq F_{q}(\mathcal{H})$.

If $G=F_{q_{1}} \circ \cdots \circ F_{q_{n}}$ is a composition of such functors, write $\tau^{j}(G)$ for the functor $F_{\tau^{j}\left(q_{1}\right)} \circ \cdots \circ F_{\tau^{j}\left(q_{n}\right)}$. 
Notation 3.8. Given an effective divisor $\mathbf{d}$ on $E$, we define a functor $G_{\mathbf{d}}: X \rightarrow X$ as follows. Break $\mathbf{d}$ up by orbit so that $\mathbf{d}=\sum_{j=1}^{n} \mathbf{d}_{j}$, where $\mathbf{d}_{j}$ is supported entirely on $\mathbb{O}\left(p_{j}\right)$ for some $p_{j} \in E$. If needed, reindex $\mathbb{O}\left(p_{j}\right)$ so that $\mathbf{d}_{j}=\sum_{i=0}^{m} b_{i} \tau^{i}\left(p_{j}\right)$ and define $G_{j}=F_{\tau^{m}\left(p_{j}\right)}^{b_{m}} \circ \cdots \circ F_{\tau\left(p_{j}\right)}^{b_{1}} \circ F_{p_{j}}^{b_{0}}$. Finally, set $G_{\mathbf{d}}=G_{1} \circ G_{2} \circ \cdots \circ G_{n}$.

Lemma 3.9. Let $\mathbf{z}^{\bullet}=\left(\mathbf{z}^{0}, \ldots, \mathbf{z}^{k-1}\right)$ be an allowable divisor layering, and let $\mathcal{J}=\mathcal{J}\left(\mathbf{z}^{\bullet}\right)$. Let $\mathbf{d}$ be an effective divisor on $E$. Then $G_{\mathbf{d}}(\mathcal{J})$ is defined by the allowable divisor layering $\mathbf{x}^{\bullet}=\left(\mathbf{x}^{0}, \ldots, \mathbf{x}^{k}\right)$, where

$$
\mathbf{x}^{i}= \begin{cases}\mathbf{z}^{0}+\mathbf{d} & \text { if } i=0 \\ \min \left(\mathbf{z}^{i}+\mathbf{d}, \tau^{-1}\left(\mathbf{z}^{i-1}\right)\right) & \text { if } 1 \leq i \leq k\end{cases}
$$

Proof. We prove this by induction. If $\mathbf{d}=p$ is a single point, this is basically a restatement of VB2, Proposition 5.2.2]. To see this, note that, by construction, $\mathbf{x}^{\bullet}$ is allowable; note that since $\mathbf{z}^{i} \leq \tau^{-1} \mathbf{z}^{i-1}$, therefore $\mathbf{z}^{i}+p \leq \tau^{-1}\left(\mathbf{z}^{i-1}+p\right)$ if and only if $\mathbf{z}^{i}+p \leq \tau^{-1}\left(\mathbf{z}^{i-1}\right)$. We must prove that $\mathbf{x}^{\bullet}$ defines $F_{p} \mathcal{J}$.

It is clear from the definition of $F_{p}$ that to compute $F_{p}(\mathcal{J})$ we only need to consider the orbit of $p$ : that is, without loss of generality we may assume that $\mathbf{z}^{0}$ is supported on the orbit of $p$. Define natural numbers $a_{\ell, m}$ by writing $\mathbf{z}^{i}=\sum_{j} a_{j+i, j} \tau^{j}(p)$. Then $\widehat{\mathcal{J}}_{p}=\prod_{\ell \in \mathbb{Z}} P_{\ell}$, where $P_{\ell} \subseteq e_{\ell} C_{p}$ is the infinite row vector defined by putting $(x)^{a_{\ell, m}}$ in the $(\ell, m)$ spot. As remarked in the definition of $\mathcal{J}\left(\mathbf{d}^{\bullet}\right)$, each $P_{\ell}$ is a right ideal of $C_{p}$.

The object $F_{p} \mathcal{J}$ corresponds under $(\widehat{-})_{p}$ to $\widehat{\mathcal{J}}_{p} \cdot \mathfrak{n}_{0}=\prod_{\ell} P_{\ell} \cdot \mathfrak{n}_{0}$. But $P_{\ell} \cdot \mathfrak{n}_{0}$ is easy to describe: namely, by VB2, Proposition 5.2.2] or a direct computation, $P_{\ell} \cdot \mathfrak{n}_{0}$ is defined by $(x)^{b_{\ell, m}}$ where

$$
b_{\ell, m}= \begin{cases}a_{\ell, m} & \text { if } m \neq 0 \\ a_{\ell, 0}+1 & \text { if } a_{\ell, 0}<a_{\ell, 1} \text { or if } \ell=0 \\ a_{\ell, 0} & \text { if } \ell \geq 1 \text { and } a_{\ell, 0}=a_{\ell, 1} .\end{cases}
$$

Now let $a_{\ell, m}^{\prime}$ be defined by $\mathbf{x}^{i}=\sum_{j} a_{i+j, j}^{\prime} \tau^{j}(p)$. The $a_{\ell, m}^{\prime}$ satisfy $a_{0,0}^{\prime}=a_{0,0}+1, a_{\ell, m}^{\prime}=a_{\ell, m}$ if $m \neq 0$, and if $\ell>0$ then

$$
a_{\ell, 0}^{\prime}= \begin{cases}a_{\ell, 0}+1 & \text { if } \tau^{-1}\left(\mathbf{z}^{\ell-1}\right) \geq \mathbf{z}^{\ell}+p, \text { that is, if } a_{\ell, 1}>a_{\ell, 0} \\ a_{\ell, 0} & \text { otherwise. }\end{cases}
$$

We see that $a_{\ell, m}^{\prime}=b_{\ell, m}$. It follows from Theorem 3.1 that $F_{p} \mathcal{J}=\mathcal{J}\left(\mathbf{x}^{\bullet}\right)$.

Now assume that $\operatorname{deg} \mathbf{d} \geq 2$. Choose $q \in E$ and an effective divisor $\mathbf{d}^{\prime}$ on $E$ so that $\mathbf{d}=q+\mathbf{d}^{\prime}$ and $\tau^{-1}\left(\mathbf{d}^{\prime}\right)$ does not contain $q$. Note that $G_{\mathbf{d}}=F_{q} \circ G_{\mathbf{d}^{\prime}}$. By induction, $G_{\mathbf{d}^{\prime}} \mathcal{J}$ is defined by the allowable divisor layering

$$
\mathbf{y}^{i}= \begin{cases}\mathbf{z}^{0}+\mathbf{d}^{\prime} & \text { if } i=0 \\ \min \left(\mathbf{z}^{i}+\mathbf{d}^{\prime}, \tau^{-1}\left(\mathbf{z}^{i-1}\right)\right) & 1 \leq i \leq k .\end{cases}
$$

Applying the one-point case, we find that $G_{\mathbf{d}}(\mathcal{J})$ is defined by

$$
\mathbf{x}^{i}= \begin{cases}\mathbf{z}^{0}+\mathbf{d}^{\prime}+q=\mathbf{z}_{0}+\mathbf{d} & \text { if } i=0 \\ \min \left(\mathbf{z}^{1}+\mathbf{d}, \tau^{-1}\left(\mathbf{z}^{0}\right)+q, \tau^{-1}\left(\mathbf{z}^{0}+\mathbf{d}^{\prime}\right)\right) & \text { if } i=1 \\ \min \left(\mathbf{z}^{i}+\mathbf{d}, \tau^{-1}\left(\mathbf{z}^{i-1}\right)+q, \tau^{-1}\left(\mathbf{z}^{i-1}+\mathbf{d}^{\prime}\right), \tau^{-2}\left(\mathbf{z}^{i-2}\right)\right) & \text { if } 2 \leq i \leq k+1\end{cases}
$$


and that this is allowable. Since $\tau^{-1}\left(\mathbf{d}^{\prime}\right)$ does not meet $q$ and $\mathbf{z}^{i-1} \leq \tau^{-1}\left(\mathbf{z}^{i-2}\right)$, this simplifies to

$$
\mathbf{x}^{i}= \begin{cases}\mathbf{z}^{0}+\mathbf{d} & \text { if } i=0 \\ \min \left(\mathbf{z}^{i}+\mathbf{d}, \tau^{-1}\left(\mathbf{z}^{i-1}\right)\right) & \text { if } 1 \leq i \leq k+1 .\end{cases}
$$

Finally, since $\mathbf{z}^{k}=0$, therefore $\mathbf{x}^{k+1}=0$. The lemma is proved.

We also extend the previous result to calculate the result of applying certain compositions of $G_{\mathbf{d}}$ 's.

Lemma 3.10. Let $\mathbf{d}$ be an effective divisor, and let $\mathbf{d}_{n}=\mathbf{d}+\tau^{-1} \mathbf{d}+\cdots+\tau^{-(n-1)} \mathbf{d}$ if $n \geq 1$. If $n \leq 0$, define $\mathbf{d}_{n}=0$. Let $\mathcal{J}=\mathcal{J}\left(\mathbf{z}^{\bullet}\right)$, where $\mathbf{z}^{\bullet}$ is an allowable divisor sequence.

Define $G_{\mathbf{d}}$ as in Notation 3.8. Then for any $n \in \mathbb{N}$, the object $\left(\tau^{-(n-1)} G_{\mathbf{d}} \circ \cdots \circ \tau^{-1} G_{\mathbf{d}} \circ G_{\mathbf{d}}\right)(\mathcal{J})$ is equal to $\mathcal{J}\left(\mathbf{w}^{\bullet}\right)$, where

$$
\mathbf{w}^{i}=\min _{j=0}^{i}\left(\tau^{-j}\left(\mathbf{z}^{i-j}+\mathbf{d}_{n-j}\right)\right) .
$$

Proof. The case $n=1$ is Lemma 3.9, since $\tau^{-1}\left(\mathbf{z}^{i-1}\right) \leq \tau^{-2}\left(\mathbf{z}^{i-2}\right) \leq \ldots$ Assume by induction that $\left(\tau^{-(n-2)} G_{\mathbf{d}} \circ \cdots \circ G_{\mathbf{d}}\right)(\mathcal{J})$ is defined by $\mathbf{x}^{\bullet}$, where

$$
\mathbf{x}^{i}=\min _{j=0}^{i}\left(\tau^{-j}\left(\mathbf{z}^{i-j}+\mathbf{d}_{n-1-j}\right)\right) .
$$

Applying Lemma 3.9, we obtain that $\mathbf{w}^{0}=\mathbf{z}^{0}+\mathbf{d}_{n-1}+\tau^{-(n-1)} \mathbf{d}=\mathbf{z}^{0}+\mathbf{d}_{n}$, and for $i \geq 1$ we have

$$
\begin{aligned}
\mathbf{w}^{i}= & \min \left(\mathbf{x}^{i}+\tau^{-(n-1)} \mathbf{d}, \tau^{-1}\left(\mathbf{x}^{i-1}\right)\right) \\
& =\min \left(\min _{j=0}^{i}\left(\tau^{-j}\left(\mathbf{z}^{i-j}+\mathbf{d}_{n-1-j}\right)+\tau^{-(n-1)}(\mathbf{d})\right), \min _{j=0}^{i-1}\left(\tau^{-j-1}\left(\mathbf{z}^{i-1-j}+\mathbf{d}_{n-1-j}\right)\right)\right) .
\end{aligned}
$$

Since $\tau^{-j}\left(\mathbf{d}_{n-1-j}\right)+\tau^{-(n-1)}(\mathbf{d})=\tau^{-j}\left(\mathbf{d}_{n-j}\right)$, this expression reduces to the desired expression for $\mathbf{w}^{i}$.

\section{Certain Right ideals of $T$ And their Hilbert series}

We continue to assume throughout this section that $T$ is a ring satisfying Hypothesis 2.2 ,

Starting in this section, we work more directly in the graded $\operatorname{ring} T$. We begin by defining an important special case of the right ideals $J\left(\mathbf{d}^{\bullet}\right)$, which will be crucial in describing the blowups $T(\mathbf{d})$ later. Recall the notation $\mathbf{d}_{n}=\mathbf{d}+\tau^{-1}(\mathbf{d})+\cdots+\tau^{-n+1}(\mathbf{d})$ from Section 2 .

Definition 4.1. Fix an effective divisor $\mathbf{d}$ on $E$ and for each $k \geq 1$, let $M(k, \mathbf{d})$ be the ideal $J\left(\mathbf{d}^{\bullet}\right)$ for the (necessarily allowable) divisor layering $\mathbf{d}^{\bullet}=\left(\mathbf{d}^{0}, \mathbf{d}^{1}, \ldots, \mathbf{d}^{k-1}\right)$, where

$$
\mathbf{d}^{0}=\mathbf{d}_{k}, \quad \mathbf{d}^{1}=\tau^{-1}\left(\mathbf{d}_{k-1}\right), \ldots, \mathbf{d}^{k-1}=\tau^{-k+1}(\mathbf{d}) .
$$

By convention, if $k \leq 0$, then $M(k, \mathbf{d})=T$, regardless of $\mathbf{d}$.

Note that $M(k, \mathbf{d})$ and $J\left(\mathbf{d}^{\bullet}\right)$ depend on the ambient ring $T$, and if this ring is not clear from the context, we will write $M(k, \mathbf{d})=M_{T}(k, \mathbf{d})$ and $J\left(\mathbf{d}^{\bullet}\right)=J_{T}\left(\mathbf{d}^{\bullet}\right)$.

The $M(k, \mathbf{d})$ can be easily described in terms of the functors developed in the previous section, as follows. 
Lemma 4.2. Let $\mathbf{d}$ be an effective divisor on $E$, and let $G_{\mathbf{d}}$ be the functor defined in Notation 3.8. If $M(k, \mathbf{d})$ is defined as above, then its image $\pi(M(k, \mathbf{d})) \in X$ is equal to

$$
\left[\tau^{-k+1}\left(G_{\mathbf{d}}\right) \circ \tau^{-k+2}\left(G_{\mathbf{d}}\right) \circ \cdots \circ \tau^{-1}\left(G_{\mathbf{d}}\right) \circ G_{\mathbf{d}}\right]\left(\mathcal{O}_{X}\right) .
$$

Proof. This follows directly from Lemma 3.10

We want to study the properties of the right ideals $M(k, \mathbf{d})$, and our first goal is to compute their Hilbert series precisely. We start with a routine lemma.

Lemma 4.3. If $J$ is a saturated right ideal of $T$ such that $M=T / J$ is $g$-torsion with $\operatorname{GKdim} M=1$, then $M$ has a filtration whose factors are shifted point modules. Also $\operatorname{dim}_{\mathbb{k}} M_{n} \leq \operatorname{dim}_{\mathbb{k}} M_{n+1}$ for all $n \in \mathbb{Z}$.

Proof. As $M$ has no finite-dimensional submodules, it has a finite chain of submodules, each factor of which is 1-critical (see [MR, (6.2.19) and (6.8.25)]) and by induction it suffices to prove the result when $M$ is 1critical. As noted in [RSS, Lemma 3.8], $M$ is then killed by $g$ and so $M$ is a module over $T / g T \cong B(E, \mathcal{M}, \tau)$. Since $T / g T$ is generated in degree 1 (see Hypothesis 2.2), the result follows from Lemma 2.8(3).

Lemma 4.4. Let $\mathbf{d}^{\bullet}=\left(\mathbf{d}^{0}, \ldots, \mathbf{d}^{k-1}\right)$ be an allowable divisor layering. Set $J=J\left(\mathbf{d}^{\bullet}\right)$ and $s=\sum_{i} \operatorname{deg} \mathbf{d}^{i}$.

(1) $\operatorname{dim}_{\mathbb{k}}(T / J)_{n} \leq s$ for all $n \geq 0$, with equality for $n \gg 0$.

(2) Let $L=J\left(\mathbf{e}^{\bullet}\right)$ where $\mathbf{e}^{\bullet}=\left(\tau\left(\mathbf{d}^{1}\right), \tau\left(\mathbf{d}^{2}\right), \ldots, \tau\left(\mathbf{d}^{k-1}\right)\right)$. Then $J \cap T g=L g$ and

$$
h_{T / J}(t)=h_{\bar{T} / \bar{J}}(t)+t h_{T / L}(t) .
$$

(3) Suppose that $\operatorname{deg} \mathbf{d}^{i}<\mu(\ell-i)$ for all $0 \leq i \leq k-1$, and some $\ell \geq k$. Then $\operatorname{dim}_{\mathbb{k}}(T / J)_{n}=s$ for all $n \geq \ell$ and $\bar{J}_{n}=\bigoplus_{n \geq 0} H^{0}\left(E, \mathcal{M}_{n}\left(-\mathbf{d}^{0}\right)\right)$ for $n \geq \ell$.

Proof. (1) By Lemma 4.3, $M$ has a filtration whose factors which are shifted point modules. By Lemma 3.5 the sum of all of the $\mathbf{d}^{i}$ counts with multiplicity the number of shifted point modules which occur in this filtration. Thus $\operatorname{dim}_{\mathbb{k}}(T / J)_{n}=s$ for $n \gg 0$ and so, by Lemma 4.3, $\operatorname{dim}_{\mathbb{k}}(T / J)_{n} \leq s$ for all $n \geq 0$.

(2) Define $L$ by $L g=J \cap T g$. Thus $L$ is a saturated right ideal of $T$ and we first need to prove that $L=J\left(\mathbf{e}^{\bullet}\right)$ for the given $\mathbf{e}^{\bullet}$. To see this, reduce to the case that the divisors $\mathbf{d}^{i}$ are all supported on a single orbit $\mathbb{O}(p)$. In $X$, we have $\pi(L g)=\pi(J) \cap \pi(T g)$. By the proof of Lemma 3.6 the functor $(\hat{-})_{p}$ of Theorem 3.1 preserves intersections. Applying this functor to our equation and using Theorem 3.1 (2) gives $\mathfrak{L} N=\mathfrak{J}\left(\mathbf{d}^{\bullet}\right) \cap N C_{p}$, where $\mathfrak{L}=(\widehat{\pi(L)})_{p}$. By definition, $\mathfrak{J}\left(\mathbf{d}^{\bullet}\right) \cap N C_{p}$ has 0 in each $(k, k)$-spot, and $(x)^{a_{k, \ell}}$ in the $(k, \ell)$-spots with $k>\ell$, where $\mathbf{d}^{i}=\sum_{j} a_{j+i, j} \tau^{j}(p)$. On the other hand,

$$
\mathbf{e}^{i}=\tau\left(\mathbf{d}^{i+1}\right)=\sum_{j} a_{j+i+1, j} \tau^{j+1}(p)=\sum_{j} a_{j+i, j-1} \tau^{j}(p) .
$$

Then $\mathfrak{J}\left(\mathbf{e}^{\bullet}\right)$ has $(x)^{a_{k, \ell-1}}$ in the $(k, \ell)$-spot, and so a calculation shows that $\mathfrak{J}\left(\mathbf{e}^{\bullet}\right) N$ has $(x)^{a_{k, \ell}}$ in the $(k, \ell)$ spot for $k>\ell$ and 0 in each $(k, k)$-spot. Hence $\mathfrak{J}\left(\mathbf{d}^{\bullet}\right) \cap N C_{p}=\mathfrak{J}\left(\mathbf{e}^{\bullet}\right) N$. Thus $\mathfrak{L}=\mathfrak{J}\left(\mathbf{e}^{\bullet}\right)$. So $\pi(L)=\pi\left(J\left(\mathbf{e}^{\bullet}\right)\right)$, and taking saturations gives $L=J\left(\mathbf{e}^{\bullet}\right)$, as claimed. 
In order to compute (4.5), use the exact sequence $0 \rightarrow T g /(J \cap T g) \rightarrow T / J \rightarrow T /(J+T g) \rightarrow 0$, noting that $T g /(J \cap T g)=T g / L g \cong(T / L)[-1]$ and $T /(J+T g) \cong \bar{T} / \bar{J}$.

(3) By Part (1), to show $\operatorname{dim}_{\mathbb{k}}(T / J)_{n}=s$ for all $n \geq \ell$, it is enough to show that $\operatorname{dim}_{\mathbb{k}}(T / J)_{n} \geq s$ if $n \geq \ell$. By Lemma 3.5 (2), $\bar{J}^{\text {sat }}=\bigoplus_{n \geq 0} H^{0}\left(E, \mathcal{M}_{n}\left(-\mathbf{d}^{0}\right)\right)$. By assumption $\operatorname{deg} \mathbf{d}^{0}<(\operatorname{deg} \mathcal{M}) \ell \leq \operatorname{deg} \mathcal{M}_{n}$ for $n \geq \ell$, and so the divisor $\mathbf{d}^{0}$ presents independent vanishing conditions to sections of the sheaf $\mathcal{M}_{n}$ for $n \geq \ell$; in other words, $\operatorname{dim}_{\mathbb{k}} H^{0}\left(E, \mathcal{M}_{n}\left(-\mathbf{d}^{0}\right)\right)=\operatorname{dim}_{\mathbb{k}} H^{0}\left(E, \mathcal{M}_{n}\right)-\operatorname{deg} \mathbf{d}^{0}$. Thus $\operatorname{dim}_{\mathbb{k}}(\bar{T} / \bar{J})_{n} \geq \operatorname{deg} \mathbf{d}^{0}$ for $n \geq \ell$.

Let $L g=J \cap T g$ as in Part (2). Then $L=J\left(\mathbf{e}^{\bullet}\right)$ in the notation of that part and hence $L$ has fewer divisor layers; the divisor layering $\mathbf{e}^{\bullet}$ also satisfies $\operatorname{deg} \mathbf{e}^{i}=\operatorname{deg} \mathbf{d}^{i+1}<(\operatorname{deg} \mathcal{M})(\ell-i-1)$ for $0 \leq i \leq k-2$. By induction we have $\operatorname{dim}_{\mathrm{k}}(T / L)_{n}=\sum_{i=0}^{k-2} \operatorname{deg} \mathbf{e}^{i}=\sum_{i=1}^{k-1} \operatorname{deg} \mathbf{d}^{i}$ for $n \geq \ell-1$. Now by Part (2), we see that $\operatorname{dim}_{\mathbb{k}}(T / J)_{n} \geq s$ for $n \geq \ell$, and hence $\operatorname{dim}_{\mathbb{k}}(T / J)_{n}=s$ for $n \geq \ell$. Examining the proof above, this also shows that the inequality $\operatorname{dim}_{\mathbb{k}}(\bar{T} / \bar{J})_{n} \geq \operatorname{deg} \mathbf{d}^{0}$ for $n \geq \ell$ must have been an equality for each $n \geq \ell$, or equivalently that $\bar{J}$ must have been saturated in degrees $\geq \ell$, proving the second part.

When applied to the special case of the right ideals $M(k, \mathbf{d})$ of $T$ as defined in Definition 4.1, Lemma 4.4 gives the following result.

Proposition 4.6. Let $\mathbf{d}$ be an effective divisor on $E$ with $\operatorname{deg} \mathbf{d}<\operatorname{deg} \mathcal{M}$. Then for all $0 \leq k \leq n$, we have:

(1) $\operatorname{dim} M(k, \mathbf{d})_{n}=\operatorname{dim} T_{n}-(\operatorname{deg} \mathbf{d})\left(\begin{array}{c}k+1 \\ 2\end{array}\right)$.

(2) $(M(k, \mathbf{d}) \cap g T)_{n}=g M(k-1, \mathbf{d})_{n-1}$.

(3) $\overline{M(k, \mathbf{d})}_{n}=H^{0}\left(E, \mathcal{M}_{n}\left(-\mathbf{d}_{k}\right)\right)$.

Remark 4.7. Using the convention that $M(k, \mathbf{d})=T$ if $k \leq 0$, Proposition 4.6(2) is easily seen to hold for all $k \leq n \in \mathbb{Z}$.

Using the functors developed in the Section 3, we next show that a product of two graded pieces of right ideals of the form $J\left(\mathbf{d}^{\bullet}\right)$ is sometimes again a graded piece of some $J\left(\mathbf{c}^{\bullet}\right)$.

Recall that a graded vector subspace $V \subseteq T$ is $g$-divisible if $V \cap T g=V g$. Given graded modules $M, N \subseteq P$ over a cg algebra $A$, we write $M \doteq N$ if $M$ and $N$ agree up to a finite dimensional vector space. Finally, recall that $\omega:$ qgr- $A \rightarrow \operatorname{Gr}-A$ denotes the section functor.

Lemma 4.8. Let $X=\operatorname{qgr}-T$.

(1) Let $\Sigma=[1]$ be the shift functor on $X$. Then $\Sigma \circ F_{p}=F_{\tau(p)} \circ \Sigma$.

(2) Let $H$ be any finite composition of functors of the form $F_{q}$ for $q \in E$. Then

$$
\omega(\mathcal{G})_{m} \cdot \omega\left(H\left(\mathcal{O}_{X}\right)\right)_{n} \subseteq \omega\left(\left[\tau^{-m}(H)\right](\mathcal{G})\right)_{n+m}
$$

for any noetherian object $\mathcal{G} \in X$ and any $m, n \geq 1$.

Proof. (1) This is equivalent to the following statement: If $P(p)$ is the point module of $T$ corresponding to the point $p \in E$, as defined before Lemma 2.8, then $P(p)[1] \stackrel{\ominus}{\doteq}(\tau(p))$. This follows from a direct calculation. 
(2) Set $\widetilde{T}=\omega(\pi(T))$. In Lemma 2.5 we noted that $\widetilde{T}=T$, but here we need the explicit multiplication rule for $\widetilde{T}$. From [AZ1, Section 4] $\widetilde{T}=\bigoplus_{n \geq 0} \operatorname{Hom}_{X}\left(\mathcal{O}_{X}, \mathcal{O}_{X}[n]\right)$, with its multiplication $\star$ defined by $f \star g=\Sigma^{n}(f) \circ g \in \operatorname{Hom}_{X}\left(\mathcal{O}_{X}, \Sigma^{m+n}\left(\mathcal{O}_{X}\right)\right)$ for $f \in \widetilde{T}_{m}$ and $g \in \widetilde{T}_{n}$.

Let $f \in \omega(\mathcal{G})_{m}=\operatorname{Hom}_{X}\left(\mathcal{O}_{X}, \Sigma^{m}(\mathcal{G})\right)$, and $g \in \omega\left(H\left(\mathcal{O}_{X}\right)\right)_{n}=\operatorname{Hom}_{X}\left(\mathcal{O}_{X}, \Sigma^{n}\left(H\left(\mathcal{O}_{X}\right)\right)\right) \subseteq T_{n}$. Then, using the functoriality of $H$, one sees that $f \star g \in \omega(\mathcal{G})_{n+m}$ is the element

$$
\mathcal{O}_{X} \stackrel{g}{\longrightarrow} \Sigma^{n}\left(H\left(\mathcal{O}_{X}\right)\right) \stackrel{\Sigma^{n}(H(f))}{\longrightarrow}\left[\Sigma^{n} \circ H \circ \Sigma^{m}\right](\mathcal{G}) .
$$

Since $H \circ \Sigma^{m}=\Sigma^{m} \circ \tau^{-m}(H)$ by Part (1),

$$
f \star g \in \operatorname{Hom}_{X}\left(\mathcal{O}_{X},\left[\Sigma^{n+m} \circ \tau^{-m}(H)\right](\mathcal{G})\right)=\omega\left(\tau^{-m}(H)(\mathcal{G})\right)_{n+m},
$$

as required.

In particular, we can apply the result above to the right ideals $M(k, \mathbf{d})$ of $T$.

Lemma 4.9. Let $\mathbf{d}$ be an effective divisor on $E$.

(1) Let $n, m, k, \ell \in \mathbb{N}$. Then

$$
M(k, \mathbf{d})_{m} M\left(\ell, \tau^{m-k}(\mathbf{d})\right)_{n} \subseteq M(k+\ell, \mathbf{d})_{m+n} .
$$

(2) If $\operatorname{deg} \mathcal{M}-\operatorname{deg} \mathbf{d} \geq 2$, then (4.10) is an equality for all $k, \ell \geq 0$ and all $m \geq k, n \geq \ell$. If $\operatorname{deg} \mathcal{M}-\operatorname{deg} \mathbf{d}=1$, then (4.10) is an equality for all $k, \ell \geq 0$ and $m \geq \max \{2, k\}$ and $n \geq \max \{2, \ell\}$.

Proof. (1) Let $G_{\mathbf{d}}$ be the functor defined in Notation 3.8. Note that $\mathcal{G}=\pi(M(k, \mathbf{d}))=G^{\prime}\left(\mathcal{O}_{X}\right)$, where $G^{\prime}=\tau^{-k+1}\left(G_{\mathbf{d}}\right) \circ \tau^{-k+2}\left(G_{\mathbf{d}}\right) \circ \cdots \circ G_{\mathbf{d}}$, by Lemma 4.2, Similarly, defining $H=\tau^{m-k-\ell+1}\left(G_{\mathbf{d}}\right) \circ \cdots \circ \tau^{m-k}\left(G_{\mathbf{d}}\right)$, then $H\left(\mathcal{O}_{X}\right)=\pi\left(M\left(\ell, \tau^{m-k}(\mathbf{d})\right)\right)$.

Now apply Lemma 4.8 (2) to $\mathcal{G}$ and $H$. Since $\tau^{-m}(H) \circ G^{\prime}=\tau^{-k-\ell+1}\left(G_{\mathbf{d}}\right) \circ \cdots \circ G_{\mathbf{d}}$, the result follows.

(2) Consider the inclusion (4.10). If we prove for some values of $k, \ell, m, n$ both that

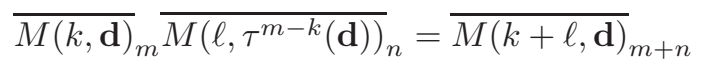

and that

$$
\left(M(k, \mathbf{d})_{m} M\left(\ell, \tau^{m-k}(\mathbf{d})\right)_{n}\right) \cap g T=M(k+\ell, \mathbf{d})_{m+n} \cap g T,
$$

then both sides of (4.10) will have the same vector space dimension and so the inclusion will be an equality.

We start with (4.12). Recall from Remark 4.7 that Proposition 4.6(2) actually holds for all $k \in \mathbb{Z}$ with $k \leq n$. We now allow negative $k$ and $\ell$ in equation (4.12), though we continue to assume that $m, n \geq 0$. This will allow us to prove (4.12) by induction on $m+n$. The base case $m=n=0$ is trivial. If $m \geq 1$, then

$$
\begin{gathered}
M(k, \mathbf{d})_{m} M\left(\ell, \tau^{m-k}(\mathbf{d})\right)_{n} \cap g T \supseteq g M(k-1, \mathbf{d})_{m-1} M\left(\ell, \tau^{m-k}(\mathbf{d})\right)_{n} \\
=g M(k+\ell-1, \mathbf{d})_{m+n-1}=M(k+\ell, \mathbf{d})_{m+n} \cap g T,
\end{gathered}
$$

where we have used Proposition 4.6(2) and the induction hypothesis. The case $n \geq 1$ is similar. 
Thus it remains to consider 4.11). By Proposition 4.6(3),

$$
\begin{gathered}
{\overline{M(k, \mathbf{d})_{m}}}_{m}=H^{0}\left(E, \mathcal{M}_{m}\left(-\mathbf{d}_{k}\right)\right), \quad{\overline{M\left(\ell, \tau^{m-k}(\mathbf{d})\right)_{n}}}=H^{0}\left(E, \mathcal{M}_{n}\left(-\tau^{m-k}\left(\mathbf{d}_{\ell}\right)\right)\right), \\
\text { and }{\overline{M(k+\ell, \mathbf{d})_{n+m}}}_{n}=H^{0}\left(E, \mathcal{M}_{n+m}\left(-\mathbf{d}_{k+\ell}\right)\right) .
\end{gathered}
$$

Set $\mathcal{N}=\mathcal{M}_{m}\left(-\mathbf{d}_{k}\right)$ and $\mathcal{P}=\mathcal{M}_{n}\left(-\tau^{m-k}\left(\mathbf{d}_{\ell}\right)\right)$. By the definition of multiplication in $B(E, \mathcal{M}, \tau)$ the goal is to show that the natural multiplication map

$$
H^{0}(E, \mathcal{N}) \otimes H^{0}\left(E, \mathcal{P}^{\tau^{m}}\right) \rightarrow H^{0}\left(E, \mathcal{N} \otimes \mathcal{P}^{\tau^{m}}\right)
$$

is surjective. Recall from Lemma 2.1 that this is always true provided

$$
2 \leq \operatorname{deg} \mathcal{N}=m \operatorname{deg} \mathcal{M}-k \operatorname{deg} \mathbf{d} \quad \text { and } \quad 2 \leq \operatorname{deg} \mathcal{P}^{\tau^{m}}=n \operatorname{deg} \mathcal{M}-\ell \operatorname{deg} \mathbf{d}
$$

except that if $\operatorname{deg} \mathcal{N}=\operatorname{deg} \mathcal{P}^{\tau^{m}}=2$ one requires the additional condition that $\mathcal{N} \not \mathcal{P}^{\tau^{m}}$. Since (4.13) always holds, it remains to check that this final assertion is also true. This routine calculation is left to the reader: although there are a number of special cases, they all reduce to the fact that $\mathcal{N} ¥ \mathcal{N}^{\tau^{m}}$ for $m \geq 1$.

The next result, which will be used in [RSS, illustrates the usefulness of Lemma 4.9.

Corollary 4.14. Let $\mathbf{d}$ be an effective divisor on $E$ of degree $\leq \mu-2$, and let $k \in \mathbb{N}$. Then

$$
T_{1} M(k, \mathbf{d})_{k}=M\left(k, \tau^{-1}(\mathbf{d})\right)_{k} T_{1}
$$

Proof. By Lemma $4.9(2)$,

$$
M\left(0, \tau^{-1}(\mathbf{d})\right)_{1} M(k, \mathbf{d})_{k}=M\left(k, \tau^{-1}(\mathbf{d})\right)_{k+1}=M\left(k, \tau^{-1}(\mathbf{d})\right)_{k} M\left(0, \tau^{-1}(\mathbf{d})\right)_{1}
$$

\section{The Blowups $T(\mathbf{d})$}

Throughout this section $T$ will satisfy Hypothesis 2.2 Here we define algebras $T(\mathbf{d})$ that correspond to blowing up $T$ at the points on an effective divisor $\mathbf{d}$, and give a uniform proof of their properties.

Definition 5.1. For any effective divisor $\mathbf{d}$ on $E$ with $\operatorname{deg} \mathbf{d}<\mu$, define a subalgebra $T(\mathbf{d})$ of $T$ by putting $T(\mathbf{d})=\bigoplus_{k \geq 0} M(k, \mathbf{d})_{k} \subseteq T$. More generally, for any $\ell \geq 0$ define a graded $T(\mathbf{d})$-submodule of $T$ by

$$
T_{\leq \ell} * T(\mathbf{d}):=\bigoplus_{n \geq 0} M\left(n-\ell, \mathbf{d}^{\tau^{\ell}}\right)_{n}
$$

The facts that $T(\mathbf{d})$ is a subalgebra of $T$, and then that $T_{\leq \ell} * T(\mathbf{d})$ is a right $T(\mathbf{d})$-module, follow immediately from Part (1) of Lemma 4.9.

The notation $T_{\leq \ell} * T(\mathbf{d})$ is meant to recall multiplication, since this module equals $T_{\leq \ell} T(\mathbf{d})$ except when $\operatorname{deg} \mathbf{d}$ is large. We will prove this and other properties in the next few results. 
One should think of $T(\mathbf{d})$ as a ring-theoretic blowup of $T$ along the divisor $\mathbf{d}$. This is justified in part by the properties proven in the next few sections (see Proposition 7.13 and Remark 7.14 among others). The analogy is particularly strong when $\operatorname{deg} \mathbf{d}<\mu-1$, so $T(\mathbf{d})$ is generated in degree one, as the algebra then corresponds to the (commutative) definition of a blowup in terms of Rees rings. It is still appropriate for general $\mathbf{d}$ since one can show that $T(\mathbf{d})$ is the same as the section ring of the blowup defined by Van den Bergh [VB2] (see, in particular, the del Pezzo surfaces considered in [VB2, Section 11]). A further discussion on this analogy can also be found in $[\mathrm{Ro}$.

Proposition 5.2. Let $\mathbf{d}$ be an effective divisor on $E$ with $d=\operatorname{deg} \mathbf{d}<\mu$. Let $n, \ell \in \mathbb{N}$. Then

(1) $\operatorname{dim}_{\mathbb{k}}\left(T_{\leq \ell} * T(\mathbf{d})\right)_{n}=\operatorname{dim}_{\mathbb{k}} T_{n}-d\left(\begin{array}{c}n-\ell+1 \\ 2\end{array}\right)$.

(2) $\left(T_{\leq \ell} * T(\mathbf{d}) \cap g T\right)_{n}=g\left(T_{\leq \ell} * T(\mathbf{d})\right)_{n-1}$; that is, $T_{\leq \ell} * T(\mathbf{d})$ is $g$-divisible.

(3) ${\overline{T_{\leq \ell} * T(\mathbf{d})_{n}}}_{n}=H^{0}\left(E, \mathcal{M}_{n}\left(-\mathbf{d}^{\tau^{\ell}}-\cdots-\mathbf{d}^{\tau^{n-1}}\right)\right)$.

Proof. This is immediate from Proposition 4.6

Theorem 5.3. Let $T$ satisfy Hypothesis 2.2, and let $\mathbf{d}$ be an effective divisor on $E$ with $d=\operatorname{deg} \mathbf{d}<\mu$. Let $R=T(\mathbf{d})$. Then:

(1) $R$ has Hilbert series $h_{R}(t)=h_{T}(t)-d /(1-t)^{3}$.

(2) $R$ is g-divisible with $R / g R=B(E, \mathcal{M}(-\mathbf{d}), \tau)$.

(3) $R$ is strongly noetherian, Auslander-Gorenstein, $C M$, and a maximal order. Further, $R$ satisfies the Artin-Zhang $\chi$ conditions, and has cohomological dimension 2 and a balanced dualizing complex.

(4) $R$ is generated in degree 1 if $\mu-d \geq 2$. If $\mu-d=1$, then $R_{m} R_{n}=R_{m+n}$ for all $m, n \geq 2$; in particular, $R$ is generated in degrees $\leq 2$, and the Veronese ring $R^{(2)}$ is generated in degree 1 .

(5) $T_{\leq \ell} * T(\mathbf{d})$ is a finitely generated right $R=T(\mathbf{d})$-module for each $\ell \geq 0$.

(6) If $\mu-d \geq 2$ then $T_{\leq \ell} * T(\mathbf{d})=T_{\leq \ell} T(\mathbf{d})$.

Proof. This summarises earlier results. In more detail, Parts $(1,2)$ follow from Proposition 5.2 with $\ell=0$; Part (3) is a consequence of Proposition 2.4; while Parts (4,5) are consequences of Proposition 4.9(2).

Since both sides of Part (6) are equal to $T_{n}$ in degree $n \leq \ell$, it is enough to show that $T_{\ell} M(n-\ell, \mathbf{d})_{n-\ell}=$ $M\left(n-\ell, \tau^{-\ell}(\mathbf{d})\right)_{n}$ for all $n \geq \ell$. Because $\operatorname{deg} \mathbf{d} \leq \mu-2$, this also follows from Proposition 4.9(2).

In [VB2] Van den Bergh only defines blowing up at a single point, and so it is useful to know that our multiple blowups can also be defined iteratively.

Proposition 5.4. Let $\mathbf{d}$ be a divisor on $E$ with $\operatorname{deg} \mathbf{d}<\mu$. Suppose that $\mathbf{d}=\mathbf{c}+\mathbf{e}$, where $\mathbf{c}$, e are effective, and let $R=T(\mathbf{c})$. Then $T(\mathbf{d})=R(\mathbf{e})=(T(\mathbf{c}))(\mathbf{e})$.

Before proving this, we give a lemma, which will be needed to handle the case $\operatorname{deg} \mathbf{d}=\mu-1$.

Lemma 5.5. Suppose that $\operatorname{deg} \mathbf{c}=\mu-2$ and write $R=T(\mathbf{c})$. Let $q \in E$. Define $V=\bigoplus V_{n} \subset T$ by

$$
\begin{gathered}
V_{n}=J_{T}\left(\mathbf{c}_{n}+q, \tau^{-1}\left(\mathbf{c}_{n-1}\right), \ldots, \tau^{-n+1}(\mathbf{c})\right)_{n} . \\
17
\end{gathered} .
$$


Then $V=J_{R}(q)$.

Proof. It follows by comparing divisor data that $V \subseteq R$. By Lemma 3.5(3),

$$
J_{R}(q)_{n}=\left\{x \in R_{n} \mid \bar{x} \in H^{0}\left(E, \mathcal{M}(-\mathbf{c})_{n}(-q)\right)\right\} .
$$

The top-layer vanishing of $V_{n}$ gives that $V \subseteq J_{R}(q)$. By Lemma 4.4(3), $V$ and $J_{R}(q)$ have the same Hilbert series, and thus the two are equal.

Proof of Proposition 5.4. In most cases, the proposition follows from the fact that the rings in question are generated in degree 1. Specifically, suppose that $\operatorname{deg} \mathbf{d} \leq \mu-2$. Then, by Theorem [5.3. $T(\mathbf{d})$ is generated in degree 1. Similarly, as $\operatorname{deg} \mathbf{e} \leq \operatorname{deg} \mathcal{M}(-\mathbf{c})-2=\mu-\operatorname{deg} \mathbf{c}-2$ the same argument implies that $R(\mathbf{e})$ is generated in degree 1. But now $T(\mathbf{d})_{1}=\left\{x \in T_{1} \mid \bar{x} \in H^{0}(E, \mathcal{M}(-\mathbf{d}))\right\}$ and, similarly, $R_{1}=T(\mathbf{c})_{1}=\left\{x \in T_{1} \mid \bar{x} \in H^{0}(E, \mathcal{M}(-\mathbf{c}))\right\}$. Then $R(\mathbf{e})_{1}=\left\{y \in R_{1} \mid \bar{y} \in H^{0}(E, \mathcal{M}(-\mathbf{c})(-\mathbf{e}))\right\}=T(\mathbf{d})_{1}$ and hence $R(\mathbf{e})=T(\mathbf{d})$.

Thus the only issue is when $\operatorname{deg} \mathbf{d}=\mu-1$, so assume that this is the case. A routine induction reduces to the case when $\operatorname{deg} \mathbf{e}=1$, so assume that $\mathbf{e}=p$ is a closed point on $E$. By Theorem [5.3, the rings $T(\mathbf{d})$ and $R(p)$ are generated in degrees 1 and 2 . They are equal in degree 1 by the argument from the first paragraph of the proof and so, in order to prove the proposition, it suffices to show that they are equal in degree 2.

Define a graded subspace $J=\bigoplus_{n \geq 0} J_{n}$ of $T$ by setting

$$
J_{n}=J_{T}\left(\mathbf{c}_{n}+p+\tau^{-1}(p), \tau^{-1}\left(\mathbf{c}_{n-1}+p\right), \tau^{-2}\left(\mathbf{c}_{n-2}\right), \ldots, \tau^{-n+1}\left(\mathbf{c}_{1}\right)\right)_{n},
$$

in the notation of Definition 4.1] It is immediate from the divisor data for the $J_{n}$ that $J \subseteq R$. Also, $J_{2}=T(\mathbf{d})_{2}$. As in the proof of Lemma 4.9(1), it follows from Lemmas 4.8(2) and 3.10 that $J$ is a right ideal of $R$. Let $J^{\prime}=M_{R}(2, p)=J_{R}\left(p+\tau^{-1}(p), \tau^{-1}(p)\right)$ which, by definition, is a saturated right ideal of $R$ with $J_{2}^{\prime}=R(p)_{2}$. Clearly $\tau^{k-1}(p) \notin \tau^{-2}(\mathbf{c})$ for $k \gg 0$, so choose some such $k \geq 2$. We next show that $J_{k}^{\prime}=J_{k}$.

We establish some notation. Let $V=J_{T}\left(\tau^{k-1}(p)+\mathbf{c}_{2}, \tau^{-1}(\mathbf{c})\right)_{2}$ and $I=J_{R}(p)$. We now work in $X=$ qgr- $T$, so let $\omega:$ Qgr- $T \rightarrow$ Gr- $T$ be the right adjoint to $\pi:$ Gr- $T \rightarrow$ Qgr- $T$. For an effective divisor $\mathbf{x}$ on $E$, let $G_{\mathbf{x}}$ be the functor on qgr- $T$ defined in Notation 3.8 Define $\mathcal{I}, \mathcal{K} \in X$ by

$$
\begin{aligned}
& \mathcal{I}=\mathcal{J}_{T}\left(p+\mathbf{c}_{k}, \tau^{-1}\left(\mathbf{c}_{k-1}\right), \ldots, \tau^{-k+1}(\mathbf{c})\right), \quad \text { and } \\
& \mathcal{K}=G_{\tau^{k-1}(p)} \circ G_{\tau^{-1}(\mathbf{c})} \circ G_{\mathbf{c}}\left(\mathcal{O}_{X}\right)=G_{\tau^{k-1}(p)} \mathcal{J}_{T}\left(\mathbf{c}_{2}, \tau^{-1}(\mathbf{c})\right),
\end{aligned}
$$

where the final equality follows from Lemma 3.10. By Lemma 5.5 we have $V=J_{R}\left(\tau^{k-1}(p)\right)_{2}$ and $(\omega \mathcal{I})_{k}=I_{k}$. We compute divisor data on $T$ for $\mathcal{K}$. By Lemma 3.9, $\mathcal{K}=\mathcal{J}_{T}\left(\mathbf{x}^{0}, \mathbf{x}^{1}, \mathbf{x}^{2}\right)$, where:

$$
\begin{aligned}
& \mathbf{x}^{0}=\tau^{k-1}(p)+\mathbf{c}+\tau^{-1}(\mathbf{c})=\tau^{k-1}(p)+\mathbf{c}_{2}, \\
& \mathbf{x}^{1}=\min \left(\tau^{k-1}(p)+\tau^{-1}(\mathbf{c}), \tau^{-1}(\mathbf{c})+\tau^{-2}(\mathbf{c})\right) \\
& \mathbf{x}^{2}=\min \left(\tau^{k-1}(p), \tau^{-2}(\mathbf{c})\right)
\end{aligned}
$$

Now, $\mathbf{x}^{2}=0$ by choice of $k$, and $\mathbf{x}^{\bullet}=\left(\mathbf{c}_{2}+\tau^{k-1}(p), \tau^{-1}(\mathbf{c})\right)$. Thus $V=(\omega \mathcal{K})_{2}$. 
Apply Lemma $4.8(2)$ to the product $I_{k} V=(\omega \mathcal{I})_{k}(\omega \mathcal{K})_{2}$ to get

$$
I_{k} V \subseteq W=\omega\left(\left[\tau^{-k}\left(G_{\tau^{k-1}(p)} \circ G_{\tau^{-1}(\mathbf{c})} \circ G_{\mathbf{c}}\right)\right](\mathcal{I})\right)_{k+2} .
$$

We use previous results to compute $W$. Let $\mathbf{w}^{\bullet}$ be the defining data for $\tau^{-k}\left(G_{\tau^{-1}(\mathbf{c})}{ }^{\circ} G_{\mathbf{c}}\right)(\mathcal{I})$. By Lemma 3.10 $\mathbf{w}^{0}=p+\mathbf{c}_{k+2}$. The formula for $\mathbf{w}^{i}$ for $i \geq 1$ is a bit more complicated. However, by Lemma 3.10 , $\mathbf{w}^{i} \leq \mathbf{z}^{i}+\tau^{-k}(\mathbf{c})+\tau^{-k-1}(\mathbf{c})$, where $\mathbf{z}^{\bullet}$ defines $\mathcal{I}$. From the formula for $\mathcal{I}$, we see that $\mathbf{w}^{i} \leq \tau^{-i}\left(\mathbf{c}_{k+2-i}\right)$. On the other hand,

$$
\tau^{-k}\left(G_{\tau^{-1}(\mathbf{c})} \circ G_{\mathbf{c}}\right)(\mathcal{I}) \subseteq G_{\tau^{-k-1}(\mathbf{c})} \circ G_{\tau^{-k}(\mathbf{c})} \mathcal{J}_{T}\left(\mathbf{c}_{k}, \tau^{-1}\left(\mathbf{c}_{k-1}\right), \ldots\right),
$$

and so $\mathbf{w}^{i} \geq \tau^{-i}\left(\mathbf{c}_{k+2-i}\right)$, by Lemma 3.10 once again. Thus $\mathbf{w}^{i}=\tau^{-i}\left(\mathbf{c}_{k+2-i}\right)$ for $i \geq 1$.

By Lemma $3.9 \tau^{-k}\left(G_{\tau^{k-1}(p)} \circ G_{\tau^{-1}(\mathbf{c})} \circ G_{\mathbf{c}}\right)(\mathcal{I})$ is then defined by $\mathbf{x}^{\bullet}$ where

$$
\mathbf{x}^{0}=p+\tau^{-1}(p)+\mathbf{c}_{k+2}, \quad \mathbf{x}^{1}=\tau^{-1}(p)+\tau^{-1}\left(\mathbf{c}_{k+1}\right),
$$

and for $i \geq 2$ we have

$$
\mathbf{x}^{i}=\min \left(\tau^{-i}\left(\mathbf{c}_{k+2-i}\right)+\tau^{-1}(p), \tau^{-i}\left(\mathbf{c}_{k+3-i}\right)\right) \geq \tau^{-i}\left(\mathbf{c}_{k+2-i}\right) .
$$

Thus

$$
W \subseteq J_{T}\left(p+\tau^{-1}(p)+\mathbf{c}_{k+2}, \tau^{-1}(p)+\tau^{-1}\left(\mathbf{c}_{k+1}\right), \tau^{-2}\left(\mathbf{c}_{k}\right), \ldots\right)_{k+2}=J_{k+2} .
$$

By Lemma 4.9(2), on the other hand, we have

$$
I_{k} V=J_{R}(p)_{k} J_{R}\left(\tau^{k-1}(p)\right)_{2}=M_{R}(1, p)_{k} M_{R}\left(1, \tau^{k-1}(p)\right)_{2}=M_{R}(2, p)_{k+2}=J_{k+2}^{\prime} .
$$

Thus $J_{k+2}^{\prime} \subseteq J_{k+2}$ by (5.6). However, by Lemma 4.4(3), twice, $\operatorname{dim}_{\mathbb{k}} J_{n}=\operatorname{dim}_{\mathbb{k}} R_{n}-3=\operatorname{dim}_{\mathbb{k}} J_{n}^{\prime}$ for $n \geq 2$. Thus $J_{k+2}^{\prime}=J_{k+2}$ for all $k \gg 0$.

Finally, let $\omega_{R}$ and $\pi_{R}$ denote the relevant functors on the module categories of $R$. As $J^{\prime}$ is a saturated right ideal of $R$, we have $J^{\prime}=\omega_{R} \pi_{R}\left(J^{\prime}\right)=\omega_{R} \pi_{R}(J) \supseteq J$. Comparing Hilbert series again, we see that $J_{n}^{\prime}=J_{n}$ in degrees $n \geq 2$. In particular,

$$
T(\mathbf{d})_{2}=J_{2}=J_{2}^{\prime}=R(p)_{2},
$$

which is what we needed to prove.

Remark 5.9. A subtle but important point is that, a priori, the construction of $J\left(\mathbf{d}^{\bullet}\right)$ and hence $T(\mathbf{d})$ depends upon a choice of the functors $(\widehat{-})_{p}$ in Theorem 3.1, these functors are not unique, and different functors may give different $J$ 's. However, the proof of Proposition 5.4 shows that $T(\mathbf{d})$ is uniquely determined by d. To see this note that, by Lemma 3.5. $M(1, \mathbf{d})=\bigoplus\left\{x \in T_{n} \mid \bar{x} \in H^{0}\left(E, \mathcal{M}_{n}(-\mathbf{d})\right)\right\}$ and hence $T(\mathbf{d})_{1}$ is clearly determined by $\mathbf{d}$. If $\operatorname{deg} \mathbf{d}<\mu-1$ then $T(\mathbf{d})$ is generated in degree 1 and so is determined by $\mathbf{d}$. So suppose that $\operatorname{deg} \mathbf{d}=\mu-1$, and write $\mathbf{d}=\mathbf{c}+p$ as in the above proof. Then (5.7) shows that $M_{R}(2, p)_{k+2}$ for $k \gg 0$ is determined by the appropriate modules $M\left(1, \tau^{j}(p)\right)$. Hence the saturation $M_{R}(2, p)$, and thus by (5.8) $T(\mathbf{d})_{2}=T(\mathbf{c})(p)_{2}=M_{R}(2, p)_{2}$, is determined by $\mathbf{c}$ and $p$. This last equation holds for any choice of $\mathbf{c}$ and 
$p$, and so $T(\mathbf{d})_{2}$ is determined by $\mathbf{d}$. Since $T(\mathbf{d})$ is generated in degrees 1 and 2 , it is uniquely determined by $\mathbf{d}$.

This is also useful in a second case, when one takes an algebraically closed field extension $\mathbb{k} \subset K$. In this case, given a divisor $\mathbf{d}$ on $E_{\mathrm{k}}$ we can either form $T(\mathbf{d}) \otimes_{\mathbb{k}} K$ or regard $\mathbf{d}$ as a divisor on $E \otimes_{\mathbb{k}} K$ and form $\left(T \otimes_{\mathbb{k}} K\right)(\mathbf{d})$. The potential difference between these two constructions lies in the fact that one is using two distinct versions of $(\widehat{-})_{p}$ : either defined directly on $C_{p}(K)$ or by taking the functors from $C_{p}(\mathbb{k})$ and then tensoring up with $K$. It is easy to see that $M_{T \otimes K}(1, \mathbf{d})=M_{T}(1, \mathbf{d}) \otimes_{\mathbb{k}} K$, and a similar argument to the previous paragraph shows that $T(\mathbf{d}) \otimes_{\mathbb{k}} K=\left(T \otimes_{\mathbb{k}} K\right)(\mathbf{d})$.

To end the section, we use the earlier results to construct right $T(\mathbf{d})$-modules corresponding to certain very particular kinds of divisor data. These will be used in the companion paper [RSS to give explicit examples of interesting endomorphism rings of modules over $T(\mathbf{d})$, known as virtual blowups.

For a divisor $\mathbf{z} \in \operatorname{Div} E$, write $\mathbf{z}=\sum z_{q} q$ and let $\mathbf{z}_{+}=\max (\mathbf{z}, 0)=\sum_{\left\{q \mid z_{q} \geq 0\right\}} z_{q} q$ be the minimal effective divisor greater than or equal to $\mathbf{z}$.

Lemma 5.10. Let $\mathbf{d}$ be an effective divisor on $E$ so that $\operatorname{deg} \mathbf{d}<\mu$. Let $\mathbf{y}$ be a divisor on $E$ so that $0 \leq$ $\mathbf{y} \leq \mathbf{d}_{k}$ for some $k$. Then there is a g-divisible finitely generated right $T(\mathbf{d})$-module $M$ with $T(\mathbf{d}) \subseteq M \subseteq T$ so that $\bar{M} \doteq \bigoplus_{n} H^{0}\left(E, \mathcal{M}_{n}\left(-\mathbf{d}_{n}+\mathbf{y}\right)\right)$.

Proof. If $0 \leq i \leq m-1$, let $\mathbf{z}^{m, i}=\tau^{-i}\left(\mathbf{d}_{m-i}-\mathbf{y}\right)_{+}$. Note that, if $\mathbf{z} \leq \mathbf{z}^{\prime} \in \operatorname{Div}(E)$, then $\mathbf{z}_{+} \leq \mathbf{z}_{+}^{\prime}$. Thus, for fixed $m$, it follows that $\mathbf{z}^{m, \bullet}$ is an allowable divisor sequence. For $m \in \mathbb{N}$, define $M_{m}=J\left(\mathbf{z}^{m, \bullet}\right)_{m}$; thus $M=\bigoplus_{m \geq 0} M_{m} \subseteq T$. Since $\mathbf{z}^{m+1, i+1}=\tau^{-1}\left(\mathbf{z}^{m, i}\right)$, using Lemma 4.4 (2) one graded piece at a time we see that $M \cap T g=M g$. It is easy to see that $M \supseteq T(\mathbf{d})$ by comparing the divisor sequence $\mathbf{z}^{m, \bullet}$ for $M_{m}$ with the divisor sequence $\left(\tau^{-i}\left(\mathbf{d}_{m-i}\right)\right)_{i}$ for $T(\mathbf{d})_{m}$.

It remains to show that $M$ is a right $T(\mathbf{d})$-module, for which we use Lemma 4.8(2). Fix $n, m \in \mathbb{Z}_{\geq 1}$. Let $\mathcal{F}:=\mathcal{J}\left(\mathbf{z}^{m, \bullet}\right)$, so $M_{m}=\omega(\mathcal{F})_{m}$, and let $\mathcal{F}^{\prime}:=\mathcal{J}\left(\mathbf{z}^{n+m, \bullet}\right)$. By Lemma 4.9. $T(\mathbf{d})_{n}$ is the degree $n$ part of $\omega\left(\tau^{-(n-1)} G_{\mathbf{d}} \circ \cdots \circ G_{\mathbf{d}}\left(\mathcal{O}_{X}\right)\right)$, where $G_{\mathbf{d}}$ is the functor defined in Notation 3.8. Thus, by Lemma 4.8 .

$$
M_{m} T(\mathbf{d})_{n} \subseteq\left(\omega\left(\tau^{-(m+n-1)} G_{\mathbf{d}} \circ \cdots \circ \tau^{-m} G_{\mathbf{d}}(\mathcal{F})\right)\right)_{m+n} .
$$

To show that $M$ is a right $T(\mathbf{d})$-module, it therefore suffices to show that

$$
\tau^{-(m+n-1)} G_{\mathbf{d}} \circ \cdots \circ \tau^{-m} G_{\mathbf{d}}(\mathcal{F}) \subseteq \mathcal{F}^{\prime}
$$

By Lemma 3.10, $\tau^{-(m+n-1)} G_{\mathbf{d}} \circ \cdots \circ \tau^{-m} G_{\mathbf{d}}(\mathcal{F})$ is defined by $\mathbf{w}^{\bullet}$, where

$$
\mathbf{w}^{i}=\min _{j=0}^{i}\left(\tau^{-j}\left(\mathbf{z}^{m, i-j}\right)+\tau^{-m-j}\left(\mathbf{d}_{n-j}\right)\right) .
$$

To show (5.11), we must show that $\mathbf{w}^{i} \geq \mathbf{z}^{n+m, i}$. It therefore suffices to show for all $0 \leq j \leq i$ that

$$
\mathbf{r}=\tau^{-j}\left(\mathbf{z}^{m, i-j}\right)+\tau^{-m-j}\left(\mathbf{d}_{n-j}\right) \geq \mathbf{z}^{n+m, i} .
$$


Now,

$$
\mathbf{r} \geq \tau^{-i}\left(\mathbf{d}_{m+j-i}-\mathbf{y}+\tau^{-m-j+i}\left(\mathbf{d}_{n-j}\right)\right)=\tau^{-i}\left(\mathbf{d}_{n+m-i}-\mathbf{y}\right) .
$$

Since $\mathbf{r}$ is effective, $\mathbf{r} \geq \max \left(0, \tau^{-i}\left(\mathbf{d}_{n+m-i}-\mathbf{y}\right)\right)=\mathbf{z}^{n+m, i}$. Thus (5.12) holds.

Finally, since $\operatorname{deg} \mathbf{z}^{n, i} \leq \operatorname{deg} \mathbf{d}_{n-i}=(n-i)(\operatorname{deg} \mathbf{d})<(n-i) \mu$, Lemma 4.4(3) implies that $\bar{M}_{n}=$ $H^{0}\left(E, \mathcal{M}_{n}\left(-\mathbf{z}^{n, 0}\right)\right)_{n}$ for each $n \geq 0$. By assumption $\mathbf{y} \leq \mathbf{d}_{k} \leq \mathbf{d}_{n}$ for all $n \geq k$, and so it follows that $\mathbf{z}^{n, 0}=\left(\mathbf{d}_{n}-\mathbf{y}\right)_{+}=\left(\mathbf{d}_{n}-\mathbf{y}\right)$ for all $n \geq k$, and hence $\bar{M}_{\geq k}=\bigoplus_{n \geq k} H^{0}\left(E, \mathcal{M}_{n}\left(-\mathbf{d}_{n}+\mathbf{y}\right)\right)$.

\section{Relating LeFt And Right ideals}

The main assumption of this paper, Hypothesis 2.2, is left-right symmetric and so one can define left ideals of $T$ analogous to the $J\left(\mathbf{d}^{\bullet}\right)$, with similar properties. What is less obvious is that these left ideals are closely related to the corresponding right ideals. In this section we study this relationship. This will be useful for the study of two-sided ideals of $T$, which will be the theme of the last two sections of this paper.

Throughout the section, let $T$ be a ring satisfying Hypothesis 2.2 with $B=T / g T \cong B(E, \mathcal{M}, \tau)$. Recall that the equivalence of categories $\operatorname{coh} E \rightarrow$ qgr- $B$ is given by $\mathcal{F} \mapsto \bigoplus_{n \geq 0} H^{0}\left(E, \mathcal{F} \otimes \mathcal{M}_{n}\right)$ while the equivalence $\operatorname{coh} E \rightarrow B$-qgr is given by $\mathcal{F} \mapsto \pi\left(\bigoplus_{n \geq 0} H^{0}\left(E, \mathcal{M}_{n} \otimes \mathcal{F}^{\tau^{n-1}}\right)\right)$. For $q \in E$, write

$$
\mathcal{O}_{q}^{\prime}:=\pi\left(\bigoplus_{n \geq 0} H^{0}\left(E, \mathcal{M}_{n} \otimes \mathbb{k}(q)^{\tau^{n-1}}\right)\right)
$$

for the object in $B$-qgr (or $T$-qgr) corresponding to the skyscraper sheaf $\mathbb{k}(q)$ under the latter equivalence. There is a left-sided version of Theorem 3.1 giving an exact functor $\widetilde{(-)_{p}}: T$-qgr $\rightarrow\left(C_{p}\right.$-mod).

Typically, if $\tau^{-n}$ appears in some right-sided formula, then $\tau^{n}$ appears in the left-sided analogue. Thus, for example, we define a left allowable divisor layering to be a sequence of divisors $\mathbf{d}^{\bullet}=\left(\mathbf{d}^{0}, \mathbf{d}^{1}, \ldots, \mathbf{d}^{k-1}\right)$ on $E$ that satisfies $\tau\left(\mathbf{d}^{i-1}\right) \geq \mathbf{d}^{i}$ for $1 \leq i \leq k-1$. For such a layering $\mathbf{d}^{\bullet}$, we define a saturated left ideal $J^{\prime}\left(\mathbf{d}^{\bullet}\right)=J_{T}^{\prime}\left(\mathbf{d}^{\bullet}\right)$ of $T$ in a way analogous to Definition 3.4. In more detail, if $\mathbf{d}^{\bullet}$ is supported on $\mathbb{O}(p)$, say with $\mathbf{d}^{i}=\sum_{j} a_{j, j-i} \tau^{j}(p)$ define $J^{\prime}$ to be the saturated left ideal of $T$ for which $\widetilde{\pi\left(J^{\prime}\right)_{p}}$ is the left ideal of $C_{p}$ with $(x)^{a_{k, \ell}}$ in the $(k, \ell)$-spot. In general $J^{\prime}\left(\mathbf{d}^{\bullet}\right)$ is defined as before to be the intersection of the left ideals obtained by restricting the divisor layering to each relevant $\tau$-orbit.

The left-sided analogue of Lemma 3.5, with essentially the same proof, is as follows.

Lemma 6.1. Let $\mathbf{d}^{\bullet}$ be a left allowable divisor layering and let $J^{\prime}=J^{\prime}\left(\mathbf{d}^{\bullet}\right)$ and $M=T / J^{\prime}$.

(1) If $M^{j}=M g^{j} / M g^{j+1}$ is the $j^{\text {th }}$ layer of $M$, then in B-qgr we have

$$
\pi\left(M^{j}\right) \cong \pi\left(\bigoplus_{n \geq 0} H^{0}\left(E, \mathcal{M}_{n} \otimes\left(\mathcal{O}_{E} / \mathcal{O}_{E}\left(-\tau^{-n+1}\left(\mathbf{d}^{j}\right)\right)\right)\right)\right) .
$$

(2) $\left(\overline{J^{\prime}}\right)^{\text {sat }}=\bigoplus_{n \geq 0} H^{0}\left(E, \mathcal{M}_{n}\left(-\tau^{-n+1}\left(\mathbf{d}^{0}\right)\right)\right)$.

(3) If $\mathbf{d}^{\bullet}=(\mathbf{d})$ has length 1 , then $J^{\prime}(\mathbf{d})=\bigoplus_{n \geq 0}\left\{x \in T_{n} \mid \bar{x} \in H^{0}\left(E, \mathcal{M}_{n}\left(-\tau^{-n+1}(\mathbf{d})\right)\right)\right\}$.

We also have left-sided versions of Definition 4.1 and Lemma 4.9 
Definition 6.2. For any divisor d, define $M^{\prime}(k, \mathbf{d})=M_{T}^{\prime}(k, \mathbf{d})=J^{\prime}\left(\mathbf{d}^{\bullet}\right)$ for the divisor layering

$$
\mathbf{d}^{0}=\mathbf{d}+\tau(\mathbf{d})+\cdots+\tau^{k-1}(\mathbf{d}), \mathbf{d}^{1}=\tau(\mathbf{d})+\cdots+\tau^{k-1}(\mathbf{d}), \ldots, \mathbf{d}^{k-1}=\tau^{k-1}(\mathbf{d}) .
$$

Lemma 6.3. Let $n, m, k, l \in \mathbb{N}$. Then

$$
M^{\prime}\left(\ell, \tau^{k-m}(\mathbf{d})\right)_{n} M^{\prime}(k, \mathbf{d})_{m} \subseteq M^{\prime}(k+\ell, \mathbf{d})_{n+m}^{\prime} .
$$

If $\mu-\operatorname{deg} \mathbf{d} \geq 2$, this is an equality for all $m \geq k$ and $n \geq \ell$. If $\mu-\operatorname{deg} \mathbf{d}=1$, it is an equality for all $m \geq \max (2, k)$ and $n \geq \max (2, \ell)$.

For the rest of the section we consider another family of right ideals defined by divisor data, together with their left-sided versions, which will be useful in [RSS]. We write $p_{i}=\tau^{-i}(p)$ for a closed point $p \in E$.

Definition 6.4. Let $i \in \mathbb{N}$ and let $0 \leq r \leq d \leq \mu \in \mathbb{N}$. For any fixed point $p \in E$, define $Q(i, r, d, p)$ to be the right ideal $J\left(\mathbf{d}^{\bullet}\right)$ of $T$ associated to the divisor layering:

$$
\mathbf{d}^{0}=d p+d p_{1}+\cdots+d p_{i-1}, \mathbf{d}^{1}=d p_{1}+\cdots+d p_{i-1}, \ldots, \mathbf{d}^{i-1}=r p_{i-1} .
$$

Analogously, define $Q^{\prime}(i, r, d, p)$ to be the left ideal $J^{\prime}\left(\mathbf{d}^{\bullet}\right)$ of $T$ associated to the left divisor layering:

$$
\mathbf{d}^{0}=d p+d p_{-1}+\cdots+d p_{-i+1}, \mathbf{d}^{1}=d p_{-1}+\cdots+d p_{-i+1}, \ldots, \mathbf{d}^{i-1}=r p_{-i+1} .
$$

Note that the divisor data for $Q(i, d, r, p)$ equals that for $M(i, d p)$, except that $\mathbf{d}^{i-1}$ may have smaller multiplicity.

For $p \in E$, recall that the right point module corresponding to the point $p$ is written $P(p)=T / J(p)$, with $\pi(P(p))=\mathcal{O}_{p}$ in qgr- $T$. Similarly, write $P^{\prime}(p)=T / J^{\prime}(p)$ for the left point module corresponding to $p$.

Lemma 6.5. Let $i, r, d, n \in \mathbb{N}$, with $i<n$ and $1 \leq r \leq d \leq \mu$, and $p \in E$.

(1) $Q(i, r, d, p) \subseteq Q(i, r-1, d, p)$, with factor $[Q(i, r-1, d, p) / Q(i, r, d, p)]_{\geq n} \cong P\left(p_{i-n-1}\right)[-n]$.

(2) Similarly, $Q^{\prime}(i, r, d, p) \subseteq Q^{\prime}(i, r-1, d, p)$ with

$$
\left[Q^{\prime}(i, r-1, d, p) / Q^{\prime}(i, r, d, p)\right]_{\geq n} \cong P^{\prime}\left(p_{-i+n+1}\right)[-n]
$$

Proof. We prove only Part (1). By Lemma 3.5, the image of the module $W=\frac{Q(i, r-1, e, p)}{Q(i, r, e, p)}$ in $X=$ qgr- $T$ is a copy of $\mathcal{O}_{p_{i-1}}$. This simple object is also equal to $\pi\left(P\left(p_{i-n-1}\right)[-n]\right)$, so the required isomorphism holds in large degree. Now use Lemma 4.4 to see that $W_{\geq n}$ has the same Hilbert series as $P\left(p_{i-n-1}\right)[-n]$. Since each $Q$ is saturated, $W$ is torsion-free and so we do have an isomorphism in the claimed degrees.

Lemma 6.6. Let $n>\ell \in \mathbb{N}$ and let $\mathbf{d}=\sum e_{p} p$ be a nonzero effective divisor on $E$ of degree at most $\mu-1$, supported at points with distinct $\tau$-orbits. Then

$$
\left(T_{\leq \ell} * T(\mathbf{d})\right)_{n}=\bigcap\left\{Q\left(i, r, e_{p}, p_{j}\right)_{n} \mid p \in \mathbf{d}, i \geq 1, \ell \leq j \leq n-i, 1 \leq r \leq e_{p}\right\} .
$$

In addition, $T_{\leq \ell} * T(\mathbf{d})=\bigcap_{p} T_{\leq \ell} * T\left(e_{p} p\right)$. 
Proof. To prove the first assertion it is enough to consider the case when $\mathbf{d}=e_{p} p$ is a single multiple point. This is an easy consequence of Lemma 3.6. The final statement is immediate from the definitions.

For any $i \geq 2, e \geq 1$, and $q \in E$, the following identity also follows immediately from Lemma 3.6

$$
Q(i, 0, e, q)=Q(i-1, e, e, q) \cap Q\left(i-1, e, e, \tau^{-1}(q)\right) .
$$

We are now ready to prove the main result of this section, relating the right and left ideals defined above.

Proposition 6.8. Fix an effective divisor $\mathbf{d}$ on $E, n, k, r, m \in \mathbb{N}$ with $r \leq m$, and $p \in E$.

(1) $\overline{M(1, \mathbf{d})}_{n}=H^{0}\left(E, \mathcal{M}_{n}(-\mathbf{d})\right)_{n}={\overline{M^{\prime}\left(1, \tau^{n-1}(\mathbf{d})\right)_{n}}}_{n}$.

(2) $M(k, \mathbf{d})_{n}=M^{\prime}\left(k, \tau^{n-k}(\mathbf{d})\right)_{n}$.

(3) $Q(k, r, m, p)_{n}=Q^{\prime}\left(k, r, m, \tau^{n-k}(p)\right)_{n}$.

Proof. (1) This follows immediately from Lemmas 3.5(3) and 6.1(3).

(2) When $k=0$, the result is trivial. We first prove by induction that, for each $k \geq 1$, (2) holds if $n \gg 0$. Assume that $k=1$. Since both $M(1, \mathbf{d})$ and $M^{\prime}\left(1, \tau^{n-1}(\mathbf{d})\right)$ contain $g T$ (use Lemmas 3.5(3) and 6.1(3)), it

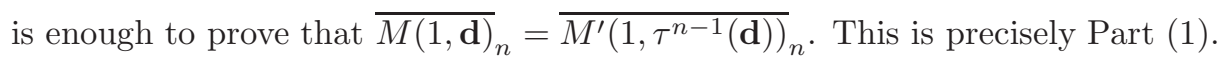

Now for $k \geq 2$, we observe that

$$
\begin{aligned}
M(k, \mathbf{d})_{n+r}= & M(k-1, \mathbf{d})_{r} M\left(1, \tau^{r-k+1}(\mathbf{d})\right)_{n}=M^{\prime}\left(k-1, \tau^{r-k+1}(\mathbf{d})\right)_{r} M^{\prime}\left(1, \tau^{n+r-k}(\mathbf{d})\right)_{n} \\
& =M^{\prime}\left(k, \tau^{n+r-k}(\mathbf{d})\right)_{n+r},
\end{aligned}
$$

for all $n, r \gg 0$. Here, the first equality follows from Lemma 4.9 the second from the induction hypothesis, and the last from Lemma 6.3. This proves the induction step, and so (2) holds for each $k$, provided $n \gg 0$.

To get the result for all $n$, note first that for fixed $n \geq 0$, we have for all $\ell \gg 0$ that

$$
T_{\ell} M(k, \mathbf{d})_{n} \subseteq M\left(k, \tau^{-\ell}(\mathbf{d})\right)_{n+\ell}=M^{\prime}\left(k, \tau^{n-k}(\mathbf{d})\right)_{n+\ell},
$$

where the first inclusion is a consequence of Lemma 4.9, and the equality holds since we have proven Part (2) for large $n+\ell$. Each $M^{\prime}$ is saturated by definition, and since (6.9) holds for all $\ell \gg 0$ we get $M(k, \mathbf{d})_{n} \subseteq M^{\prime}\left(k, \tau^{n-k}(\mathbf{d})\right)_{n}$. An analogous argument using Lemma 6.3 gives the reverse inclusion.

(3) It is easy to prove using Lemma 3.6 that

$$
Q(k, r, m, p)_{n}=M(k, r p)_{n} \cap M(k-1, m p)_{n} \cap M\left(k-1, m \tau^{-1}(p)\right)_{n} .
$$

Similarly, a left-sided version of that lemma shows that

$$
Q^{\prime}(k, r, m, q)_{n}=M^{\prime}(k, r q)_{n} \cap M^{\prime}(k-1, m q)_{n} \cap M^{\prime}(k-1, m \tau(q))_{n}
$$

for any point $q$. Now the result follows from Part (2) by taking $q=\tau^{n-k}(p)$.

One consequence of Proposition 6.8 is that $T(\mathbf{d})$ can be defined either using either left or right ideals:

$$
T(\mathbf{d})=\bigoplus_{n \geq 0} M(n, \mathbf{d})_{n}=\bigoplus_{n \geq 0} M^{\prime}(n, \mathbf{d})_{n}
$$




\section{Divisors AND EXCEPTIONAL LINE MODUlES}

As always, assume that $T$ is an algebra satisfying Hypothesis 2.2 The main goal of this section is to construct the exceptional line module for a one-point blowup $T(p) \subseteq T$, thereby proving Proposition 1.2 from the introduction. This will be used in the next section to understand the ideal structure of more general blowups $T(\mathbf{d})$ (see Theorem 8.8, in particular). The key tool in the proof will be the notion of the divisor associated to a $T$-module and so the first part of this section will concentrate on this concept.

In fact it will be useful to work more generally than Hypothesis 2.2. Thus to begin the section we fix the following notation. Let $S$ be a cg $\mathbb{k}$-algebra which is a domain with a central element $g \in S_{\gamma}$ for some $\gamma \geq 1$, such that $S / g S \cong B(E, \mathcal{L}, \sigma)$ for some elliptic curve $E$, invertible sheaf $\mathcal{L}$ with $\operatorname{deg} \mathcal{L} \geq 1$, and infinite order automorphism $\sigma$. Such a ring $S$ will be called Sklyanin-like, since the obvious examples are indeed the quadratic and cubic Sklyanin algebras of dimension three. Note that the Veronese $\operatorname{ring} T=S^{(\gamma)}$ satisfies Hypothesis 2.2 with $\mathcal{M}=\mathcal{L} \otimes \mathcal{L}^{\sigma} \otimes \cdots \otimes \mathcal{L}^{\sigma^{\gamma-1}}$ and $\tau=\sigma^{\gamma}$.

We now turn to the divisor of a module. This concept is extensively studied in [Ro], to which the reader is referred for more details.

Definition 7.1. Let $B=B(E, \mathcal{L}, \sigma)$ be as above with quotient map $\pi:$ gr- $B \rightarrow$ qgr- $B$. If $M \in$ gr- $B$ with GKdim $M=1$, then $\pi(M)$ corresponds to a torsion sheaf in $\operatorname{coh} E \sim$ qgr- $B$, which has a finite filtration by skyscraper sheaves $\mathbb{k}(q)$ for points $q \in E$. Write $\mathbf{c}(M)=\mathbf{c}_{B}(M)$ for the divisor $\sum c_{p} p$, where $c_{p}$ is the number of times $\mathbb{k}(p)$ occurs in this filtration.

Definition 7.2. Let Div $E$ denote the free abelian group generated by the closed points of the elliptic curve $E$ and set Pic $E=\operatorname{Div} E / \Lambda$, where $\Lambda$ is the subgroup of divisors linearly equivalent to 0 . It will be convenient to identify points of $E$ on the same $\sigma$-orbit, so let $\overline{\operatorname{Div}} E=\operatorname{Div} E / \Gamma$ where $\Gamma$ is the subgroup generated by $\{p-\sigma(p): p \in E\}$. Similarly, let $\overline{\operatorname{Pic}}(E)=\operatorname{Div} E /(\Gamma+\Lambda)$. Since $\sigma$ has infinite order, $\sigma(x)=x+\alpha$ is translation by some $\alpha \in E$ under the group law on $E$. It follows easily that $\overline{\operatorname{Pic}}(E)=\operatorname{Pic}(E) / \mathbb{Z} \alpha$.

Let $S$ be Sklyanin-like. Then $M \in \operatorname{gr}-S$ is called admissible if GKdim $M / M g \leq 1$. In this case the divisor associated to $M$ is

$$
\operatorname{Div} M=\operatorname{Div}_{S} M=\mathbf{c}(M / M g)-\mathbf{c}\left(\operatorname{Tor}_{S}^{1}(M, S / S g)\right) \in \operatorname{Div} E .
$$

For an admissible module $M$, let $\overline{\operatorname{Div}}(M)$ be the image of $\operatorname{Div} M$ in $\overline{\operatorname{Pic}}(E)$. Finally, let

$$
\operatorname{Div}(S)=\{\operatorname{Div} M \in \operatorname{Pic} E \mid M \text { is admissible }\}
$$

and let $\overline{\operatorname{Div}}(S)$ denote the image of $\operatorname{Div}(S)$ in $\overline{\operatorname{Pic}}(E)$.

There are analogous concepts for left $S$-modules. If $M \in S$-gr is an admissible left module we write its divisor as $\operatorname{Div}^{\ell}(M)$, with image $\overline{\operatorname{Div}}^{\ell}(M) \in \overline{\operatorname{Pic}}(E)$. Similarly, set

$$
\operatorname{Div}^{\ell}(S)=\left\{\operatorname{Div}^{\ell}(M) \mid M \in S \text {-gr is admissible }\right\} \quad \text { and } \quad \overline{\operatorname{Div}}^{\ell}(S)=\left\{\overline{\operatorname{Div}}^{\ell}(M) \mid M \in S \text {-gr is admissible }\right\} \text {. }
$$


A number of properties of Div $M$, mostly coming from [ $\underline{\mathrm{Ro}}$, are collected in the next lemma. In $[\mathrm{Ro}$, the assumption is made that $S / g S \cong B(E, \mathcal{L}, \sigma)$ where $\operatorname{deg} \mathcal{L} \geq 2$, but we wish to allow $\operatorname{deg} \mathcal{L}=1$. In most cases this makes no real difference and we can simply quote the result from [Ro]. In a few cases we need to give a different proof which also covers the case $\operatorname{deg} \mathcal{L}=1$.

Lemma 7.3. Let $S$ be Sklyanin-like.

(1) $M \in \operatorname{gr}-S$ is admissible precisely when $\operatorname{GKdim} M \leq 2$ and $\operatorname{GKdim}_{\operatorname{tors}}(M) \leq 1$.

(2) $\operatorname{Tor}_{S}^{1}(M, S / S g) \cong\{m \in M \mid m g=0\}[-\gamma]$ as graded $S$-modules. Thus, if $M$ is admissible and $g$-torsionfree then $\operatorname{Div}(M)$ is effective. If, in addition, $\operatorname{GKdim} M=2$, then $\operatorname{Div}(M) \neq 0$.

(3) $\operatorname{Div} M[n]=\sigma^{n}(\operatorname{Div} M)$ for any $n \in \mathbb{Z}$.

(4) If $0 \rightarrow M \rightarrow N \rightarrow P \rightarrow 0$ is an exact sequence of admissible $S$-modules, then $\operatorname{Div} N=\operatorname{Div} M+\operatorname{Div} P$.

(5) $\overline{\operatorname{Div}}(M)=0$ if $M \in \operatorname{gr}-S$ is admissible with $\operatorname{GKdim}(M)=1$.

(6) $\overline{\operatorname{Div}}(S)=\{\overline{\operatorname{Div}}(M) \mid M \in \operatorname{gr}-S$ is g-torsionfree and $\operatorname{GKdim}(M)=2\} \cup\{0\}$.

Proof. Parts (1-4) follow from [Ro, (8.1) and Lemma 8.3]. Part (5) is [Ro, Lemma 8.7]. For Part (6), suppose that $M \in \operatorname{gr}-S$ is admissible. Then $\operatorname{GKdim} M \leq 2$. If $N$ is the largest submodule of $M$ with $\operatorname{GKdim} N=1$, then $M / N$ is either 0 or GK 2-pure. If $M / N=0$ we are done, so assume $M / N$ is GK 2-pure. Now $\overline{\operatorname{Div}}(M / N)=\overline{\operatorname{Div}}(M)$ by Parts (4) and (5). Now $M / N$ is also admissible and so $\operatorname{tors}_{g}(M / N)=0$ by Part (1); thus $M / N$ has GK-dimension 2 and is $g$-torsionfree. Part (6) follows.

We now return to the setting of the rings $T$ satisfying Hypothesis 2.2, so that $g \in T_{1}$.

Lemma 7.4. Consider $R=T(\mathbf{d}+p) \subseteq \widetilde{R}=T(\mathbf{d})$, where $p \in E$ and $\mathbf{d}$ is effective with $\operatorname{deg} \mathbf{d}<\mu-1$. If $M \in \operatorname{gr}-\widetilde{R}$ is admissible, then $M$ is also admissible as a right $R$-module and $\operatorname{Div}_{R} M=\operatorname{Div}_{\widetilde{R}} M$.

Proof. Write $R / g R=B=B(E, \mathcal{N}, \tau) \subseteq \widetilde{R} / g \widetilde{R}=\widetilde{B}=B(E, \mathcal{M}, \tau)$, where $\mathcal{N}=\mathcal{M}(-p)$; thus $\operatorname{deg} \mathcal{N} \geq 1$. The key point is to show that, if $N \in \operatorname{gr}-\widetilde{B}$, then $N \in \operatorname{gr}-B$ with $\mathbf{c}_{B}(N)=\mathbf{c}_{\widetilde{B}}(N)$ (this is the analogue of [Ro, Lemma 3.5]). By [MR, (6.2.19) and (6.8.25)], $N$ has a composition series where the factors are either finite dimensional or 1-critical $\widetilde{R}$-modules. By a routine induction, we can therefore assume that $N$ is a cyclic 1-critical right $\widetilde{B}$-module. As such, Lemma $2.8(3)$ implies that (up to a shift) $N$ is a $\widetilde{B}$-point module: $N=\widetilde{B} / I$ where $I=\bigoplus_{n \geq 0} H^{0}\left(E, \mathcal{I}_{q} \otimes \mathcal{M}_{n}\right)$ and $\mathcal{I}_{q}$ is the ideal sheaf defining $q$.

Suppose that $\widetilde{B}_{n} B_{m} \subseteq I$ for some $n, m \gg 0$. Then $H^{0}\left(E, \mathcal{M}_{n}\right) H^{0}\left(E, \mathcal{N}_{m}^{\tau^{n}}\right) \subseteq H^{0}\left(E, \mathcal{I}_{q} \otimes \mathcal{M}_{n+m}\right)$. Choosing $n, m$ large enough so that all sheaves in this equation are generated by their sections, gives $\mathcal{M}_{n} \mathcal{N}_{m}^{\tau^{n}} \subseteq \mathcal{I}_{q} \otimes \mathcal{M}_{n+m}$ and hence $\mathcal{N}_{m}^{\tau^{n}} \subseteq \mathcal{I}_{q} \otimes \mathcal{M}_{m}^{\tau^{n}}$. But $\mathcal{M}_{m} / \mathcal{N}_{m}$ is supported at finitely many points. Thus, for $m \gg 0$, the equation $\mathcal{N}_{m}^{\tau^{n}} \subseteq \mathcal{I}_{q} \otimes \mathcal{M}_{m}^{\tau^{n}}$ only holds for finitely many $n \geq 0$. Thus for fixed $m \gg 0$, one has $\widetilde{B}_{n} B_{m} \not I I$ and hence $N_{n} B_{m}=N_{m+n}$ for all $n \gg 0$. Thus $N_{B}$ is finitely generated.

Let $Q(p)=\bigoplus_{n \geq 0} H^{0}\left(E, \mathbb{k}(p) \otimes \mathcal{N}_{n}\right)$ be the $B$-point module corresponding to a point $p \in E$. A similar calculation shows that $\left\{x \in B_{m} \mid N_{n} x=0\right\}=H^{0}\left(E,\left(\mathcal{I}_{q}\right)^{\tau^{-n}} \otimes \mathcal{N}_{m}\right)$ as long as $m, n \gg 0$. Thus for some 
$n \gg 0$ we have $N_{n} B \cong Q\left(\tau^{n}(q)\right)[-n]$ as $B$-modules. Hence $\pi(N)=\pi\left(Q\left(\tau^{n}(q)\right)[-n]\right)=\pi(Q(q))$ in qgr- $B$ and so $\mathbf{c}_{B}(N)=q$ as well.

This proves the analogue of [R0, Lemma 3.5] and now the proof of [R0, Proposition 9.5(1)] may be used to prove the present result.

Definition 7.5. A graded right $R$-module $M$ is called a line module if $M$ is finitely generated and has Hilbert series $h_{M}(t)=1 /(1-t)^{2}$.

As with point modules, line modules are often assumed to be cyclic, but this is not really appropriate when $T$ is not generated in degree one.

Lemma 7.6. Let $R=T(\mathbf{d})$ for some effective divisor $\mathbf{d}$ with $\operatorname{deg} \mathbf{d}<\mu$.

(1) Let $M$ be a g-torsionfree, admissible right $R$-module, with $\operatorname{GKdim} M=2$ such that $\operatorname{Div} M=p$ is a single point. Then $M$ is 2-critical.

(2) If $M$ is a g-torsionfree line module, then Part (1) applies and $M$ is 2-critical.

Remark 7.7. If $\mu-\mathbf{d} \geq 2$ then any cyclic line module for $T(\mathbf{d})$ is $g$-torsionfree, by [Ro, Lemma 8.9].

Proof. (1) By Lemma 7.3(1), $M$ is 2-pure. Thus, if $M$ is not critical, then it has a graded submodule $N$ with $\operatorname{GKdim} N=2$ such that $M / N$ is 2 -critical. Since $M / N$ is still admissible, Lemma 7.3(1) implies that $M / N$ is $g$-torsionfree. Then both $\operatorname{Div} N$ and $\operatorname{Div} M / N$ are nonzero effective divisors and $p=\operatorname{Div} M=$ Div $N+\operatorname{Div} M / N$ by Lemma 7.3) $(2,4)$. This is obviously impossible.

(2) Since $M$ is $g$-torsionfree, $M / M g$ has the Hilbert series $1 /(1-t)$, and so $P=M / M g$ is a point module. Thus $M$ is admissible. By Lemma $2.8(1,2), P_{\geq n} \cong P(q)_{\geq n}$ for some point module $P(q)$, and so $\pi(M)=\pi(P(q))=\mathcal{O}_{q}$ and $\operatorname{Div} M=q$ is a point. Thus the hypotheses of Part (1) hold.

We next show that $\overline{\operatorname{Div}}(T)=\overline{\operatorname{Div}}^{\ell}(T)$. The proof will be homological, using the fact that $T$ is AuslanderGorenstein and CM by Proposition 2.4 Given a (right or left) module $M$ over a ring $A$, set $E^{i}(M)=$ $E_{A}^{i}(M)=\operatorname{Ext}_{A}^{i}(M, A)$ for $i \geq 0$. We begin with some preparatory lemmas.

Lemma 7.8. Let $B=B(E, \mathcal{M}, \tau)$ where $E$ is elliptic and $\operatorname{deg} \mathcal{M} \geq 2$. For $q \in E$, define

$$
I(q)=\bigoplus H^{0}\left(E, \mathcal{M}_{n}(-q)\right), \quad I^{\prime}(q)=\bigoplus H^{0}\left(E, \mathcal{M}_{n}\left(-\tau^{-n+1}(q)\right)\right) .
$$

(1) Let $P(q)=B / I(q)$ and $P^{\prime}(q)=B / I^{\prime}(q)$. Then $\pi\left(E_{B}^{1}(P(q))\right) \cong \pi\left(P^{\prime}\left(\tau^{-2}(q)\right)[-1]\right)$ in $B$-qgr.

(2) If $P \in \operatorname{gr}-B$ with $\operatorname{GKdim}(P) \leq 1$ then $[\mathbf{c}(P)]=\left[\mathbf{c}\left(E_{B}^{1}(P)\right)\right]$ in $\overline{\operatorname{Pic}}(E)$.

Proof. (1) Let $K=E^{0}(I(q))=\operatorname{Hom}_{B}(I(q), B)$; thus $E^{1}(P(q)) \cong K / B$.

We claim that $K \doteq K^{\prime}$ where $K^{\prime}=\bigoplus_{r \geq 0} H^{0}\left(E, \mathcal{M}_{r}\left(\tau^{-r}(q)\right)\right)$. Indeed, by [AV, Theorem 1.3], $K=$ $\bigoplus_{r \geq 0} H^{0}\left(E, \mathcal{P}_{r}\right)$ for some invertible sheaves $\mathcal{P}_{r} \subset \mathbb{k}(E)$ that are ample and globally generated for $r \gg 0$. 
Now expand the equation $K_{n} I(q)_{r} \subseteq B_{n+r}$ in terms of sheaves, using the multiplication rules for $B$. This shows that $\mathcal{P}_{r}$ is defined by

$$
\mathcal{P}_{r} \otimes\left(\mathcal{O}(\mathbf{q}) \otimes \mathcal{M}_{n}\right)^{\tau^{r}} \subseteq \mathcal{M}_{n+r}
$$

Therefore, $\mathcal{P}_{r}=\mathcal{M}_{r} \otimes \mathcal{O}\left(-\tau^{-r}(\mathbf{q})\right)$. Taking sections gives the required assertion.

By Riemann-Roch, $\operatorname{dim}_{\mathbb{k}} K_{r}^{\prime}=\operatorname{dim} \mathcal{M}_{r}\left(\tau^{-r}(q)\right)=1+\operatorname{dim} B_{r}$, and $\operatorname{so} \operatorname{dim}_{\mathbb{k}}\left(K^{\prime} / B\right)_{r}=1$ for all $r \geq 1$. Also, by Lemma 2.1 $\left(K^{\prime} / B\right)_{\geq 1}$ is generated in degree one and so it is a shifted point module. Since

$$
H^{0}\left(E, \mathcal{M}_{n}\left(-\tau^{-n-1}(q)\right)\right) H^{0}\left(E, \mathcal{M}_{1}\left(\tau^{-1}(q)\right)^{\tau^{n}}\right) \subseteq H^{0}\left(E, \mathcal{M}_{n+1}\right)
$$

it follows that $I^{\prime}\left(\tau^{-2}(q)\right) \subseteq \operatorname{Ann}_{B}\left(\left(K^{\prime} / B\right)_{1}\right)$. As $B$ is generated in degree one, $\operatorname{Ann}_{B}\left(\left(K^{\prime} / B\right)_{1}\right)_{r} \neq B_{r}$, for any $r \geq 1$. Since $\operatorname{dim} B / I^{\prime}\left(\tau^{-2}(q)\right)_{m}=1$ for $m \geq 1$ it follows that $I^{\prime}\left(\tau^{-2}(q)\right)=\operatorname{Ann}_{B}\left(\left(K^{\prime} / B\right)_{1}\right)$ and $K_{\geq 1}^{\prime} \cong B / I^{\prime}\left(\tau^{-2}(q)\right)[-1]$, as required.

(2) For $Q \subseteq P$, there is an exact sequence $0 \rightarrow E^{1}(P / Q) \rightarrow E^{1}(P) \rightarrow E^{1}(Q) \rightarrow E^{2}(P / Q)$. By [Le, Theorem 6.6], $B$ is Auslander-Gorenstein and CM, whence $\operatorname{GKdim}\left(E^{2}(P / Q)\right)=2-j\left(E^{2}(P / Q)\right) \leq 0$. Thus, since $\mathbf{c}$ is computed in qgr- $B$ and is additive on short exact sequences, $\mathbf{c}\left(E^{1}(P)\right)=\mathbf{c}\left(E^{1}(Q)\right)+\mathbf{c}\left(E^{1}(P / Q)\right)$ and, analogously, for $\mathbf{c}(P)$. Thus, by induction, we can reduce to the case when $P$ is 1 -critical and hence, by Lemma 2.8, equals a shifted point module (up to finite dimensions). The result now follows from Part 1.

Lemma 7.9. Let $R=T(\mathbf{d})$ where $\operatorname{deg} \mathbf{d}<\mu$. Let $M$ be a g-torsionfree right $R$-module. Then, for $i \geq 0$, there is an isomorphism of left $\bar{R}$-modules

$$
\operatorname{Ext}_{R}^{i}(M, \bar{R}) \cong \operatorname{Ext}_{\bar{R}}^{i}(M / M g, \bar{R})
$$

Proof. By [CE Case 3, p.118], there is a homomorphism $\theta: \operatorname{Ext} \frac{i}{R}\left(M \otimes_{R} \bar{R}, \bar{R}\right) \rightarrow \operatorname{Ext}_{R}^{i}(M, \bar{R})$. Since $\theta$ is obtained by taking a projective resolution of the $R$-module $M$, it is a homomorphism of right $\bar{R}$-modules. As $M$ is $g$-torsionfree, $\operatorname{Tor}_{1}^{R}(M, \bar{R})=0$ by Lemma $7.3(2)$, while $\operatorname{Tor}_{p}^{R}(M, \bar{R})=0$ for $p>1$ since $\operatorname{pd}_{R} \bar{R}=1$. Thus, as noted in [CE, Proposition VI.4.1.3, p. 118], $\theta$ is an isomorphism.

Proposition 7.10. Let $M$ be an admissible right T-module. Then $E^{1}(M)$ is an admissible left $T$-module, and $\overline{\operatorname{Div}}(M)=\overline{\operatorname{Div}}^{\ell}\left(E^{1}(M)\right)$.

Proof. Let $M$ be an admissible right $T$-module, and let $N \subseteq M$ be the largest submodule with GKdim $N \leq 1$. Then $j(N) \geq 3-1=2$ by the $\mathrm{CM}$ condition and hence $E^{1}(N)=0$. Thus, by Lemma $7.3(5), \overline{\operatorname{Div}}\left(E^{1}(N)\right)=$ $0=\overline{\operatorname{Div}}(N)$. From the exact sequence $0 \rightarrow E^{1}(M / N) \rightarrow E^{1}(M) \rightarrow E^{1}(N)$ we obtain $E^{1}(M) \cong E^{1}(M / N)$. Thus, by Lemma $7.3(4,5), \overline{\operatorname{Div}}(M)=\overline{\operatorname{Div}}(M / N)$ and $\overline{\operatorname{Div}}\left(E^{1}(M)\right)=\overline{\operatorname{Div}}\left(E^{1}(M / N)\right)$. So, we can replace $M$ by $M / N$ and assume that $M$ is 2-pure and hence $g$-torsionfree by Lemma 7.3(1). In this case Bj], Corollary 1.22] implies that $j\left(E^{2}(M)\right) \geq 3$, whence $\operatorname{GKdim}\left(E^{2}(M)\right) \leq 0$.

Now consider the exact sequence

$$
\operatorname{Hom}_{T}(M, B) \longrightarrow E^{1}(M)[-1] \underset{27}{\stackrel{g \cdot}{\longrightarrow}} E^{1}(M) \longrightarrow \operatorname{Ext}_{T}^{1}(M, B) \longrightarrow E^{2}(M)[-1]
$$


Since $M$ is now assumed $g$-torsionfree, it follows from (7.11) and Lemma 7.9 that

$$
E^{1}(M) / g E^{1}(M) \hookrightarrow \operatorname{Ext}_{T}^{1}(M, B) \cong \operatorname{Ext}_{B}^{1}(M / M g, B)
$$

Thus final module is certainly a Goldie torsion $B$-module and so $\operatorname{GKdim}\left(\operatorname{Ext}_{B}^{1}(M / M g, B)\right) \leq 1$. In particular, $E^{1}(M)$ is also admissible.

By admissibility of $M, \operatorname{Hom}_{T}(M, B)=0$ and so (7.11) implies that $E^{1}(M)$ is $g$-torsionfree. Thus, by Lemma 7.3 $(2), \operatorname{Tor}_{T}^{1}\left(E^{1}(M), T / g T\right)=0=\operatorname{Tor}_{T}^{1}(M, T / g T)$. Hence Div $M=\mathbf{c}(M / M g)$ and $\operatorname{Div} E^{1}(M)=$ $\mathbf{c}\left(E^{1}(M) / g E^{1}(M)\right)$. Since $\operatorname{dim}_{\mathbb{K}}\left(E^{2}(M)[-1]\right)<\infty$, it follows from (7.11) that $\pi\left(E^{1}(M) / g E^{1}(M)\right) \cong$ $\pi\left(\operatorname{Ext}_{T}^{1}(M, B)\right)$. Therefore, by Lemma 7.9

$$
\operatorname{Div} E^{1}(M)=\mathbf{c}\left(E^{1}(M) / g E^{1}(M)\right)=\mathbf{c}\left(\operatorname{Ext}_{T}^{1}(M, B)\right)=\mathbf{c}\left(\operatorname{Ext}_{B}^{1}(M / M g, B)\right)=\mathbf{c}\left(E_{B}^{1}(M / M g)\right),
$$

while Div $M=\mathbf{c}(M / M g)$. Thus, to prove the result, we need only prove that $\left[\mathbf{c}_{B}(P)\right]=\left[\mathbf{c}_{B}\left(E_{B}^{1}(P)\right)\right]$ in $\overline{\operatorname{Pic}}(E)$ for any $P \in \operatorname{gr}-B$ with $\operatorname{GKdim}(P) \leq 1$. This is Lemma $\overline{7.8}(2)$.

Corollary 7.12. $\overline{\operatorname{Div}}(T)=\overline{\operatorname{Div}}^{\ell}(T)$.

Proof. By Proposition $7.10 \overline{\operatorname{Div}}(T) \subseteq \overline{\operatorname{Div}}^{\ell}(T)$ and so we are done by symmetry.

We now come to the second main result of this section, which studies the structure of $\widetilde{R} / R$ as an $R$-module for a one point blowup $R=\widetilde{R}(p)$. The result is a generalisation of [Ro, Lemma 9.1], with a different proof to allow for the fact that $R$ may not be generated in degree 1 .

Proposition 7.13. Let $R=T(\mathbf{d}+p) \subseteq \widetilde{R}=T(\mathbf{d})$ for some effective divisor $\mathbf{d}$ with $\operatorname{deg} \mathbf{d}<\mu-1$.

(1) There is a g-torsionfree line module $L$, with $\operatorname{Div}(L)=\tau(p)$, so that $\widetilde{R} / R \cong \bigoplus_{i \geq 1} L[-i]$ as right $R$-modules.

(2) The module $L$ is cyclic except in the special case where $\operatorname{deg} \mathbf{d}=\mu-2$ and $\bar{R}=B(E, \mathcal{L}, \tau)$ with $\mathcal{L} \cong \mathcal{O}_{E}(\tau(p))$, in which case $L$ is generated in degrees 0 and 1 .

(3) If $L$ is cyclic, then $L \cong R / J$ where $J=\bigoplus_{n \geq 0} M_{\widetilde{R}}(n+1, \tau(p))_{n}$.

Remark 7.14. The line module $L$ is called the right exceptional line module for the blowup $R \subseteq \widetilde{R}$. By symmetry there is also a $g$-torsionfree left line module $L^{\prime}$, so that $\widetilde{R} / R \cong \bigoplus_{i \geq 1} L^{\prime}[-i]$ as left $R$-modules. We call $L^{\prime}$ the left exceptional line module for the blowup $R \subseteq \widetilde{R}$.

Proof. $(1,2)$ In this proof all modules $J\left(\mathbf{d}^{\bullet}\right)$ and $M(k, \mathbf{d})$ are defined with respect to $\widetilde{R}$, while $\mathcal{O}_{X}=\pi(\widetilde{R}) \in$ $X=$ qgr- $\widetilde{R}$. In particular, for all $n \geq 0$ we have the right $\widetilde{R}$-ideal $M(n, p)=J\left(\mathbf{d}^{\bullet}\right)$ defined by the divisor layering $\mathbf{d}^{i}=\sum_{k=i}^{n-1} \tau^{-k}(p)$ for each $0 \leq i \leq n-1$. By definition $R=\bigoplus_{n \geq 0} M(n, p)_{n}$.

For $n \geq 0$ define a divisor layering $\mathbf{c}(j, n) \bullet$ by $\mathbf{c}(j, n)^{i}=\sum_{i \leq k \leq n-1, k \neq i+j} \tau^{-k}(p)$ for $0 \leq i \leq n-1$. (This is similar to the divisor layering for $M(n, p)$, except that the vanishings corresponding to the row $j$ (if any) in the ring $C_{p}$ are omitted.) Let $N(j, n)=J\left(\mathbf{c}(j, n)^{\bullet}\right)$ and define a right $R$-module by

$$
P^{(j)}=\bigoplus_{n \geq 0} N(j, n)_{n} \supseteq R=\bigoplus_{n \geq 0} M(n, p)_{n} .
$$


Since $\tau^{-1}\left(\mathbf{c}(j, n-1)^{i}\right)=\mathbf{c}(j, n)^{i+1}$ Lemma 4.4 (2) implies that each $P(j)$ is $g$-divisible.

We claim that $P^{(j)}$ is a right $R$-submodule of $\widetilde{R}$. Using Lemma 4.2 and Notation 3.8 write $M(n, p)=$ $\omega\left(\tau^{-n+1}\left(G_{p}\right) \circ \cdots \circ G_{p}\left(\mathcal{O}_{X}\right)\right)$. In order to show that $P_{m}^{(j)} R_{n} \subseteq P_{m+n}^{(j)}$, it suffices by Lemma 4.8(2) to show that

$$
\tau^{-m-n+1}\left(G_{p}\right) \circ \cdots \circ \tau^{-m}\left(G_{p}\right)\left[\mathcal{J}\left(\mathbf{c}(j, m)^{\bullet}\right)\right] \subseteq \mathcal{J}\left(\mathbf{c}(j, m+n)^{\bullet}\right) .
$$

Using Lemma 3.10 this is an easy exercise.

Let $M^{(j)}=P^{(j)} / R$ for each $j \geq 0$. The Hilbert series $h_{P^{(j)}}(t)$ can be calculated by Lemma 4.4 from which one sees that $M^{(j)}$ has the Hilbert series $t^{j+1} /(1-t)^{2}$ of a shifted line module. By Theorem 5.3, $R$ is $g$-divisible, whence $M^{(j)}$ is $g$-torsionfree.

We next verify that $\widetilde{R} / R \cong \bigoplus_{j \geq 0} M^{(j)}$ as right $R$-modules. First, the Hilbert series of both sides are easily seen to be the same, so it is enough to show that the submodules $M^{(i)}$ are independent. This can be done degree by degree. In degree $n$, it amounts to showing that for $0 \leq j \leq n-1$,

$$
P_{n}^{(j)} \bigcap_{\{0 \leq k \leq n-1, k \neq j\}} P_{n}^{(k)} \subseteq M(n, p)_{n}=R_{n} .
$$

By Lemma 3.6, the right $\widetilde{R}$-ideal $\sum_{\{0 \leq k \leq n-1, k \neq j\}} N(k, n)$ has image $\mathcal{J}\left(\mathbf{b}^{\bullet}\right)$ in qgr- $\widetilde{R}$, where

$$
\mathbf{b}^{\bullet}=\min _{\{0 \leq k \leq n-1, k \neq j\}} \mathbf{c}(k, n) \cdot=\left(\tau^{-j}(p), \tau^{-j-1}(p), \ldots, \tau^{-j-n+1}(p)\right) .
$$

Since $J\left(\mathbf{b}^{\bullet}\right)$ is saturated, in order to prove (7.15), it is enough to show that $N(j, n)_{n} \cap J\left(\mathbf{b}^{\bullet}\right)_{n} \subseteq R_{n}$. But $N(j, n) \cap J\left(\mathbf{b}^{\bullet}\right)=M(n, p)$ by another use of Lemma 3.6. Thus $\widetilde{R} / R \cong \bigoplus_{j \geq 0} M^{(j)}$ as claimed.

We next fix $j$ and show that $M=M^{(j)} \in$ gr- $R$. We first consider $M / M g=M / M g$. By the $g$-divisibility of $R$, we have $R \cap g P^{(j)}=g R$. It follows that $\left(R+g P^{(j)}\right) / g P^{(j)} \cong R /\left(R \cap g P^{(j)}\right)=\bar{R}$ and that

$$
\bar{M}=\frac{P^{(j)}}{R+g P^{(j)}} \cong \frac{P^{(j)} / g P^{(j)}}{\left(R+g P^{(j)}\right) / g P^{(j)}} \cong \overline{P^{(j)}} / \bar{R}
$$

where we use the $g$-divisibility of $P^{(j)}$ for the last isomorphism. Thus we need to look at the generators of

$$
\sum_{n \geq j+1}{\overline{P^{(j)}}}_{n} / \bar{R}_{n}=\bigoplus_{n \geq j+1} H^{0}\left(E, \mathcal{L}_{n}\left(\tau^{-j}(p)\right)\right) / H^{0}\left(E, \mathcal{L}_{n}\right)
$$

as a right $\bar{R}=\bigoplus_{n \geq 0} H^{0}\left(E, \mathcal{L}_{n}\right)$-module.

Consider when $\bar{M}_{n} \bar{R}_{m} \subseteq \bar{R}_{n+m}$ might hold for $n \geq j+1, m \geq 0$. Using the multiplication in $B$ in terms of sections of sheaves, this can be written as

$$
H^{0}\left(E, \mathcal{L}_{n}\left(\tau^{-j}(p)\right)\right) H^{0}\left(E, \mathcal{L}_{m}^{\tau^{n}}\right) \subseteq H^{0}\left(E, \mathcal{L}_{n+m}\right)
$$

As long as either $\operatorname{deg} \mathcal{L} \geq 2$, or $\operatorname{deg} \mathcal{L}=1$ and $m \geq 2$, Lemma 2.1 implies that $\mathcal{L}_{n+m}\left(\tau^{-j}(p)\right)$ is generated by the sections $H^{0}\left(E, \mathcal{L}_{n}\left(\tau^{-j}(p)\right)\right) H^{0}\left(E, \mathcal{L}_{m}^{\tau^{n}}\right)$, and so it cannot be contained in $H^{0}\left(E, \mathcal{L}_{n+m}\right)$. Thus (17.16) cannot hold. On the other hand, if $\operatorname{deg} \mathcal{L}=1$ and $m=1$, then $\operatorname{dim}_{\mathbb{k}} H^{0}\left(E, \mathcal{L}_{m}^{\tau^{n}}\right)=1$ and so both $H^{0}\left(E, \mathcal{L}_{n}\left(\tau^{-j}(p)\right)\right) H^{0}\left(E, \mathcal{L}_{m}^{\tau^{n}}\right)$ and $H^{0}\left(E, \mathcal{L}_{n+m}\right)$ are $n+1$-dimensional vector spaces; they will be equal if and only if $\mathcal{L}_{n}\left(\tau^{-j}(p)\right) \cong \mathcal{L}_{n+1}$. This is equivalent to $\mathcal{L} \cong \mathcal{O}_{E}\left(\tau^{n-j}(p)\right)$. In conclusion, $\bar{M}$ is cyclic and 
generated in degree $j+1$ unless $\mathcal{L} \cong \mathcal{O}_{E}(\tau(p))$, in which case it is generated in degrees $j+1$ and $j+2$. Thus, by the graded Nakayama's lemma, the same is true for $M$.

Since $M^{(j)}$ is finitely generated, it is a shifted $g$-torsionfree line module. Thus, by Lemma $7.6(2), M^{(j)}$ is critical of GK-dimension 2. It is easy to see from the calculation above that $\operatorname{Div} M^{(j)}=\tau^{-j}(p)$.

Now let $Q^{(0)}=R$ and for each $j \geq 1$ set $Q^{(j)}=P^{(0)}+P^{(1)}+\cdots+P^{(j-1)}$. Since $\widetilde{R} / R=\bigoplus M^{(j)}$, we see that $Q^{(j+1)} / Q^{(j)} \cong M^{(j)}$ as right $R$-modules. We claim that $Q^{(j)}=\sum_{n \geq 0} M\left(n-j, \tau^{-j}(p)\right)_{n}$ (recall that $M(i, \mathbf{d})=\widetilde{R}$ if $i \leq 0$.) To prove this, note that $P_{n}^{(k)}=N(k, n)_{n} \subseteq M\left(n-j, \tau^{-j}(p)\right)_{n}$ for all $0 \leq k \leq j-1$ and each $n \geq 0$, simply because the left hand side is defined by a larger divisor layering. This implies that $Q^{(j)} \subseteq \sum_{n \geq 0} M\left(n-j, \tau^{-j}(p)\right)_{n}$. But both of these modules have the same Hilbert series: the Hilbert series of $Q^{(j)}$ is clear because $Q^{(j)} / R \cong M^{(0)} \oplus \cdots \oplus M^{(j-1)}$, and the Hilbert series of $\sum_{n \geq 0} M\left(n-j, \tau^{-j}(p)\right)_{n}$ follows from Proposition 4.6. This proves the claim.

Let $L=M^{(0)}[1]$. We still must show that $M^{(i)} \cong L[-i-1]$ for $i \geq 2$. To do this, we first show that $\widetilde{R}_{1} Q^{(j)} \subseteq Q^{(j+1)}$. In degree $n+1$, we must therefore show that

$$
M\left(0, \tau^{-j-1}(p)\right)_{1} M\left(n-j, \tau^{-j}(p)\right)_{n}=\widetilde{R}_{1} Q_{n}^{(j)} \subseteq Q_{n+1}^{(j+1)}=M\left(n-j, \tau^{-j-1}(p)\right)_{n+1} .
$$

Clearly, (7.17) is a special case of Lemma 4.9(1).

It is clear that $\widetilde{R}_{j} \subseteq Q_{j}^{(j)}=M\left(0, \tau^{-j}(p)\right)_{j}$, while $\widetilde{R}_{j+1} \nsubseteq Q_{j+1}^{(j)}=M\left(1, \tau^{-j}(p)\right)_{j+1}$. If it happened that $\widetilde{R}_{1} Q^{(j)} \subseteq Q^{(j)}$, then since $\widetilde{R}$ is generated in degree 1 (see Theorem $\left[5.3\right.$ ), we would have $\widetilde{R}_{1} \widetilde{R}_{j}=\widetilde{R}_{j+1} \subseteq Q^{(j)}$, a contradiction. Thus $\widetilde{R}_{1} Q^{(j)} \nsubseteq Q^{(j)}$. We can now choose $z \in \widetilde{R}_{1}$ such that $z Q^{(j)} \nsubseteq Q^{(j)}$, but $z Q^{(j)} \subseteq Q^{(j+1)}$. Then left multiplication by $z$ induces a right $R$-module map

$$
\theta: M^{(j-1)}[-1] \cong\left(Q^{(j)} / Q^{(j-1)}\right)[-1] \rightarrow Q^{(j+1)} / Q^{(j)} \cong M^{(j)},
$$

where $\theta \neq 0$ by the choice of $z$. But each $M^{(j)}$ is 2-critical. Thus $\operatorname{ker} \theta=0$ since otherwise $\operatorname{Im}(\theta) \subset M^{(j)}$ is a nonzero submodule of GK dimension 1. Since both sides have the same Hilbert series, $\theta$ is therefore an isomorphism and $M^{(j)}[-1] \cong M^{(j+1)}$ for each $j$. In particular, each $M^{(j)} \cong L[-j-1]$ with $\operatorname{Div} L=$ $\tau\left(\operatorname{Div} M^{(0)}\right)=\tau(p)$.

(3) Let $J=\bigoplus_{n \geq 0} M(n+1, \tau(p))_{n}$. By Lemma 4.9(2), $J$ is a right ideal of $R$ while Lemma 4.4(3) implies that $\operatorname{dim}_{\mathbb{k}}(R / J)_{n} \leq n+1$ for each $n$. Now if $L[-1]=P^{(0)} / R$ as above, then Lemma 4.9 (1) implies that

$$
P_{1}^{(0)} J_{n}=M(0, p)_{1} M(n+1, \tau(p))_{n} \subseteq M(n+1, p)_{n+1}=R_{n+1} .
$$

Since $L$ is cyclic, $R / J$ surjects onto $L$. Thus they both have Hilbert series $1 /(1-t)^{2}$, and $L \cong R / J$.

\section{Minimal Sporadic ideals}

Throughout this section $T$ will be a ring satisfying Hypothesis 2.2 The aim of this section is two-fold. In both cases we will work with a slightly more restrictive class of algebras $T$, but it will include the algebras arising as Veronese subalgebras of Sklyanin algebras. The first result will be to show that $\operatorname{Div} T$ is countable. 
This will then be used to prove that $T$ has a relatively rigid ideal structure. This in turn will crucial to the proof of Theorem 1.6 in RSS.

To make this more formal, assume that $S$ is a Sklyanin-like algebra, as defined in Section 7 A graded ideal $I$ of $S$ is called sporadic if GKdim $S / I=1$. A sporadic ideal $I$ of $S$ is a minimal sporadic ideal of $S$ if every other sporadic ideal $H$ satisfies $H \supseteq I_{\geq m}$ for some $m$. When $S$ has no ideal $I$ with GKdim $S / I=1$, we declare that $S$ itself is the minimal sporadic ideal. As we will show in this section, this property does hold for our main examples.

Many of the known examples of a ring $T$ satisfying Hypothesis 2.2 arise as Veronese rings of Artin-Schelter regular algebras $S$. As we next show, $\operatorname{Div}(T)$ is countable in these cases. This result is closely related to results in $\mathrm{Aj}$ where the divisor of a module was originally defined.

Lemma 8.1. Suppose that $S$ is a Sklyanin-like algebra of finite global dimension and let $T=S^{(\gamma)}$ be the Veronese ring that satisfies Hypothesis 2.2. Then $\operatorname{Div}(T)$ is countable.

Proof. Given an admissible $S$-module $N$, the first two paragraphs of the proof of [Ro, Lemma 8.8] show that the Veronese $N^{(\gamma)}$ is an admissible $T$-module, with $\operatorname{Div}_{S} N=\operatorname{Div}_{T} N^{(\gamma)}$. Conversely, given an admissible $T$-module $M$, then $N=M \otimes_{T} S$ is an admissible $S$-module with $N^{(\gamma)} \cong M$. Thus, it suffices to prove that Div $S$ is countable. Recall from Definition $[7.2$ that $\overline{\operatorname{Pic}}(E)=\operatorname{Pic}(E) / \mathbb{Z} \alpha$, where $\tau$ is translation by $\alpha \in E$. Thus Pic $E \rightarrow \overline{\mathrm{Pic}} E$ is a countable-to-one function and so it is enough to prove that $\overline{\mathrm{Div}} S$ is countable.

By Lemma 7.3 (4,5), Div $S$ is determined by the divisors of admissible $g$-torsionfree modules $M \in$ gr- $S$. For such an $M$, pick a finite graded free resolution $F_{\bullet} \rightarrow M$. By Lemma 7.3(2) and induction, $F_{\bullet} \otimes_{S} S / g S$ is exact and hence is a graded free resolution of $M / g M$ as a module over $\bar{S}=B=B(E, \mathcal{L}, \sigma)$. Passing to qgr- $B \simeq \operatorname{coh} E$ we get a locally free resolution $\mathcal{F}_{\bullet} \rightarrow \mathcal{M}$, where $\mathcal{M}=\pi(M / M g)$ is a torsion sheaf whose support, counted with multiplicity, gives Div $M$.

The image $[\mathcal{M}]$ of $\mathcal{M}$ in the Grothendieck group $K(E)$ only depends upon the graded Betti numbers of $F_{\bullet}$. Since there are only countably many choices of these Betti numbers, there are only countably many choices for the $[\mathcal{M}]$. However, $[\mathcal{M}]=\left(\mathcal{O}_{E}(\operatorname{Div}(M)), 0\right)$, under the decomposition $K(E) \cong \operatorname{Pic} E \bigoplus \mathbb{Z}$ from [Ha, Exercise II.6.11] and so there are only countably many choices for $\operatorname{Div}(M)$.

We now turn to an arbitrary ring $T$ satisfying Hypothesis 2.2 .

Proposition 8.2. Let $T$ satisfy Hypothesis [2.2, and let $R=T(\mathbf{d}+p) \subseteq \widetilde{R}=T(\mathbf{d})$ where $\operatorname{deg} \mathbf{d}<\mu-1$. Then $\overline{\operatorname{Div}}(R)=\overline{\operatorname{Div}}(\widetilde{R})+\mathbb{Z} p$ as subsets of $\overline{\operatorname{Pic}}(E)$. In particular, if $\operatorname{Div}(\widetilde{R})$ is countable, then so is $\operatorname{Div}(R)$.

Proof. This is similar to [R0, Proposition 9.5]. As noted in the last proof, $\operatorname{Pic}(E) \rightarrow \overline{\operatorname{Pic}}(E)$ is a countableto-one function and so the second statement of the proposition follows from the first.

Given an admissible $R$-module $M$, let $\widetilde{M}=M \otimes_{R} \widetilde{R}$ and consider the exact sequence

$$
0 \rightarrow \operatorname{Tor}_{1}^{R}(M, \widetilde{R} / R) \rightarrow \underset{31}{\stackrel{\phi}{\rightarrow}} \widetilde{M} \rightarrow M \otimes_{R}(\widetilde{R} / R) \rightarrow 0
$$


Clearly $\widetilde{M} \in \operatorname{gr}-\widetilde{R}$ and it is easy to see that $\widetilde{M}$ is an admissible $\widetilde{R}$-module (see the proof of [Ro, Proposition 9.5](3)). Thus $\widetilde{M}$ is also admissible over $R$ and, by Lemma 7.4 $\operatorname{Div}_{R} \widetilde{M}=\operatorname{Div}_{\widetilde{R}} \widetilde{M}$. The outer terms of (8.3) are isomorphic as right $R$-modules to subfactors of a finite direct sum $(\widetilde{R} / R)^{(r)}$, and thus the kernel and cokernel of $\phi$ are also isomorphic to admissible $R$-module subfactors of some $(\widetilde{R} / R)^{(s)}$. Using Lemma 7.3(4), it now suffices to prove that such a module $N$ satisfies $\overline{\operatorname{Div}}(N) \in \mathbb{Z} p$. By Proposition 7.13 $\widetilde{R} / R \cong \bigoplus_{i \geq 0} L[-i-1]$ for a $g$-torsionfree line module $L$. Thus $N$ has a finite filtration by subfactors of shifts of $L$, and it suffices to prove that $\overline{\operatorname{Div}}(N) \in \mathbb{Z} p$ for a subfactor $N$ of a shift of $L$. By Lemma 7.3 (3) $\overline{\operatorname{Div}}(N[i])=\overline{\operatorname{Div}}(N)$, so we may even assume that $N$ is a subfactor of $L$. By Lemma $7.6(2)$ and Proposition 7.13. $L$ is 2-critical with Div $L=\tau(p)$. Thus, by Lemma 7.3 (4,5), either $\overline{\operatorname{Div}} N=0$ or $\overline{\operatorname{Div}} N=\overline{\operatorname{Div}} L=[p]$ in $\overline{\operatorname{Pic}}(E)$, and we are done.

We now apply the countability of Div $T(\mathbf{d})$ to study line modules and sporadic ideals. Since we may need to extend the base field, we will need that $\operatorname{Div}\left(R \otimes_{\mathbb{k}} K\right)$ is countable for some uncountable, algebraically closed field $K \supseteq \mathbb{k}$; in which case we call $\operatorname{Div}(R)$ strongly countable. Of course, this is automatic if either $\mathbb{k}$ is uncountable or, by the last two results, if $R$ is constructed from a Sklyanin-like algebra.

Lemma 8.4. Let $R=T(\mathbf{d})$ for some effective $\mathbf{d}$ with $\operatorname{deg} \mathbf{d}<\mu$. Assume that $\operatorname{Div}(R)$ is strongly countable. Then the set $\left\{\operatorname{Div} L \mid L_{R}\right.$ is a g-torsionfree cyclic line module $\}$ is finite.

Proof. This is very similar to the proof of [R0, Theorem 9.7], the main difference being that line modules are automatically $g$-torsionfree and cyclic in the context of that paper. In contrast, Example 8.9 shows that line modules can even be $g$-torsion in our case.

The ring $R$ is strongly noetherian by Theorem 5.3 and so, by [AZ2, isomorphism classes of cyclic line modules over $R$ are parametrised by a projective scheme, the line scheme. More specifically, for each $m$ the set of graded subspaces $\bigoplus_{j=0}^{m} J_{j} \subseteq \bigoplus_{j=0}^{m} R_{j}$ such that $\operatorname{dim} J_{i}=\operatorname{dim} R_{i}-i$ for each $i$, and such that $J_{i} R_{j} \subseteq J_{i+j}$ for all $i, j$ with $i+j \leq m$, is parametrised by a projective scheme $X_{m}$. There is a truncation map $\phi_{m}: X_{m+1} \rightarrow X_{m}$ for each $m$ induced by forgetting the $(m+1)^{\text {st }}$ coordinate, and [AZ2, Corollary E4.5] implies that there is $m_{0} \in \mathbb{N}$ so that $\phi_{m}$ is an isomorphism for $m \geq m_{0}$. Then $X_{m_{0}}$ is the line scheme.

If $L=R / J$ is a line module, then $L / L g$ has Hilbert series at least as large as the Hilbert series $(1-t)^{-1}$ of a point module; and it has the Hilbert series of a point module if and only if $L$ is $g$-torsionfree. As we run over the set of $J$ 's such that $R / J$ is a line module, $\operatorname{dim}_{\mathbb{k}} J_{m}+g R_{m-1}$ is a lower semi-continuous function with maximum value $\operatorname{dim}_{\mathbb{k}} R_{m}-1$. Thus requiring $\operatorname{dim}_{\mathbb{k}} J_{m}+g R_{m-1}=\operatorname{dim}_{\mathbb{k}} R_{m}-1$ for any fixed $m$ is a further open condition on the set of such $J$. Now apply [AZ2, Corollary E4.5(2)] to the Hilbert series of a point module. It shows that there is $n$ such that for any right ideal $I$ of $R$ such that $\operatorname{dim}_{\mathbb{k}}(R / I)_{m}=1$ for all $0 \leq m \leq n$, then $\operatorname{dim}_{\mathbb{k}}(R / I)_{m}=1$ for all $m \geq 0$ (and so $R / I$ is a point module). This implies that the condition that a line module $R / J$ be $g$-torsionfree can be tested in a fixed finite set of degrees. Thus it is an intersection of finitely many open conditions, and so the set of $g$-torsionfree line modules is an open subset $W$ of the line scheme; in particular it is a $\mathbb{k}$-scheme of finite type. 
The rest of the proof is basically the same as [Ro, Theorem 9.7], simply using $W$ in place of the entire line scheme, but we sketch some of the details for the reader's convenience. As in that proof, we can extend the base field if necessary and assume that $\mathbb{k}$ is uncountable. There is a map $\theta: W \rightarrow E$ sending a $g$-torsionfree cyclic line module $L=R / J$ to its divisor Div $L$, which is the single point $p \in E$ corresponding to the point module $P(p)=L / g L$. For any fixed $q \in E$, Div $L=q$ if and only if $J+g R=I$, where $P(q)=R / I$. Thus the fibre $\theta^{-1}(q)$ is a closed subset in $W$. Since Div $T$ is countable, $W$ is therefore a union of countably many proper closed subsets. By, for example, [NS, Lemma 6.5] this forces the union to be finite. Equivalently, the set of divisors associated to $g$-torsionfree cyclic line modules must be finite.

Remark 8.5. Note that Div $T$ can be uncountable for algebras satisfying Hypothesis 2.2. For example, consider $T=B(E, \mathcal{M}, \tau)[g]$, where $g$ is an indeterminate in degree one and, as usual, $\operatorname{deg} \mathcal{M} \geq 2$. For each point $p \in E$ and corresponding right point module $P(p)=B / I$, the $T$-module $L=T / I[g]$ is a $g$-torsionfree line module with $\operatorname{Div} L=p$. Hence $\{\operatorname{Div} L \mid L$ is a $g$-torsionfree line module $\}$ has cardinality at least as large as that of $E$. Thus for this example, Lemma 8.1 fails if $\mathbb{k}$ is uncountable. On the other hand, if $\mathbb{k}$ is countable then it shows that Lemma 8.4 can fail if one replaces the strongly countable hypothesis by a countable one.

We now turn to the study of sporadic ideals. The punchline of the proof, Proposition 8.7 shows how the minimal sporadic ideal for $R=T(\mathbf{d}+p)$ is determined by the minimal sporadic ideal for $\widetilde{R}=T(\mathbf{d})$, together with the structure of the $g$-torsionfree GK-1 factor modules of the right and left exceptional line modules coming from Proposition 7.13 The next result shows how to control these factors. The first part of the next result is a generalisation of [Ro, Lemma 10.1].

Lemma 8.6. Let $R=T(\mathbf{d})$ where $T$ satisfies Hypothesis 2.2 and $\operatorname{deg} \mathbf{d}<\mu$, and assume that the set $\{$ Div $M \mid M$ is a g-torsionfree cyclic line module for $R$ \} is finite. Let $L$ be any $g$-torsionfree (not necessarily cyclic) line module for $R$.

(1) $L$ has a unique smallest submodule $N$ such that $L / N$ is g-torsionfree with $\operatorname{GKdim} L / N=1$.

(2) Consider the right annihilator $I=\mathrm{r}-\operatorname{ann}(L / N)$. Then $I$ is a sporadic ideal that annihilates every finitely generated $g$-torsionfree subfactor $M$ of a direct sum of shifts of $L$ with GKdim $M=1$.

Proof. (1) A module $N \subset L$ such that $L / N$ is $g$-torsionfree of GK-dimension 1 will be called a good submodule. To prove the result, it suffices to prove that $L$ has DCC on good submodules. For any good submodule $N$, $\operatorname{dim}_{\mathbb{k}} L /(N+L g)<\infty$ and so $\operatorname{dim}_{\mathbb{k}}(L / N)_{n}$ is a constant, say $d$, for $n \gg 0$. We call $d$ the multiplicity of $L / N$. To prove Part (1) it also suffices to show that the possible multiplicity of $L / N$ for good submodules $N$ is bounded above.

Set $B=R / g R$ and consider $P=L / L g \in \operatorname{gr}-B$. Then $h_{P}(t)=1 /(1-t)$; so $P$ is a (not necessarily cyclic) point module. We claim that there is $n_{0} \geq 0$ such that every nonzero submodule of $P_{\geq n_{0}}$ is a shifted cyclic point module. Write $R / R g=B(E, \mathcal{L}, \tau)$, where $\mathcal{L}=\mathcal{M}(-\mathbf{d})$. By Lemma 2.8 $(1,2)$, there is a point module of the form $P(p)=\bigoplus_{n \geq 0} H^{0}\left(E, \mathbb{k}(p) \otimes \mathcal{L}_{n}\right)$ such that $P_{\geq m} \cong P(p)_{\geq m}$. Thus we can replace $P$ by 
$P(p)$. Now if $P_{m} B_{1}=0$, for some $m \geq 0$, then by the definition of multiplication in $B$, the image of the multiplication map $f: H^{0}\left(E, \mathcal{L}_{m}\right) \otimes H^{0}\left(E, \mathcal{L}^{\tau^{m}}\right) \rightarrow H^{0}\left(E, \mathcal{L}_{m+1}\right)$ is contained in $H^{0}\left(E, \mathcal{I}_{p} \otimes \mathcal{L}_{m+1}\right)$, where $\mathbb{k}(p)=\mathcal{O}_{E} / \mathcal{I}_{p}$. If $\operatorname{deg} \mathcal{L} \geq 2$ this never happens, by Lemma 2.1. If $\operatorname{deg} \mathcal{L}=1$, then by a similar argument as in Proposition 7.13, this happens if and only if $\mathcal{L}^{\tau^{m}} \cong \mathcal{O}_{E}(p)$. This can happen for at most one $m \geq 2$. Thus some tail $P_{\geq n_{0}}$ has the property that $P_{i} B_{1}=P_{i+1}$ for all $i \geq n_{0}$. In particular, every nonzero submodule of $P_{\geq n_{0}}$ is a shifted cyclic point module, proving the claim.

Suppose that $N \subset L$ is a good submodule with $N_{\leq n_{0}}=0$, where $n_{0}$ is defined by the last paragraph. Tensoring $0 \rightarrow N \rightarrow L \rightarrow L / N \rightarrow 0$ with $R / R g$ gives an exact sequence $0 \rightarrow N / N g \rightarrow L / L g \rightarrow L /(N+$ $L g) \rightarrow 0$, by Lemma 7.3(2). By definition, $N / N g \subseteq(L / L g)_{\geq n_{0}}$, and so is a shifted cyclic point module. Since $N$ is $g$-torsionfree, this implies that $N$ is a shifted cyclic line module. Now Div $L / N=0$, since $L / N$ is $g$-torsionfree with $\operatorname{GKdim}(L / N)=1$. Thus $\operatorname{Div} L=\operatorname{Div} N=p$, by Lemma 7.3(4). Writing $N \cong L^{\prime}[-i]$ for a line module $L^{\prime}$, then $\operatorname{Div} L^{\prime}=\tau^{i}(p)$ by Lemma 7.3(3). We have therefore found a $g$-torsionfree cyclic line module for $R$ with divisor $\tau^{i}(p)$. Note that $i$ is also the multiplicity of $L / N$. Since by assumption the set of divisors associated to $g$-torsionfree cyclic line modules is finite, there is an upper bound on $i$ and thus on the multiplicity of $L / N$ for good submodules $N$ with $N_{\leq n_{0}}=0$.

Now suppose that we have a descending chain $N^{(1)} \supseteq N^{(2)} \supseteq \ldots$ of good submodules of $L$. If $N_{\leq n_{0}}^{(i)}=0$ for some $i$, then for all $j>i$ the multiplicity of $L / N^{(j)}$ is bounded above by the previous paragraph, and thus the chain must stabilise. Otherwise $N_{\leq n_{0}}^{(i)} \neq 0$ for all $i$; but this implies that the exists a fixed finitedimensional nonzero vector subspace $X$ of $L$ such that $N_{\leq n_{0}}^{(i)}=X$ for all $i \gg 0$. The submodule $X R$ of $L$ is contained in every $N^{(i)}$, and GKdim $L / X R=1$ since $L$ is 2-critical by Lemma 7.6(2). Thus the multiplicity of $L / X R$ is an upper bound for the multiplicity of all the $L / N^{(i)}$, and again the chain stabilises.

(2) Exactly the same proof as in [Ro, Lemma 10.2] applies.

Proposition 8.7. Let $T$ satisfy Hypothesis 2.2 and suppose in addition that $\operatorname{Div}(T)$ is strongly countable, and that $T$ has a minimal sporadic ideal. Let $R=T(\mathbf{c})$ where $\operatorname{deg} \mathbf{c}<\mu$.

(1) $\operatorname{Div}(R)$ and $\operatorname{Div}^{\ell}(R)$ are also strongly countable.

(2) $R$ has a minimal sporadic ideal, which can be determined inductively as follows. Write $R=T(\mathbf{d}+p) \subseteq$ $\widetilde{R}=T(\mathbf{d})$, where $\operatorname{deg} \mathbf{d} \leq \mu-2$. Suppose that $H$ is a minimal sporadic ideal of $\widetilde{R}$. Let $L$, respectively $L^{\prime}$, be the right, respectively left, exceptional line module for the blowup $R \subseteq \widetilde{R}$, as in Remark 7.14 . Then Lemma 8.6(2) holds for $L$ on the right and $L^{\prime}$ on the left, and produces sporadic ideals $I$, respectively $G$. Then $G(H \cap R) I$ is a minimal sporadic ideal for $R$.

Proof. (1) Take an uncountable algebraically closed field extension $K \supseteq \mathbb{k}$. By Remark [5.9, $T(\mathbf{c}) \otimes_{\mathbb{k}} K=$ $\left(T \otimes_{\mathbb{k}} K\right)(\mathbf{c})$ for any divisor $\mathbf{c}$ and so the earlier results apply to $R \otimes_{\mathbb{k}} K$. In particular, by Proposition 5.4 we can form $R \otimes_{\mathbb{k}} K$ iteratively via a series of one point blowups starting with $T \otimes_{\mathbb{k}} K$. Then $\operatorname{Div}(R)$ is strongly countable by Proposition 8.2 and induction. By Corollary 7.12 and induction on a left-sided version of Proposition 8.2, $\operatorname{Div}^{\ell}(R)$ is strongly countable. 
(2) Clearly it suffices to work inductively as stated. By Lemma 8.4 the hypotheses of Lemma 8.6 hold for $R$ and $L$, and thus Lemma 8.6 (2) produces a sporadic ideal $I$ which kills all $g$-torsionfree subfactors $N$ of direct sums of shifts of $L$ with $\operatorname{GKdim} N=1$. The same argument on the left constructs $G$. Now the proof of [Ro, Theorem 10.4] applies, and shows that $G(H \cap R) I$ is a minimal sporadic ideal for $R$.

The following theorem shows that the previous result applies to the most important examples of interest.

Theorem 8.8. Let $T=S^{(\gamma)}$, where $S$ is a generic cubic or quadratic Sklyanin algebra, with central element $g \in S_{\gamma}$. Then $T$ satisfies the hypothesis of Proposition 8.7 (in fact, $T$ has no sporadic ideals). Thus every blowup $R=T(\mathbf{d})$ with $\operatorname{deg} \mathbf{d}<\mu$ has a minimal sporadic ideal.

Proof. Let $K \supseteq \mathbb{k}$ be an uncountable algebraically closed field extension. It is clear that $S \otimes_{\mathbb{k}} K$ is still a Sklyanin algebra, since the defining relations do not depend upon the choice of field and it is still generic by [ATV, Theorem 7.1]. Hence it is a Sklyanin-like algebra of global dimension 3. Thus $\operatorname{Div}(T)$ is strongly countable by Lemma 8.1 Further, [ATV, Theorem I] shows that $S\left[g^{-1}\right]_{0}=T\left[g^{-1}\right]_{0}$ is simple. Since $T / I$ cannot be $g$-torsion for a special ideal $I$, it follows that $T$ cannot have a sporadic ideal. Thus $T$ satisfies the hypotheses of Proposition 8.7 .

To end the section, we give an example to show that the complexities in Lemma 8.4 are in fact needed: for the rings $R=T(\mathbf{d})$ where $\mathbf{d}$ has degree $\mu-1$, a line module need not be $g$-torsionfree nor 2-critical in general. If $\operatorname{deg} \mathbf{d}<\mu-1$, then, as previously remarked, all line modules over $T(\mathbf{d})$ are $g$-torsionfree.

Example 8.9. Let $R=T(\mathbf{d})$ where $T$ satisfies Hypothesis 2.2 and $\mathbf{d}$ has degree $\mu-1$. Then there exists a cyclic line module $M \in \mathrm{gr}-R$ which is $g$-torsion, and which has a shifted cyclic point module as a submodule.

Proof. By Theorem 5.3(1), T(d) has Hilbert series $h_{T(\mathbf{d})}(t)=\frac{t^{2}-t+1}{(1-t)^{3}}$ and so $h_{B}(t)=\frac{t^{2}-t+1}{(1-t)^{2}}$ for $B=T / g T$. Now consider $M=R / g^{2} R$. As $g^{2}$ is regular of degree $2, h_{M}(t)=\frac{t^{2}-t+1}{(1-t)^{3}}\left(1-t^{2}\right)=\frac{1+t^{3}}{(1-t)^{2}}$. Pick any $0 \neq x \in M$ that lies in degree 2 in the submodule $g R / g^{2} R \cong B[-1]$. Then $x B=x R \cong B[-2]$ has Hilbert series $\frac{t^{2}\left(t^{2}-t+1\right)}{(1-t)^{2}}$ and so $x B_{+}=x R_{+}$has the Hilbert series $\frac{t^{2}\left(t^{2}-t+1\right)}{(1-t)^{2}}-t^{2}=\frac{t^{3}}{(1-t)^{2}}$ of a shifted line module.

Finally, set $L=M / x R_{+}=R /\left(g^{2} R+x R_{+}\right)$. This is cyclic with $h_{L}(T)=\frac{1+t^{3}-t^{3}}{(1-t)^{2}}=\frac{1}{(1-t)^{2}}$. If $L^{\prime}=$ $g R /\left(g^{2} R+x R_{+}\right) \subset L$, then $\bar{L}=L / L^{\prime} \cong B$. Moreover $L^{\prime}=g R /\left(g^{2} R+x R_{+}\right)$is also cyclic with $h_{L^{\prime}}(t)=\frac{t}{(1-t)}$, so $L^{\prime}$ is a shifted point module.

\section{ON THE NONEXISTENCE OF SPORADIC IDEALS}

In Theorem 8.8 we exhibited a large number of algebras with a minimal special ideal, and this leads to a natural question: when do these algebras $T$ have no sporadic ideal? The question is delicate in general, and a partial answer is given in [VB2, Section 11] when $T=S^{(3)}$ for the quadratic Sklyanin algebra $S$, but the answer uses much of the machinery of that paper. In this section, we give an elementary argument which can be used to answer the question in a few easy cases. We restrict our attention to the following situation. 
Notation 9.1. Throughout the section, we fix a ring $T$ satisfying Hypothesis 2.2. Let $\mathbf{d}$ be an effective divisor on $E$ with $\operatorname{deg} \mathbf{d}<\mu-2$, and let $p \in E$. Set $\widetilde{R}=T(\mathbf{d})$ and $R=T(\mathbf{d}+p)$; thus $R \cong \widetilde{R}(p)$ by Proposition 5.4. Also, by Theorem 5.3, $\widetilde{R}$ and $R$ are generated in degree 1. Throughout $M(k, p)=M_{\widetilde{R}}(k, p)$ in the notation of Definition 4.1 and $M^{\prime}(k, p)=M_{\widetilde{R}}^{\prime}(k, p)$ in the notation of Definition 6.2 .

Proposition 9.2. Keep $R=\widetilde{R}(p) \subseteq \widetilde{R}$ as in Notation 9.1 and assume that $\widetilde{R}$ has no sporadic ideals and that $\operatorname{Div}(\widetilde{R})$ is strongly countable. Suppose in addition that:

(C1) for $q \in\left\{\tau^{i}(p) \mid i \leq 1\right\}$, there exist $k \geq 1$ and $K \subseteq \widetilde{R}_{k}$ with $K \widetilde{R}_{1}=\widetilde{R}(q)_{k+1}=M(k+1, q)_{k+1}$;

(C2) for $q \in\left\{\tau^{-i}(p) \mid i \leq 1\right\}$, there exist $k \geq 1$ and $K \subseteq \widetilde{R}_{k}$ with $\widetilde{R}_{1} K=\widetilde{R}(q)_{k+1}=M^{\prime}(k+1, q)_{k+1}$.

Then $R$ has no sporadic ideals.

Before proving the proposition, we make some comments about its statement and give a lemma.

Remark 9.3. (1) Since $M(k+1, q)$ is a saturated right ideal of $\widetilde{R}$, if condition (C1) holds for $K$ then necessarily $K \subseteq M(k+1, q)_{k}$ and so the condition is also satisfied by $K^{\prime}=M(k+1, q)_{k}$. Thus $(\mathrm{C} 1)$ is equivalent to saying that $M(k+1, q)$ can be generated in one degree lower than is necessary (it is generated in degree $k+1$ by Lemma 4.9).

(2) If (C1) holds for some $k$ and $K$, then we can also satisfy (C1) for any larger $k$; indeed, if $U=\widetilde{R}(q)$ then $\left[U_{m} K\right] \widetilde{R}_{1}=U_{m} U_{k+1}=U_{k+1+m}$ for any $m \geq 0$.

Lemma 9.4. Let $\widetilde{R}=T(\mathbf{d})$ as above and assume that (C1) and (C2) hold.

(1) For any $\ell \geq 1$, there is $m \geq 1$ and $K \subseteq \widetilde{R}_{m}$ such that $K \widetilde{R}_{\ell}=R_{\ell+m}$.

(2) For any $\ell \geq 1$ there is $m^{\prime} \geq 1$ and $K^{\prime} \subseteq \widetilde{R}_{m^{\prime}}$ such that $\widetilde{R}_{\ell} K^{\prime}=R_{\ell+m^{\prime}}$.

Proof. By symmetry, we need only prove Part (1). When $\ell=1$, this is just (C1) with $q=p$. So, by induction, suppose the result holds for some $\ell \geq 1$, say for $K \subseteq \widetilde{R}_{m}$. Choose $k \geq 1$ such that (C1) holds for $q=\tau^{-\ell}(p)$. By Remark $9.3(1)$, this is equivalent to $M\left(k+1, \tau^{-\ell}(p)\right)_{k} \widetilde{R}_{1}=M\left(k+1, \tau^{-\ell}(p)\right)_{k+1}$. Now Corollary 4.14 implies that

$$
M\left(k+1, \tau^{-\ell}(p)\right)_{k+1} \widetilde{R}_{\ell}=M\left(k+1, \tau^{-\ell}(p)\right)_{k+\ell+1}=\widetilde{R}_{\ell} M(k+1, p)_{k+1} .
$$

Finally, we calculate

$$
\begin{aligned}
K M\left(k+1, \tau^{-\ell}(p)\right)_{k} \widetilde{R}_{\ell+1}= & K M\left(k+1, \tau^{-\ell}(p)\right)_{k} \widetilde{R}_{1} \widetilde{R}_{\ell}=K M\left(k+1, \tau^{-\ell}(p)\right)_{k+1} \widetilde{R}_{\ell} \\
& =K \widetilde{R}_{\ell} M(k+1, p)_{k+1}=R_{\ell+m} R_{k+1}=R_{\ell+m+k+1}
\end{aligned}
$$

proving the induction step.

Proof of Proposition 9.2. By Proposition 8.7 applied to the ring $\widetilde{R}$, the minimal sporadic ideal of $R$ has the form $G(H \cap R) I$ where $H$ is the minimal sporadic ideal of $\widetilde{R}$, and $G$ (respectively $I$ ) is the sporadic ideal of $R$ which kills all $g$-torsionfree proper factors of the left (respectively right) exceptional line module for the blowup $R \subseteq \widetilde{R}$. By hypothesis, $H=\widetilde{R}$ and so, by symmetry, it suffices to prove that $I=R$. 
Let $L=R / J$ be the exceptional right line module for the blowup $R \subseteq \widetilde{R}$. By Proposition $7 \mathbf{7 . 1 3}(2,3), L$ is cyclic and $J=\bigoplus_{n \geq 0} M(n+1, \tau(p))_{n}$. Let $x \in R_{n} \backslash J_{n}$ for some $n \geq 0$. If we show that $R /(J+x R)$ is $g$-torsion for all such $x$, then $L$ will have no $g$-torsionfree proper factors and we will be done. It is enough to prove this for all $n \gg 0$, since any nonzero submodule of $R / J$ will contain elements of all large degrees.

Now we use condition (C1) for $q=\tau(p)$ and some fixed $k$. By Remark 9.3(1,2), for $n \geq k$ the right $\widetilde{R}$-module $M(n+1, \tau(p))$ is generated in degrees $\leq n$. Thus $J_{n} \widetilde{R}=M(n+1, \tau(p))_{\geq n}$ if $n \geq k$.

Let $V=R_{n} \widetilde{R} / J_{n} \widetilde{R}$ for some $n \geq k$. Then $R_{n} \widetilde{R}=M(n, p)_{n} \widetilde{R}=M(n, p)_{\geq n}$, using Lemma 4.9. Hence $V \cong[M(n, p) / M(n+1, \tau(p))]_{\geq n}$ and so, by Theorem $3.1 \widehat{\pi(V)}_{p}$ is isomorphic to a left-infinite row vector

$$
(\ldots, 0,0, \ldots, \overbrace{k[[x]] /(x), \ldots, k[[x]] /(x)}^{n+1})
$$

with the obvious right $C_{p}$-action. This is a non-zero uniserial $C_{p}$-module, and so $\pi(V)$ is a uniserial object in qgr- $\widetilde{R}$. Since $x \in R_{n} \backslash J_{n}$, the $\widetilde{R}$-module $W=\left(x \widetilde{R}+J_{n}\right) / J_{N} \widetilde{R} \subseteq V$ is non-zero and hence is infinite dimensional since $M(n+1, \tau(p))$ is saturated. Thus $\pi(W) \neq 0$ and so must contain the simple socle of $\pi(V)$. This socle is easily seen to be $\pi\left[\left(g^{n} \widetilde{R}+J_{n} \widetilde{R}\right) /\left(J_{n} \widetilde{R}\right)\right]$. Hence $g^{n} \widetilde{R}_{\ell} \subseteq x \widetilde{R}_{\ell}+J_{n} \widetilde{R}_{\ell}$ for some $\ell$.

Now use Lemma 9.4(2) to write $\widetilde{R}_{\ell} K=R_{\ell+m}$, for some $K \subseteq \widetilde{R}_{m}$. Then $J_{n} \widetilde{R}_{\ell} K+x \widetilde{R}_{\ell} K \supseteq g^{n} \widetilde{R}_{\ell} K$, or in other words $J_{n} R_{\ell+m}+x R_{\ell+m} \supseteq g^{n} R_{\ell+m}$. Thus $R /(J+x R)$ is $g$-torsion, and we are done.

Now we give a few examples where the criteria of the proposition can be checked. Many of these results can also be obtained from [VB2, Section 11], but we prefer to give elementary proofs.

Example 9.5. Let $T=S^{(3)}$ for a generic quadratic Sklyanin algebra $S$. Then $T(p)$ has no sporadic ideals.

Proof. By Theorem 8.8 and its proof, $T$ has no sporadic ideals and $\operatorname{Div}(T)$ is strongly countable. Recall that $S / g S \cong B(E, \mathcal{L}, \sigma)$, and so $T / g T \cong B(E, \mathcal{M}, \tau)$ where $\tau=\sigma^{3}$ and $\mathcal{M}=\mathcal{L} \otimes \mathcal{L}^{\sigma} \otimes \mathcal{L}^{\sigma^{2}}$. For $q \in E$, the subspace $W(q)=H^{0}(E, \mathcal{L}(-q)) \subseteq H^{0}(E, \mathcal{L})=S_{1}$ is called a point space. By [Ro, Lemma 4.2], $W(q) S=I$, where $S / I$ is the point module corresponding to $q$. In particular, $W(q) S_{2}=M(2, q)_{1}=T(q)_{1}$. (Note that a subscript on a graded piece of $S$ indicates the $S$-degree, but a subscript on a right ideal of $T$ indicates the $T$-degree.) We also have $W(q) S_{1}=S_{1} W(\sigma(q))$ by [Ro, Lemma 4.1(1)], or by Lemma 2.1 Then for any $q$,

$$
W(q) W\left(\sigma^{-2}(q)\right) S_{1} S_{3}=\left[W(q) S_{2}\right]\left[W(q) S_{2}\right]=T(q)_{2}=M(2, q)_{2} .
$$

This shows that (C1), and by symmetry (C2), holds for all $q \in E$. Now apply Proposition 9.2

Example 9.6. Let $T=S^{(3)}$ as in Example 9.5. Suppose that $p$ and $r$ are points on distinct $\tau$-orbits. Then $T(p+r)$ has no sporadic ideals.

Proof. By [Ro, Lemma 4.1(2)] or Lemma 2.1, the point spaces satisfy $W(p) W(\sigma(q))=W(q) W(\sigma(p))$ whenever $p \neq \tau(q)$ and $q \neq \tau(p)$. 
Write $\widetilde{R}=T(r)$ and $R=\widetilde{R}(p)=T(p+r)$. Note that $\widetilde{R}_{1}=W(r) S_{2}$ as in the previous example and that $\widetilde{R}(q)_{1}=W(q) W(\sigma(r)) S_{1}$ by [Ro, Lemma 4.6(1)]. Then for any $q$ on the $\tau$-orbit of $p$,

$$
\begin{gathered}
{\left[W(q) W\left(\sigma^{-2}(q)\right) W\left(\sigma^{2}(r)\right)\right] \widetilde{R}_{1}=\left[W(q) W(\sigma(r)) W\left(\sigma^{-1}(q)\right)\right]\left[W(r) S_{2}\right]} \\
\quad=\left[W(q) W(\sigma(r)) S_{1}\right]\left[W(q) W(\sigma(r)) S_{1}\right]=\widetilde{R}(q)_{1} \widetilde{R}(q)_{1}=\widetilde{R}(q)_{2} .
\end{gathered}
$$

Thus (C1) holds, and the proof of $(\mathrm{C} 2)$ is analogous. By Example $9.5 \widetilde{R}$ has no sporadic ideals, and $\operatorname{Div}(\widetilde{R})$ is strongly countable by Proposition 8.2 so the result follows from Proposition 9.2 .

It is not hard to show on the other hand that $T\left(p+\sigma^{i}(p)\right)$ always has a sporadic ideal if $i \neq 0$ (see Ro, Proposition 11.2(1)]). We expect that $T(2 p)$ has no sporadic ideals, though Proposition 9.2 does not apply.

Example 9.7. Suppose instead that $T=S^{(4)}$ for the generic cubic Sklyanin algebra $S$, with central element $g \in S_{4}$. Then $T(p)$ has no sporadic ideals for any point $p \in E$.

Proof. The proof is similar to the previous examples. Again, $T$ has no sporadic ideals, and $\operatorname{Div}(T)$ is strongly countable, by Theorem 8.8. For $q \in E$, set $V(q)=H^{0}\left(E, \mathcal{L}_{2}(-q)\right) \subseteq H^{0}\left(E, \mathcal{L}_{2}\right)=S_{2}$. The rule $V(q) S_{1}=S_{1} V(\sigma(q))$ follows from Lemma 2.1 while, $T(q)_{1}=V(q) S_{2}$ follows from [ATV, Proposition 6.7]. Then

$$
\left[V(q) V\left(\sigma^{-2}(q)\right)\right] T_{1}=V(q) S_{2} V(q) S_{2}=T(q)_{2} .
$$

Since this holds for all $q$, condition (C1) is verified and (C2) is similar. Now apply Proposition 9.2 .

\section{INDEX OF NOTATION}

$\doteq$, equal in high degree

$\alpha$-pure

Admissible module

Allowable divisor layering $\mathbf{d}^{\bullet}$

Auslander Gorenstein and CM

$\mathbf{c}(M)$ divisor

Div, $\overline{\mathrm{Div}}, \overline{\mathrm{Pic}}$

$\operatorname{Div}^{\ell}, \overline{\operatorname{Div}}^{\ell}$

$\mathbf{d}_{n}=\mathbf{d}+\mathbf{d}^{\tau}+\cdots+\mathbf{d}^{\tau^{n-1}}$ for a divisor $\mathbf{d}$

$E$ elliptic curve

Exceptional line modules

Functors $F_{q}, G_{\mathbf{d}}$

$g$-divisible

Line module

$\mathfrak{J}\left(\mathbf{d}^{\bullet}\right), \mathcal{J}\left(\mathbf{d}^{\bullet}\right), J\left(\mathbf{d}^{\bullet}\right)$
$M(k, \mathbf{d})$

$\mu=\operatorname{deg} \mathcal{M}$

Point module, shifted point module

$\pi: \operatorname{gr}-R \rightarrow$ qgr- $R$

$Q(i, r, d, p)$

Section functor $\omega$,

Saturation $I^{\text {sat }}$, saturated right ideal

Sklyanin algebras

Sklyanin-like algebra

Sporadic ideal, minimal sporadic ideal

Strongly countable

$T, T / g T \cong B(E, \mathcal{M}, \tau)$

$T(\mathbf{d}), T_{\ell} * T(\mathbf{d})$
12

5

6

6

22

6

6 


\section{REFERENCES}

[Aj] K. Ajitabh, Modules over elliptic algebras and quantum planes, Proc. London Math. Soc. 72 (1996), 567-587.

[Ar] M. Artin, Some problems on three-dimensional graded domains, in: Representation Theory and Algebraic Geometry (Waltham, MA, 1995), 1-19, London Math. Soc. Lecture Note Ser., no. 238, Cambridge Univ. Press, Cambridge, 1997.

[ATV] M. Artin, J. Tate, and M. Van den Bergh, Modules over regular algebras of dimension 3, Invent. Math. 106 (1991), 335-388.

[AV] M. Artin and M. Van den Bergh, Twisted homogeneous coordinate rings J. Algebra 133 (1990), 249-271.

[AZ1] M. Artin and J. J. Zhang, Noncommutative projective schemes, Adv. Math. 109 (1994), no. 2, 228-287.

[AZ2] M. Artin and J. J. Zhang, Abstract Hilbert schemes, Algebr. Represent. Theory 4 (2001), 305-394.

[Bj] J. E. Bjork, Filtered noetherian rings, in Noetherian rings and their applications, Ed. L. W. Small, Math. Surveys and Monographs, Amer. Math. Soc. No. 24, Providence, RI, 1987.

[CE] H. Cartan and S. Eilenberg, Homological algebra, Princeton University Press, Princeton, NJ, 1956.

[Ha] R. Hartshorne, Algebraic geometry, Springer-Verlag, Berlin, 2006.

[Le] T. Levasseur, Some properties of noncommutative regular graded rings, Glasgow Math. J. 34 (1992), 277-300.

[MR] J. C. McConnell and J. C. Robson, Noncommutative Noetherian Rings, Graduate Studies in Math., Vol. 30, Amer. Math. Soc., 1987.

[NS] T. A. Nevins and S. J. Sierra, Naïve blowups and canonical birationally commutative factors, Math. Z. 280 (2015), $1125-1161$.

[NV] C. Năstăsescu and F. Van Oystaeyen, Graded Ring Theory, North Holland, Amsterdam, 1982.

[Ro] D. Rogalski, Blowup subalgebras of the Sklyanin algebra, Advances in Math. 226 (2011), 1433-1473

[RSS] D. Rogalski, S. J. Sierra and J. T. Stafford, Classifying orders in the Sklyanin algebra, arXiv:1308.2213 To appear in Algebra and Number Theory.

[St] D. R. Stephenson, Algebras associated to elliptic curves, Trans. Amer. Math. Soc. 349 (1997), 2317-2340.

[VB1] M. Van den Bergh, Existence theorems for dualizing complexes over non-commutative graded and filtered rings, J. Algebra 195 (1997), 662-679.

[VB2] M. Van den Bergh, Blowing up of non-commutative smooth surfaces, Mem. Amer. Math. Soc., 154 (2001), no. 734.

[VV] M. Van Gastel and M. Van den Bergh, Graded modules of Gelfand-Kirillov dimension one over three-dimensional Artin-Schelter regular algebras, J. Algebra 196 (1997), 251-282.

(Rogalski) Department of Mathematics, UCSD, La Jolla, CA 92093-0112, USA.

E-mail address: drogalsk@math.ucsd.edu

(Sierra) School of Mathematics, University of Edinburgh, Edinburgh EH9 3JZ, U.K.

E-mail address: s.sierra@ed.ac.uk

(Stafford) School of Mathematics, The University of Manchester, Manchester M13 9PL, U.K..

E-mail address: Toby.Stafford@manchester.ac.uk 